\title{
Finite time extinction for the 1D stochastic porous medium equation with transport noise
}

\author{
Sebastian Hensel ${ }^{1}$
}

Received: 7 February 2020 / Revised: 11 November 2020 / Accepted: 8 February 2021 /

Published online: 21 March 2021

(C) The Author(s) 2021

\begin{abstract}
We establish finite time extinction with probability one for weak solutions of the Cauchy-Dirichlet problem for the 1D stochastic porous medium equation with Stratonovich transport noise and compactly supported smooth initial datum. Heuristically, this is expected to hold because Brownian motion has average spread rate $O\left(t^{\frac{1}{2}}\right)$ whereas the support of solutions to the deterministic PME grows only with rate $O\left(t^{\frac{1}{m+1}}\right)$. The rigorous proof relies on a contraction principle up to time-dependent shift for Wong-Zakai type approximations, the transformation to a deterministic PME with two copies of a Brownian path as the lateral boundary, and techniques from the theory of viscosity solutions.
\end{abstract}

Keywords Stochastic porous medium equation - Finite time extinction · Wong-Zakai approximation · Contraction principle

Mathematics Subject Classification Primary 60H15 · 35R60; Secondary 35R35 . 35R37

\section{Introduction}

We study the stochastic porous medium equation with Stratonovich transport noise

\begin{abstract}
This project has received funding from the European Union's Horizon 2020 research and innovation programme under the Marie Skłodowska-Curie Grant Agreement No. 665385 . I am very grateful to M. Gerencsér and J. Maas for proposing this problem as well as helpful discussions. Special thanks go to F. Cornalba for suggesting the additional $\kappa$-truncation in Proposition 5. I am also indebted to an anonymous referee for pointing out a gap in a previous version of the proof of Lemma 9 (concerning the treatment of the noise term). The issue is resolved in this version.
\end{abstract}

Sebastian Hensel sebastian.hensel@ist.ac.at

1 Institute of Science and Technology Austria (IST Austria), Am Campus 1, 3400 Klosterneuburg, Austria 


$$
\mathrm{d} u=\Delta u^{m} \mathrm{~d} t+v \nabla u \circ \mathrm{d} B_{t}
$$

in the space-time cylinder $Q=D \times(0, T)$ for some bounded convex domain $D \subset \mathbf{R}^{d}$ with $C^{2}$ boundary $\partial D$ and time horizon $T \in(0, \infty]$. Here $u \geq 0$ denotes the so-called density variable, $B$ is a $d$-dimensional standard Brownian motion and $m>1$ as well as $v>0$ are parameters. Moreover, the Stratonovich differential $v \nabla u \circ \mathrm{d} B_{t}$ is an abbreviation for the expression $\frac{1}{2} v^{2} \Delta u \mathrm{~d} t+v \nabla u \cdot \mathrm{d} B_{t}$.

We are in particular interested in studying the corresponding Cauchy-Dirichlet problem, i.e., we supplement the stochastic PDE (1) with

$$
\begin{aligned}
& u(x, 0)=u_{0}(x), \quad x \in \bar{D}, \\
& u(x, t)=0, \quad(x, t) \in \partial D \times(0, T] .
\end{aligned}
$$

The initial condition is given by a non-negative smooth function $u_{0} \in C_{\mathrm{cpt}}^{\infty}(D)$ with compact support in $D$. In particular, (2) and (3) are compatible to any order.

The main result of the present work is that weak solutions (in the precise sense of Definition 1 below) to the 1D Cauchy-Dirichlet problem of the SPME (1)-(3) become extinct in finite time on a set of full probability. For a mathematically precise statement we refer the reader to Theorem 3 below. To the best of the author's knowledge, this is the first result establishing finite time extinction in the slow diffusion regime $m>1$ for the stochastic porous medium equation. In contrast, finite time extinction in the fast diffusion regime $0<m<1$ is well known in several cases. For instance, Barbu, Da Prato and Röckner establish finite time extinction for porous medium type models with multiplicative noise in the works [2-4]. Gess treats the case of stochastic sign fast diffusion equations in [13]. In a very recent work of Turra [18], finite time extinction in the fast diffusion regime is established for transport noise. The slow diffusion case with transport noise, however, is left open.

The underlying idea of the present work is to exploit that the support of solutions to the deterministic porous medium equation grows only with rate $O\left(t^{\frac{1}{m+1}}\right)$ whereas Brownian motion spreads on average with a faster rate $O\left(t^{\frac{1}{2}}\right)$. Hence one may expect that the effect of the random advection term in (1) eventually dominates the non-linear diffusion term, thus leading to finite time extinction on a set of full probability. Let us briefly mention the three main ingredients which provide a rigorous justification for this heuristic (for a more detailed and mathematical account on the strategy, we refer the reader to Sect. 3). Along the way we provide connections to the-for the purposes of this work - relevant parts of the by now extensive literature for the stochastic porous medium equation.

In a first step, we recover the unique weak solution (in the sense of Definition 1 below) to the Cauchy-Dirichlet problem of the SPME (1)-(3) by means of a suitable Wong-Zakai approximation. We then perform, on the level of the approximate solutions, a simple stochastic flow transformation yielding smooth solutions to a deterministic porous medium equation, but now with two copies of a mollified Brownian path as the lateral boundary. In principle, we would then like to remove the mollification parameter to formally obtain a subsolution to the deterministic PME with two copies of a Brownian path as the lateral boundary. We may view this object-again 
at least on a formal level—as a subsolution to the full space porous medium equation by trivially extending it outside of the domain. We finally put a Barenblatt profile above the solution at the initial time. It follows by the comparison principle that finite time extinction happens once the domain with two copies of a Brownian path as the lateral boundary is pushed outside the support of such a Barenblatt solution. Since the support of the Barenblatt solution has finite speed of propagation of order $O\left(t^{\frac{1}{m+1}}\right)$, this actually occurs with probability one.

However, two technical issues being linked to each other arise in the above strategy. The first is concerned with the proposed limit passage after the stochastic flow transformation. The problem is that the domains on which the approximate and transformed solutions are supported are not monotonically ordered as one sends the mollification scale to zero. This fact together with the desire to make use of the comparison principle motivated us to perform this limit passage in the framework of viscosity theory by means of the technique of semi-relaxed limits. This allows us to rigorously construct a maximal subsolution for the deterministic porous medium equation with two copies of a Brownian path as the lateral boundary (to be understood in the precise sense of Definition 14 below). To the best of the author's knowledge, the present work seems to be the first instance to make use of viscosity theory to study the Dirichlet problem for the stochastic porous medium equation after stochastic flow transformation.

A notion of viscosity solution for the full space deterministic porous medium equation has been developed by Caffarelli and Vázquez [5] (see also the work of Vázquez and Brändle [20]). However, as a consequence of the degeneracy of the porous medium equation the usual notion of supersolutions from [6] had to be adapted. But since we only need to talk about subsolutions for our purposes, this is actually of no concern for us.

However, by taking a semi-relaxed limit it is by no means clear anymore how this maximal subsolution and the transformed unique weak solution to (1)-(3) relate to each other. In order to close the argument, we need to make sure that the unique weak solution of the original problem (1)-(3) is dominated after stochastic flow transformation by the constructed maximal subsolution in a suitable sense. It turns out that we can justify this once we provided convergence of the Wong-Zakai approximations in a strong enough topology like $L^{1}$. Let us mention at this point the recent work of Fehrman and Gess [11], who prove rough path well-posedness for nonlinear gradient type noise using a kinetic formulation which in particular implies Wong-Zakai type results, but with the equation being posed on the torus.

There has been recently a lot of focus on providing well-posedness for stochastic porous medium equations in an $L^{1}$ framework. The corresponding notion of solution is the one of entropy solutions first put forward in the work of Dareiotis, Gerencsér and Gess [7], and the theory is based on a quantitative $L^{1}$ contraction principle for such solutions. In the work [7], the authors consider the case of nonlinear multiplicative noise with periodic boundary conditions. The subsequent work of Dareiotis and Gess [9] treats the regime of nonlinear conservative gradient noise (again with periodic boundary conditions), whereas the recent work [10] by Dareiotis, Gess and Tsatsoulis provides the corresponding theory of [7] for the Dirichlet problem on smooth domains. 
Following ideas developed in [7], we roughly speaking aim to establish $L^{1}$ convergence of the sequence of Wong-Zakai approximations. However, as we are concerned in this work with the Cauchy-Dirichlet problem for the SPME with transport noise, the actual implementation differs in some aspects to the above mentioned works. Most importantly, since two different approximate solutions are advected with different speeds (as a consequence of the two associated different mollification scales) it is natural to introduce a time-dependent, random shift function and to compare the Wong-Zakai approximations only after shifting. We then essentially establish quantitative $L^{1}$ contraction for this sequence of shifted densities, see Proposition 5 below for the mathematically precise formulation. This result may be of independent interest.

\section{Notation}

Throughout the paper, we fix a filtered probability space $(\Omega, \mathcal{F}, \mathbf{F}, \mathbf{P})$ with filtration $\mathbf{F}=\left(\mathcal{F}_{t}\right)_{t \in[0, \infty)}$ which is subject to the usual conditions, i.e., the filtration $\mathbf{F}$ is right-continuous and $\mathcal{F}_{0}$ is $\mathbf{P}$-complete. By $B=\left(B_{t}\right)_{t \in[0, \infty)}$ we denote a standard $d$-dimensional F-Brownian motion. For a given time horizon $T \in(0, \infty]$ we let $\mathcal{P}_{T}$ be the predictable $\sigma$-field on $\Omega_{T}:=\Omega \times[0, T]$.

For two maps $f$ and $g$, we write $f \circ g$ to denote the composition of $f$ with $g$. We slightly abuse notation here since we already use the symbol $\circ$ for Stratonovich integration. However, it will always be clear from the context in which sense it is to be interpreted.

Let $D \subset \mathbf{R}^{d}$ be an open domain. (In the one-dimensional setting, we will instead use the notation $I \subset \mathbf{R}$ for a bounded open interval.) The space of all smooth functions with compact support in $D$ is denoted by $C_{\mathrm{cpt}}^{\infty}(D)$, whereas $C^{0}(\bar{D})$ represents the space of all continuous functions on the closure $\bar{D}$ with bounded supremum norm. The space $H_{0}^{1}(D)$ is the usual space of Sobolev functions with zero trace on the boundary. Its topological dual space will be denoted by $H^{-1}$. The space of $H^{-1}$ valued, continuous and $\mathbf{F}$-adapted stochastic processes intersected with $L^{q}\left(\Omega_{T}, \mathcal{P}_{T} ; L^{q}(D)\right)$ is denoted by $\mathbf{H}_{q}^{-1}(D)$. The space of $L^{2}(D)$ valued, continuous and $\mathbf{F}$-adapted stochastic processes intersected with the space $L^{2}\left(\Omega_{T}, \mathcal{P}_{T} ; H_{0}^{1}(D)\right)$ ) (resp. the space $\left.L^{2}\left(\Omega_{T}, \mathcal{P}_{T} ; H^{1}(D)\right)\right)$ is $\mathbf{L}_{0}^{2}(D)$ (resp. $\left.\mathbf{L}^{2}(D)\right)$. If we want to specify the target values for a function space, we will include this in the notation. E.g., $C_{\mathrm{cpt}}^{\infty}(D ;[0, \infty))$ is the space of all non-negative smooth functions with compact support in $D$. The space of functions of bounded variation is denoted by $B V$.

The Lebesgue measure on $\mathbf{R}^{d}$ is denoted by $\mathcal{L}^{d}$, the $s$-dimensional Hausdorff measure by $\mathcal{H}^{s}$. We write $\chi_{A}$ for the indicator function of a measurable set $A$ (with respect to any measure space). For two numbers $a, b \in \mathbf{R}$ we abbreviate $a \vee b$ for their maximum respectively $a \wedge b$ for their minimum. Finally, we define $a_{+}:=a \vee 0$ and $\operatorname{sign}_{+}(a):=\chi_{(0, \infty)}(a)$ for $a \in \mathbf{R}$. 


\section{Main result}

The main result of the present work establishes that weak solutions of the onedimensional stochastic porous medium equation with Stratonovich transport noise (1)-(3) almost surely have the finite time extinction property. Before we provide the precise statement, let us introduce the notion of weak solutions.

Definition 1 Let $D \subset \mathbf{R}^{d}$ be a bounded domain with $C^{2}$ boundary, $T^{*} \in(0, \infty]$ be a time horizon as well as $u_{0} \in C_{\mathrm{cpt}}^{\infty}(D ;[0, \infty))$ be an initial density. A non-negative stochastic process $u \in \mathbf{H}_{m+1}^{-1}(D)$ is called a weak solution to the Cauchy-Dirichlet problem (1)-(3) of the stochastic porous medium equation (SPME) with initial density $u_{0}$ on the space time cylinder $Q=D \times\left(0, T^{*}\right)$ if for all $\phi \in C_{\mathrm{cpt}}^{\infty}(D)$ with probability one it holds

$$
\begin{aligned}
& \int_{D} u(x, T) \phi(x) \mathrm{d} x-\int_{D} u_{0}(x) \phi(x) \mathrm{d} x \\
& \quad=\int_{0}^{T} \int_{D}\left(u^{m}(x, t)+\frac{1}{2} v^{2} u(x, t)\right) \Delta \phi(x) \mathrm{d} x \mathrm{~d} t-\int_{0}^{T} \int_{D} \nu u(x, t) \nabla \phi(x) \mathrm{d} x \mathrm{~d} B_{t}
\end{aligned}
$$

for all $T \in\left(0, T^{*}\right)$.

Remark 2 Existence and uniqueness of weak solutions in the sense of Definition 1 was established, for instance, in [8, Theorem 3.3]. In fact, the authors consider a much more general class of degenerate quasilinear stochastic PDE, allowing for more general initial conditions and, in particular, for signed solutions.

Being equipped with the notion of weak solutions we may now formulate the main result of the present work, which is finite time extinction with probability one for weak solutions of the 1D stochastic porous medium equation with Stratonovich transport noise.

Theorem 3 Let I $\subset \mathbf{R}$ be a bounded open interval, and let $u \in \mathbf{H}_{m+1}^{-1}(I)$ be the unique weak solution to the SPME (1)-(3) with initial density $u_{0} \in C_{\mathrm{cpt}}^{\infty}(I ;[0, \infty))$ on the space-time cylinder $I \times(0, \infty)$ in the sense of Definition 1. Define a stopping time by means of

$$
T_{\text {extinct }}:=\inf \{T \geq 0: u(\cdot, T)=0 \text { almost everywhere in } I\} \text {. }
$$

Then there exists a constant $M=M\left(u_{0}\right)>0$ depending only on the initial density such that

$$
\mathbf{P}\left(T_{\text {extinct }} \leq T\right) \geq \mathbf{P}\left(\inf \left\{t \geq 0:\left|B_{t}\right| \geq \mathcal{L}^{1}(I)+M t^{\frac{1}{m+1}}\right\} \leq T\right)
$$

for all $T \in(0, \infty)$. Moreover, for $\hat{T}_{\text {extinct }}:=\inf \left\{T \geq 0:\left|B_{T}\right| \geq \mathcal{L}^{1}(I)+M T^{\frac{1}{m+1}}\right\}$ it holds $\mathbf{P}\left(T_{\text {extinct }}<\infty\right)=\mathbf{P}\left(\hat{T}_{\text {extinct }}<\infty\right)=1$. Finally, for all $T \in(0, \infty)$ we have almost surely on $\left\{T \geq \hat{T}_{\text {extinct }}\right\}$ that $u(\cdot, T)=0$ is satisfied almost everywhere in $I$. 


\section{Outline of the strategy}

Let us comment on the strategy for the proof of the main result, Theorem 3. In a nutshell the argument works as follows. By stochastic flow transformation, which in our case of pure transport noise is of particularly easy form, we may consider first the transformed densities

$$
v(x, t):=u\left(x-v B_{t}, t\right), \quad(x, t) \in \bigcup_{t \in(0, \infty)}\left(v B_{t}+I\right) \times\{t\} .
$$

Formally, the transformed density $v$ is then subject to the deterministic porous medium equation

$$
\partial_{t} v=\partial_{x x} v^{m} \text { in } \bigcup_{t \in(0, \infty)}\left(v B_{t}+I\right) \times\{t\},
$$

with initial density $v(\cdot, 0)=u_{0}$ and Dirichlet boundary conditions on the lateral boundary $\bigcup_{t \in[0, \infty)}\left(v B_{t}+\partial I\right) \times\{t\}$. Extending $v$ trivially to the entire space-time domain $\mathbf{R} \times(0, \infty)$, and denoting this extension by $\bar{v}$, we obtain, at least formally, a subsolution to the Cauchy problem of the deterministic porous medium equation $\partial_{t} \bar{v}=\partial_{x x} \bar{v}^{m}$ with initial density $\bar{v}(\cdot, 0)=u_{0} \geq 0$.

Now, we may choose a Barenblatt profile $\overline{\mathcal{B}}$ with free boundary $\partial I$ at $t=0$ and which strictly dominates the initial density, i.e., $\{\mathcal{B}(\cdot, 0)=0\}=\partial I$ and $u_{0}<\mathcal{B}(\cdot, 0)$ on $I$. By comparison it follows $\bar{v} \leq \mathcal{B}$ on $\mathbf{R} \times(0, \infty)$. Since the support of the Barenblatt solution has finite speed of propagation of order $O\left(T^{\frac{1}{m+1}}\right)$ (with the implicit constant only depending on the initial density), we deduce the validity of (6). However, since Brownian motion has average spread rate of order $O\left(T^{\frac{1}{2}}\right)$ and we assume that $m>1$, this entails that finite time extinction happens with probability one: $\mathbf{P}\left(T_{\text {extinct }}<\infty\right)=$ 1 .

To make this argument rigorous, we rely on a suitable approximation procedure. To this end, we first consider the $d$-dimensional setting and fix a finite time horizon $T^{*} \in(0, \infty)$. Moreover, choose $\rho \in C_{\mathrm{cpt}}^{\infty}((0,1) ;[0, \infty))$ with $\int_{\mathbf{R}} \rho(r) \mathrm{d} r=1$ and define $\rho_{\varepsilon}:=\frac{1}{\varepsilon} \rho(\dot{\bar{\varepsilon}})$ for $\varepsilon>0$. We then introduce the smooth approximations $B^{\varepsilon}=$ $\left(B_{t}^{\varepsilon}\right)_{t \in[0, \infty)}$ to Brownian motion defined via

$$
B_{t}^{\varepsilon}:=\int_{0}^{\infty} \rho_{\varepsilon}(t-s) B_{s} \mathrm{~d} s
$$

Since the mollifier $\rho$ is supported on positive times, we note that $B^{\varepsilon}$ is $\mathbf{F}$-adapted. We also have almost surely that

$$
\sup _{\varepsilon>0}\left\|B^{\varepsilon}\right\|_{C^{0}\left(\left[0, T^{*}\right]\right)}<\infty
$$


as well as

$$
B^{\varepsilon} \rightarrow B \text { uniformly on }\left[0, T^{*}\right] \text { as } \varepsilon \rightarrow 0 \text {. }
$$

Fix $\alpha \in\left(\frac{1}{3}, \frac{1}{2}\right)$. For purely technical reasons, we make also use of the classical fact that there is a square integrable random variable $\mathcal{C}_{\alpha}$ such that with probability one

$$
\left|B_{t}-B_{s}\right| \leq \mathcal{C}_{\alpha}|t-s|^{\alpha} \text { for all } s, t \in\left[0, T^{*}\right]
$$

In particular, for each integer $M \geq 1$ and each $\delta>0$ we can find an $\varepsilon^{\prime}=\varepsilon^{\prime}(M, \delta)>0$ such that for all $\varepsilon \leq \varepsilon^{\prime}$ it holds

$$
\sup _{t \in\left[0, T^{*}\right]}\left|B_{t}-B_{t}^{\varepsilon}\right| \leq \delta \text { almost surely on }\left\{\mathcal{C}_{\alpha} \leq M\right\}
$$

We then proceed by considering the inhomogeneous Cauchy-Dirichlet problem

$$
\begin{aligned}
\mathrm{d} u_{\varepsilon} & =\Delta u_{\varepsilon}^{m} \mathrm{~d} t+v \nabla u_{\varepsilon} \cdot \mathrm{d} B_{t}^{\varepsilon}, & (x, t) & \in D \times\left(0, T^{*}\right), \\
u_{\varepsilon}(x, 0) & =u_{0}(x)+\varepsilon, & x & \in \bar{D}, \\
u_{\varepsilon}(x, t) & =\varepsilon, & (x, t) & \in \partial D \times\left(0, T^{*}\right] .
\end{aligned}
$$

Note that the regularization not only comes from the Wong-Zakai approximation $\nabla u_{\varepsilon} \cdot \mathrm{d} B_{t}^{\varepsilon}=\left(\frac{\mathrm{d}}{\mathrm{d} t} B_{t}^{\varepsilon} \cdot \nabla\right) u_{\varepsilon} \mathrm{d} t$, but also from choosing strictly positive initial and boundary data. In this way we circumvent the degeneracy at $u=0$ of the porous medium operator at the level of the approximations, i.e., we can solve the problem (12)-(14) in a classical and pathwise sense such that the maximum principle applies. Proofs will be provided in the subsequent Sect. 4.

Lemma 4 For each $\varepsilon>0$, there exist $u_{\varepsilon} \in \varepsilon+\mathbf{L}_{0}^{2}(D)$ such that the following holds true on a set with probability one (e.g., so that (8) holds):

For all $\varepsilon>0$ the map $u_{\varepsilon}$ is the unique classical solution of the Cauchy-Dirichlet problem (12)-(14) in the sense that $u_{\varepsilon} \in C_{x, t}^{2,1}\left(\bar{D} \times\left[0, T^{*}\right]\right) \cap C^{\infty}\left(D \times\left(0, T^{*}\right)\right)$ and the equations (12)-(14) are satisfied pointwise everywhere. From the maximum principle, we have the bounds

$$
\varepsilon \leq u_{\varepsilon}(x, t) \leq \varepsilon+\left\|u_{0}\right\|_{L^{\infty}(D)}
$$

for all $\varepsilon>0$ and all $(x, t) \in \bar{D} \times\left[0, T^{*}\right]$. There is a constant $C=C\left(T^{*}, v, u_{0}\right)>0$ and some $\beta>0$ such that the a priori estimates

$$
\begin{gathered}
\sup _{T \in\left[0, T^{*}\right]} \int_{D} \frac{1}{2}\left|u_{\varepsilon}\right|^{2}(T) \mathrm{d} x+\int_{0}^{T^{*}} \int_{D} m u_{\varepsilon}^{m-1}\left|\nabla u_{\varepsilon}\right|^{2} \mathrm{~d} x \mathrm{~d} t \leq \int_{D} \frac{1}{2}\left|u_{\varepsilon}(0)\right|^{2} \mathrm{~d} x, \\
\sup _{T \in\left[0, T^{*}\right]} \mathbf{E} \int_{D} \frac{T}{2}\left|\nabla u_{\varepsilon}^{m}\right|^{2}(T) \mathrm{d} x+\mathbf{E} \int_{0}^{T^{*}} \int_{D} \frac{t}{2} m u_{\varepsilon}^{m-1}\left|\partial_{t} u_{\varepsilon}\right|^{2} \mathrm{~d} x \mathrm{~d} t \leq \varepsilon^{-\beta} C,
\end{gathered}
$$




$$
\mathbf{E} \int_{0}^{T^{*}} \int_{D} t\left|\Delta u_{\varepsilon}^{m}\right|^{2} \mathrm{~d} x \mathrm{~d} t \leq \varepsilon^{-\beta} C
$$

\section{hold true almost surely for all $\varepsilon>0$.}

A key ingredient for the rigorous justification of the comparison argument outlined at the beginning of this section is that the Wong-Zakai type approximations $u_{\varepsilon}$ from Lemma 4 recover the unique weak solution $u$ to the Cauchy-Dirichlet problem (1)(3) in a certain pointwise sense. We obtain this by establishing a sort of contraction principle for the sequence of $u_{\varepsilon}$ by means of Kružkov's device of variable doubling [14]. To this end, the proof loosely follows the strategy of Dareiotis, Gerencsér and Gess [7] or Dareiotis and Gess [9] who study entropy solutions for stochastic porous medium type equations posed on the torus. The Cauchy-Dirichlet problem in the framework of entropy solutions was recently studied in the work of Dareiotis, Gess and Tsatsoulis [10]. However, they do not consider the case of gradient type noise. Let us also mention the recent work of Fehrman and Gess [11], who prove rough path well-posedness for nonlinear gradient type noise using a kinetic formulation which in particular implies Wong-Zakai type results, but with the equation being posed on the torus..

As we are concerned in this work with the Cauchy-Dirichlet problem for the SPME with transport noise, and roughly speaking aim to establish $L^{1}$ convergence for the Wong-Zakai type approximations $u_{\varepsilon}$, the actual implementation of the doubling of variables technique differs in some aspects to the above mentioned works. First, since two different solutions $u_{\varepsilon}$ and $u_{\hat{\varepsilon}}$ are advected at different speeds, it seems to be natural to introduce for our purpose a (time-dependent, random) shift function and to compare the solutions only after shifting, i.e., we are led to study $L^{1}$ convergence for the sequence

$$
u_{\varepsilon}^{\leftarrow}(x, t):=u_{\varepsilon}\left(x+v\left(B_{t}-B_{t}^{\varepsilon}\right), t\right)
$$

Second, since the introduction of this shift in turn changes the domain on which the equation for $u_{\varepsilon}^{\leftarrow}$ is posed it is necessary to create a boundary layer in order to apply the doubling of variables method up to the boundary. This is done by means of an additional truncation as follows.

Let $\kappa>0$ and $\delta \in\left(0, \frac{\kappa}{2}\right)$ be fixed. We then choose a smooth and convex map $\zeta: \mathbf{R} \rightarrow[0, \infty)$ such that $\zeta(r)=0$ for $r \leq 0, \zeta(r)=r-1$ for $r \geq 2$ and $\zeta(r) \leq r \vee 0$ for all $r \in \mathbf{R}$. Define $\zeta_{\delta}(r):=\delta \zeta\left(\frac{r}{\delta}\right)$. There is a constant $C>0$ independent of $\delta$ such that

$$
\begin{aligned}
& \sup _{r \in \mathbf{R}}\left|\left(\zeta_{\delta}\right)^{\prime}(r)\right|+|r|\left(\zeta_{\delta}\right)^{\prime \prime}(r) \leq C, \\
& \left(\zeta_{\delta}\right)^{\prime}(r) \rightarrow \operatorname{sign}_{+}(r) \text { and } \zeta_{\delta}(r) \nearrow r_{+}:=\max \{r, 0\} \text { as } \delta \rightarrow 0, \\
& \left|\zeta_{\delta}(r)-r_{+}\right| \leq C \delta \text { for all } r \in \mathbf{R}, \text { and } \zeta_{\delta}(r)=r-\delta \text { for all } r \geq 2 \delta .
\end{aligned}
$$

Let $\zeta_{\kappa}^{\delta}(r):=\kappa+\zeta_{\delta}(r-\kappa)$. The idea then is to study basically $L^{1}$ contraction for the sequence $\zeta_{\kappa}^{\varepsilon} \circ u_{\varepsilon}^{\leftarrow}$ for $\varepsilon \leq \frac{\kappa}{2}$. Note that for each time slice $t \in\left[0, T^{*}\right]$ the 
function $\zeta_{\kappa}^{\varepsilon} \circ u_{\varepsilon}^{\leftarrow}(\cdot, t)$ is $C^{\infty}$ in the open domain $-v\left(B_{t}-B_{t}^{\varepsilon}\right)+D$, and for $\varepsilon \leq \frac{\kappa}{2}$ it is actually constant in a neighborhood of $-v\left(B_{t}-B_{t}^{\varepsilon}\right)+\partial D$. Extending in each time slice $u_{\varepsilon}$ to $\varepsilon$ outside of the domain $D$, and denoting this extension again by $u_{\varepsilon}$, we see that $\zeta_{\kappa}^{\varepsilon} \circ u_{\varepsilon}^{\leftarrow}(\cdot, t)$ is $C^{\infty}$ even on the whole space $\mathbf{R}^{d}$. In summary, by introducing a boundary layer as above there is no jump of the Neumann data for $\zeta_{\kappa}^{\varepsilon} \circ u_{\varepsilon}^{\leftarrow}$ across the boundary $-v\left(B_{t}-B_{t}^{\varepsilon}\right)+\partial D$. This turns out to be absolutely essential in order to apply the doubling of variables technique up to the boundary.

Proposition 5 (Contraction principle up to time-dependent shift for truncated WongZakai type approximations) For each $\varepsilon>0$ let $u_{\varepsilon}$ denote the unique classical solution to (12)-(14) in the sense of Lemma 4 . We extend $u_{\varepsilon}$ to a function defined on $\mathbf{R}^{d} \times\left[0, T^{*}\right]$ by setting it equal to $\varepsilon$ outside of $D \times\left[0, T^{*}\right]$. Denoting this extension again by $u_{\varepsilon}$ we then define for all $\varepsilon>0$ the shifted densities

$$
u_{\varepsilon}^{\leftarrow}(x, t):=u_{\varepsilon}\left(x+v\left(B_{t}-B_{t}^{\varepsilon}\right), t\right), \quad(x, t) \in \mathbf{R}^{d} \times\left[0, T^{*}\right] .
$$

There exists a constant $\bar{C}>0$ and an exponent $\vartheta>0$ such that for all truncation levels $\kappa \in\left(0, T^{*} \wedge 1\right)$ and all compact sets $K \subset D$, there exists a small constant $\varepsilon_{0}=\varepsilon_{0}(\kappa, K)$ such that for all $\varepsilon, \hat{\varepsilon} \leq \varepsilon_{0}$ it holds

$$
\begin{aligned}
& \sup _{T \in\left[\kappa, T^{*}\right]} \mathbf{E} \int_{K}\left|\kappa \vee u_{\varepsilon}^{\leftarrow}(x, T)-\kappa \vee u_{\hat{\varepsilon}}^{\leftarrow}(x, T)\right| \mathrm{d} x \\
& \leq \bar{C}(\varepsilon \vee \hat{\varepsilon})^{\vartheta}+\bar{C} \kappa+\mathbf{E} \int_{D}\left|\kappa \vee\left(u_{0}(x)+\varepsilon\right)-\kappa \vee\left(u_{0}(x)+\hat{\varepsilon}\right)\right| \mathrm{d} x
\end{aligned}
$$

We may lift this quantitative stability estimate for the truncated and shifted densities $\kappa \vee u_{\varepsilon}^{\leftarrow}$ to qualitative $L^{1}$ convergence of the shifted densities $u_{\varepsilon}^{\leftarrow}$.

Corollary 6 Let the assumptions and notation of Proposition 5 be in place. Then the sequence of shifted densities $u_{\varepsilon}^{\leftarrow}$ is Cauchy in $C\left(\left[\tau, T^{*}\right] ; L^{1}\left(\Omega \times D, \mathbf{P} \otimes \mathcal{L}^{d}\right)\right)$ for all positive times $\tau \in\left(0, T^{*}\right)$. The sequence of shifted densities is moreover Cauchy in $L^{1}\left(\left[0, T^{*}\right] ; L^{1}\left(\Omega \times D, \mathbf{P} \otimes \mathcal{L}^{d}\right)\right)$. Let $u \in L^{1}\left(\left[0, T^{*}\right] ; L^{1}\left(\Omega \times D, \mathbf{P} \otimes \mathcal{L}^{d}\right)\right)$ denote the corresponding limit in this space. Then, $u$ is also the weak limit of the Wong-Zakai type approximations $u_{\varepsilon}$ from Lemma 4 in the space $L^{m+1}\left(\Omega_{T^{*}}, \mathcal{P}_{T^{*}} ; L^{m+1}(D)\right)$

The final issue concerning the Wong-Zakai type approximations $u_{\varepsilon}$ from Lemma 4 is the identification of the limit object $u$ in Corollary 6 as the unique weak solution to the Cauchy-Dirichlet problem (1)-(3) of the stochastic porous medium equation.

Proposition 7 Let $u \in L^{m+1}\left(\Omega_{T^{*}}, \mathcal{P}_{T^{*}} ; L^{m+1}(D)\right)$ be the limit of the Wong-Zakai approximations $u_{\varepsilon}$ in the sense of Corollary 6 . Then it holds that $u \in \mathbf{H}_{m+1}^{-1}(D)$, and $u$ is the unique weak solution to the Cauchy-Dirichlet problem (1)-(3) with initial density $u_{0} \in C_{\mathrm{cpt}}^{\infty}(D ;[0, \infty))$ in the sense of Definition 1. Moreover, u satisfies the bounds $0 \leq u \leq\left\|u_{0}\right\|_{L^{\infty}(D)}$.

From now on we will restrict ourselves to the one-dimensional setting $d=1$. We still have to make rigorous the outlined comparison argument. The key ingredient 
for this will be provided in Sect. 5.1. It consists of the construction of a maximal subsolution $\bar{p}_{\max }$ (in the sense of viscosity theory [6], precise definitions will follow in Sect. 5.1) for the Cauchy-Dirichlet problem of the porous medium equation after stochastic flow transformation

$$
\begin{aligned}
\partial_{t} \bar{p} & =(m-1) \bar{p} \partial_{x x} \bar{p}+\left|\partial_{x} \bar{p}\right|^{2}, & (x, t) & \in \bigcup_{t \in\left(0, T^{*}\right)}\left(v B_{t}+I\right) \times\{t\}, \\
\bar{p}(x, 0) & =\bar{p}_{0}(x), & x & \in \bar{I}, \\
\bar{p}(x, t) & =0, & (x, t) & \in \bigcup_{t \in\left(0, T^{*}\right]}\left(v B_{t}+\partial I\right) \times\{t\} .
\end{aligned}
$$

The pressure variable $p$ is obtained from the density variable $u$ via the transformation $g(u)$ with $g:[0, \infty) \rightarrow[0, \infty)$ given by $g(r):=\frac{m}{m-1} r^{m-1}$. To the best of the author's knowledge, the present work seems to be the first instance to make use of the pressure formulation to study the stochastic porous medium equation after stochastic flow transformation in the setting of viscosity theory.

The main difficulty for solving (24)-(26) comes from the fact that the lateral boundary consists of two translates of a Brownian trajectory. To overcome the lack of regularity of the lateral boundary, we first consider the approximate initial-boundary value problem (see Lemma 15)

$$
\begin{aligned}
\partial_{t} \bar{p}_{\varepsilon} & =(m-1) \bar{p}_{\varepsilon} \partial_{x x} \bar{p}_{\varepsilon}+\left|\partial_{x} \bar{p}_{\varepsilon}\right|^{2}, & (x, t) & \in \bigcup_{t \in\left(0, T^{*}\right)}\left(v B_{t}^{\varepsilon}+I\right) \times\{t\}, \\
\bar{p}_{\varepsilon}(x, 0) & =\bar{p}_{0, \varepsilon}(x), & x & \in \bar{I}, \\
\bar{p}_{\varepsilon}(x, t) & =\frac{m}{m-1} \varepsilon^{m-1}, & (x, t) & \in \bigcup_{t \in\left(0, T^{*}\right]}\left(v B_{t}^{\varepsilon}+\partial I\right) \times\{t\},
\end{aligned}
$$

and then pass to the limit $\varepsilon \rightarrow 0$ by means of the technique of semi-relaxed limits, see for instance [6, Section 6]. In this way we obtain a maximal subsolution to the problem (24)-(26) in the sense of viscosity theory [6], see Proposition 16. The main motivation for working in the framework of viscosity theory is the non-monotonicity of the underlying space-time domains $\bigcup_{t \in\left(0, T^{*}\right)}\left(\nu B_{t}^{\varepsilon}+I\right) \times\{t\}$ as $\varepsilon \rightarrow 0$ which necessitates the usage of a relaxed limit. In this way, however, the interpretation of the lateral boundary condition (26) in a strong sense is lost in the limit. It is well-known that boundary regularity for solutions to stochastic PDEs with gradient type noise and given Dirichlet data proves to be a delicate issue. This already shows up in the linear case, see, for instance, the works by Krylov [15] and [16]. For a recent work in the semilinear regime, we refer the reader to [12]. On the other side, "continuity up to the lateral boundary" is inessential for our purposes and anyway not expected, if at all, to be obtained by the methods in this work.

Note that the maximal subsolution $\bar{p}_{\max }$ of course depends on the realization of Brownian motion, and is therefore random. However, since the construction of $\bar{p}_{\max }$ is ultimately a purely deterministic consequence of the probabilistic facts (8)-(11), we obtain the maximal subsolution in a pathwise sense on a set of full probability. 
The proof of Theorem 3, which is the content of Sect. 5.5, then roughly speaking proceeds as follows. Denoting by $p$ the map which we obtain from the unique weak solution $u$ of (1)-(3) in the sense of Definition 1 by first applying a stochastic flow transformation and then a density-to-pressure transformation, we have the estimate $p \leq \bar{p}_{\max }$, see Proposition 17 . This bound is essentially a combination of the following facts:

(i) The solution to (27)-(29) may in fact be obtained from the Wong-Zakai type approximations $u_{\varepsilon}$ of (12)-(14) by first applying a stochastic flow transformation and then a density-to-pressure transformation.

(ii) The Wong-Zakai approximations $u_{\varepsilon}$ (or more precisely, their shifted counterparts (22)) converge on each positive time slice in $L^{1}$ to the unique weak solution of the Cauchy-Dirichlet problem (1)-(3), see Corollary 6.

(iii) The maximal subsolution to (24)-(26) dominates the upper semi-relaxed limit (with respect to parabolic space-time cylinders) of the transformed $u_{\varepsilon}$. However, taking a semi-relaxed limit allows to compare with the transformed density $u$ by means of the previous two items.

Finally, we compare the maximal subsolution $\bar{p}_{\text {max }}$ to a Barenblatt profile (written in the pressure variable) as outlined in the heuristic argument. We make use of the comparison principle in the framework of viscosity solutions for the deterministic porous medium equation as developed by Caffarelli and Vázquez [5] resp. Vázquez and Brändle [20]. The remaining argument after comparing with the Barenblatt profile, in particular the derivation of (6), then works as already sketched before.

\section{Recovering weak solutions by Wong-Zakai type approximations}

\subsection{Proof of Lemma 4 (Wong-Zakai type approximation)}

We make use of a usual trick of avoiding the degeneracy of the porous medium operator, see for instance [19, Proof of Theorem 5.5]. Let $\varepsilon>0$ be fixed and choose a bounded smooth function $a_{\varepsilon}: \mathbf{R} \rightarrow\left[m\left(\frac{\varepsilon}{2}\right)^{m-1}, \infty\right)$ such that it holds $a_{\varepsilon}(r)=m r^{m-1}$ for all $r \in\left[\varepsilon, \varepsilon+\left\|u_{0}\right\|_{L^{\infty}(D)}\right]$. By the choice of $a_{\varepsilon}$ and since $B_{t}^{\varepsilon}$ as defined in (7) is smooth on $\left[0, T^{*}\right]$ almost surely, we can make use of standard quasilinear theory [17] to solve the PDE

$$
\partial_{t} u_{\varepsilon}=\nabla \cdot\left(a_{\varepsilon}\left(u_{\varepsilon}\right) \nabla u_{\varepsilon}\right)+v\left(\frac{\mathrm{d}}{\mathrm{d} t} B_{t}^{\varepsilon} \cdot \nabla\right) u_{\varepsilon} \quad \text { on } \bar{D} \times\left[0, T^{*}\right]
$$

in a classical sense, i.e., we obtain with probability one a classical solution $u_{\varepsilon} \in$ $C_{x, t}^{2,1}\left(\bar{D} \times\left[0, T^{*}\right]\right) \cap C^{\infty}\left(D \times\left(0, T^{*}\right)\right)$. It is then immediate from the choice of $a_{\varepsilon}$ and the regularity of $u_{\varepsilon}$ that the equations (12)-(14) are satisfied pointwise everywhere. We can infer from the maximum principle that $\varepsilon \leq u_{\varepsilon}(x, t) \leq \varepsilon+\left\|u_{0}\right\|_{L^{\infty}(D)}$ holds true for all $(x, t) \in \bar{D} \times\left[0, T^{*}\right]$ as it is asserted in (15). The derivation of the energy estimate (16) is standard: multiply the equation with $u_{\varepsilon}$, integrate over $D$ and use the regularity of $u_{\varepsilon}$ to integrate by parts in the spatial differential operators. Note in this respect that as a consequence of (15) and (14) it holds $\mathbf{n}_{\partial D} \cdot \nabla u_{\varepsilon}^{m} \leq 0$ on $\partial D$, where 
$\mathbf{n}_{\partial D}$ is the exterior unit normal vector field along the $C^{2}$ manifold $\partial D$. This is the only reason for the inequality sign in (16) as we may compute for the second term

$$
\begin{aligned}
\int_{D} \frac{\mathrm{d}}{\mathrm{d} t} B_{t}^{\varepsilon} \cdot u_{\varepsilon} \nabla u_{\varepsilon} \mathrm{d} x & =\int_{D} \frac{\mathrm{d}}{\mathrm{d} t} B_{t}^{\varepsilon} \cdot u_{\varepsilon} \nabla\left(u_{\varepsilon}-\varepsilon\right) \mathrm{d} x \\
& =-\int_{D} \frac{\mathrm{d}}{\mathrm{d} t} B_{t}^{\varepsilon} \cdot\left(u_{\varepsilon}-\varepsilon\right) \nabla u_{\varepsilon}=-\int_{D} \frac{\mathrm{d}}{\mathrm{d} t} B_{t}^{\varepsilon} \cdot \frac{1}{2} \nabla\left|u_{\varepsilon}-\varepsilon\right|^{2}=0 .
\end{aligned}
$$

We proceed with the bound (17) for the time derivative. Multiplying the equation (12) with $\partial_{t} u_{\varepsilon}^{m}$, integrating over $D$, performing an integration by parts in the term with the porous medium operator and estimating the transport term by Hölder's and Young's inequality yields for all $t \in\left(0, T^{*}\right)$ the estimate

$$
\begin{aligned}
& \int_{D} m u_{\varepsilon}^{m-1}(t)\left|\partial_{t} u_{\varepsilon}\right|^{2}(t) \mathrm{d} x+\frac{\mathrm{d}}{\mathrm{d} t} \int_{D} \frac{1}{2}\left|\nabla u_{\varepsilon}^{m}\right|^{2}(t) \mathrm{d} x \\
& \quad \leq \frac{1}{2} \int_{D} m u_{\varepsilon}^{m-1}(t)\left|\partial_{t} u_{\varepsilon}\right|^{2}(t) \mathrm{d} x+\frac{v^{2}}{2} \int_{D}\left|\frac{\mathrm{d}}{\mathrm{d} t} B_{t}^{\varepsilon}\right|^{2} m u_{\varepsilon}^{m-1}(t)\left|\nabla u_{\varepsilon}\right|^{2}(t) \mathrm{d} x .
\end{aligned}
$$

Multiplying this bound with $t$ and integrating the resulting estimate over $(0, T)$ we may infer using also (16) and (15)

$$
\begin{aligned}
& \int_{0}^{T} \int_{D} \frac{t}{2} m u_{\varepsilon}^{m-1}\left|\partial_{t} u_{\varepsilon}\right|^{2} \mathrm{~d} x \mathrm{~d} t+\frac{T}{2} \int_{D}\left|\nabla u_{\varepsilon}^{m}\right|^{2}(T) \mathrm{d} x \\
& \quad \leq \frac{1}{2} \int_{0}^{T} \int_{D}\left|\nabla u_{\varepsilon}^{m}\right|^{2} \mathrm{~d} x \mathrm{~d} t+\sup _{0 \leq t \leq T^{*}}\left|\frac{\mathrm{d}}{\mathrm{d} t} B_{t}^{\varepsilon}\right|^{2} \frac{T^{*} v^{2}}{2} \int_{0}^{T} \int_{D} m u_{\varepsilon}^{m-1}\left|\nabla u_{\varepsilon}\right|^{2} \mathrm{~d} x \mathrm{~d} t \\
& \quad \leq\left(\frac{m}{2}\left(\varepsilon+\left\|u_{0}\right\|_{L^{\infty}}\right)^{m-1}+\frac{T^{*} v^{2}}{2} \sup _{0 \leq t \leq T^{*}}\left|\frac{\mathrm{d}}{\mathrm{d} t} B_{t}^{\varepsilon}\right|^{2}\right) \int_{D} \frac{1}{2}\left|u_{\varepsilon}\right|^{2}(0) \mathrm{d} x
\end{aligned}
$$

for all $T \in\left(0, T^{*}\right)$. Moreover, it follows from (7) and Doob's maximal inequality that $\mathbf{E} \sup _{0 \leq t \leq T^{*}}\left|\frac{\mathrm{d}}{\mathrm{d} t} B_{t}^{\varepsilon}\right|^{2} \leq C \varepsilon^{-\beta} T^{*} \mathbf{E}\left|B_{T^{*}}\right|^{2}$ for some absolute constant $C>0$. This establishes the estimate (17). The bound (18) is now a consequence of plugging in the equation (12), then using the triangle inequality, estimating the term with the time derivative by means of (17) and bounding the transport term similarly as at the end of the proof of (17). This concludes the proof of Lemma 4.

4.2 Proof of Proposition 5 (Contraction principle up to time-dependent shift for truncated Wong-Zakai type approximations)

Fix $\kappa>0$ and let $\zeta_{\delta}$ denote the smooth and convex approximation to the positive part truncation $r \mapsto r_{+}:=r \vee 0$ on scale $\delta>0$ such that (19)-(21) hold true. Define $\zeta_{\kappa}^{\delta}(r):=\kappa+\zeta_{\delta}(r-\kappa)$ which is a smooth and convex approximation to the truncation $r \mapsto r \vee \kappa$. Finally, fix $\varepsilon, \hat{\varepsilon} \leq \frac{\kappa}{2}$ and abbreviate for what follows $v_{\varepsilon}:=\zeta_{\kappa}^{\varepsilon^{q}} \circ u_{\varepsilon}^{\leftarrow}$ resp. $v_{\hat{\varepsilon}}:=\zeta_{\kappa}^{\hat{\varepsilon}^{q}} \circ u_{\hat{\varepsilon}}^{\leftarrow}$, where $q>1$ will be a large exponent to be specified later on 
in the proof. See (22) for the definition of the shifted densities. Finally, fix a compact set $K \subset D$.

We aim to derive an estimate for $\sup _{T \in\left[0, T^{*}\right]} \mathbf{E}\left\|v_{\mathcal{\varepsilon}}-v_{\hat{\varepsilon}}\right\|_{L^{1}(K)}$ of the same type as the asserted bound (23). The proof of this proceeds in several steps. For the sake of better readability, let us occasionally break the proof into intermediate results.

Lemma 8 Let the assumptions and notation of Sect. 4.2 be in place. We next choose a smooth, even and convex map $\eta: \mathbf{R} \rightarrow[0, \infty)$ such that $\eta(r)=|r|-1$ for $|r| \geq 2$ and $\eta(r) \leq|r|$ for all $r \in \mathbf{R}$. Define $\eta_{\delta}(r):=\delta \eta\left(\frac{r}{\delta}\right)$. There is a constant $C>0$ independent of $\delta$ such that

$$
\begin{aligned}
& \sup _{r \in \mathbf{R}}\left|\left(\eta_{\delta}\right)^{\prime}(r)\right|+|r|\left(\eta_{\delta}\right)^{\prime \prime}(r) \leq C, \\
& \left(\eta_{\delta}\right)^{\prime}(r) \rightarrow \operatorname{sign}(r) \text { and } \eta_{\delta}(r) \nearrow|r| \text { as } \delta \rightarrow 0, \\
& \left|\eta_{\delta}(r)-\right| r|| \leq C \delta \text { for all } r \in \mathbf{R} \text {, and } \eta_{\delta}(r)=|r|-\delta \text { for all }|r| \geq 2 \delta .
\end{aligned}
$$

Then the following "entropy estimate" holds true

$$
\begin{aligned}
& -\mathbf{E} \int_{0}^{T^{*}} \int_{\mathbf{R}^{d}} \eta_{\delta}\left(v_{\mathcal{E}}(x, t)-\hat{\mathcal{Z}}\right) \partial_{t} \xi(x, t) \mathrm{d} x \mathrm{~d} t \\
& \leq-\mathbf{E} \int_{0}^{T^{*}} \int_{\mathbf{R}^{d}}\left|\nabla v_{\varepsilon}^{m}(x, t)\right|^{2}\left(\eta_{\delta}\right)^{\prime \prime}\left(v_{\varepsilon}^{m}(x, t)-\hat{\mathcal{Z}}^{m}\right) \xi(x, t) \mathrm{d} x \mathrm{~d} t \\
& +\mathbf{E} \int_{0}^{T^{*}} \int_{\mathbf{R}^{d}} \eta_{\delta}\left(v_{\varepsilon}^{m}(x, t)-\hat{\mathcal{Z}}^{m}\right) \Delta \xi(x, t) \mathrm{d} x \mathrm{~d} t \\
& -\mathbf{E} \int_{0}^{T^{*}} \int_{\mathbf{R}^{d}} \frac{1}{2} v^{2}\left|\nabla v_{\varepsilon}(x, t)\right|^{2}\left(\eta_{\delta}\right)^{\prime \prime}\left(v_{\varepsilon}(x, t)-\hat{\mathcal{Z}}\right) \xi(x, t) \mathrm{d} x \mathrm{~d} t \\
& +\mathbf{E} \int_{0}^{T^{*}} \int_{\mathbf{R}^{d}} \frac{1}{2} v^{2} \eta_{\delta}\left(v_{\varepsilon}(x, t)-\hat{\mathcal{Z}}\right) \Delta \xi(x, t) \mathrm{d} x \mathrm{~d} t \\
& -\left.\mathbf{E} \int_{0}^{T^{*}} \int_{\mathbf{R}^{d}} v \eta_{\delta}\left(v_{\varepsilon}(x, t)-\hat{z}\right) \nabla \xi(x, t) \mathrm{d} x \mathrm{~d} B_{t}\right|_{\hat{z}=\hat{\mathcal{Z}}} \\
& +\mathbf{E} \int_{0}^{T^{*}} \int_{\mathbf{R}^{d}} \Delta v_{\varepsilon}^{m}(x, t) \xi(x, t)\left\{\left(\eta_{\delta}\right)^{\prime}\left(v_{\varepsilon}(x, t)-\hat{\mathcal{Z}}\right)-\left(\eta_{\delta}\right)^{\prime}\left(v_{\varepsilon}^{m}(x, t)-\hat{\mathcal{Z}}^{m}\right)\right\} \mathrm{d} x \mathrm{~d} t \\
& -\mathbf{E} \int_{0}^{T^{*}} \int_{\mathbf{R}^{d}} m\left(\left(u_{\varepsilon}^{\leftarrow}\right)^{m-1}-v_{\varepsilon}^{m-1}\right)(x, t) \nabla v_{\varepsilon}(x, t) \cdot\left(\eta_{\delta}\right)^{\prime}\left(v_{\varepsilon}(x, t)-\hat{\mathcal{Z}}\right) \nabla \xi(x, t) \mathrm{d} x \mathrm{~d} t
\end{aligned}
$$

for all $\xi \in C_{\mathrm{cpt}}^{\infty}\left(\mathbf{R}^{d} \times\left(0, T^{*}\right) ;[0, \infty)\right)$ and all bounded random variables $\hat{\mathcal{Z}} \in$ $L^{\infty}(\Omega)$. An analogous estimate holds true for $v_{\hat{\varepsilon}}$, see (49) below for the precise statement.

Proof Step 1 (Equation for $u_{\varepsilon}^{\leftarrow}$ and $u_{\hat{\varepsilon}}^{\leftarrow}$ ): The first step is to derive the equation for the shifted densities $u_{\varepsilon}^{\leftarrow}$ and $u_{\hat{\varepsilon}}^{\leftarrow}$, respectively. To this end, we aim to apply Itô's formula with respect to $\int_{\mathbf{R}^{d}} u_{\varepsilon}(x, t) \eta\left(x-v\left(B_{t}-B_{t}^{\varepsilon}\right), t\right) \mathrm{d} x$, where $\eta$ is an $\mathbf{F}$-adapted random test function $\eta \in C_{\mathrm{cpt}}^{\infty}\left(\mathbf{R}^{d} \times\left(0, T^{*}\right)\right)$. Note that $\partial_{t} u_{\varepsilon} \equiv 0$ on the lateral 
boundary $\partial D \times\left(0, T^{*}\right)$. Hence, it holds $\partial_{t} u_{\varepsilon} \in C\left(\mathbf{R}^{d} \times\left(0, T^{*}\right)\right)$ and we thus obtain from an application of Itô's formula for each $\mathbf{F}$-adapted random test function $\eta \in$ $C_{\mathrm{cpt}}^{\infty}\left(\mathbf{R}^{d} \times\left(0, T^{*}\right)\right)$ with probability one

$$
\begin{aligned}
& -\int_{0}^{T^{*}} \int_{\mathbf{R}^{d}} u_{\varepsilon}(x, t) \partial_{t} \eta\left(x-v\left(B_{t}-B_{t}^{\varepsilon}\right), t\right) \mathrm{d} x \mathrm{~d} t \\
& =\int_{0}^{T^{*}} \int_{\mathbf{R}^{d}} \partial_{t} u_{\varepsilon}(x, t) \eta\left(x-v\left(B_{t}-B_{t}^{\varepsilon}\right), t\right) \mathrm{d} x \mathrm{~d} t \\
& \quad+\int_{0}^{T^{*}} \int_{\mathbf{R}^{d}} u_{\varepsilon}(x, t) v \frac{\mathrm{d}}{\mathrm{d} t} B_{t}^{\varepsilon} \cdot \nabla \eta\left(x-v\left(B_{t}-B_{t}^{\varepsilon}\right), t\right) \mathrm{d} x \mathrm{~d} t \\
& \quad+\int_{0}^{T^{*}} \int_{\mathbf{R}^{d}} u_{\varepsilon}(x, t) \frac{1}{2} v^{2} \Delta \eta\left(x-v\left(B_{t}-B_{t}^{\varepsilon}\right), t\right) \mathrm{d} x \mathrm{~d} t \\
& \quad-\int_{0}^{T^{*}} \int_{\mathbf{R}^{d}} u_{\varepsilon}(x, t) v \nabla \eta\left(x-v\left(B_{t}-B_{t}^{\varepsilon}\right), t\right) \mathrm{d} x \mathrm{~d} B_{t} .
\end{aligned}
$$

Since $u_{\varepsilon}$ solves the equation (12) classically in $D \times\left(0, T^{*}\right)$, and is by definition constant outside of it, we may compute

$$
\begin{aligned}
& \int_{0}^{T^{*}} \int_{\mathbf{R}^{d}} \partial_{t} u_{\varepsilon}(x, t) \eta\left(x-v\left(B_{t}-B_{t}^{\varepsilon}\right), t\right) \mathrm{d} x \mathrm{~d} t \\
& \quad=\int_{0}^{T^{*}} \int_{\mathbf{R}^{d} \backslash \partial D} \Delta u_{\varepsilon}^{m}(x, t) \eta\left(x-v\left(B_{t}-B_{t}^{\varepsilon}\right), t\right) \mathrm{d} x \mathrm{~d} t \\
& \quad+\int_{0}^{T^{*}} \int_{\mathbf{R}^{d}} \eta\left(x-v\left(B_{t}-B_{t}^{\varepsilon}\right), t\right) v \frac{\mathrm{d}}{\mathrm{d} t} B_{t}^{\varepsilon} \cdot \nabla u_{\varepsilon}(x, t) \mathrm{d} x \mathrm{~d} t .
\end{aligned}
$$

(Despite $\partial D$ having Lebesgue measure zero, the domain of integration in the first right hand side term is $\mathbf{R}^{d} \backslash \partial D$ since $\nabla u_{\varepsilon}^{m}$ may jump across the domain boundary $\partial D$ for all $i \in\{1, \ldots, d\}$, and hence $\partial_{i} \nabla u_{\varepsilon}^{m}$ may not exist in the sense of weak derivatives in $\mathbf{R}^{d} \times\left(0, T^{*}\right)$ for all $i \in\{1, \ldots, d\}$.) Integrating by parts in the second term on the right hand side of the latter identity as well as performing a change of variables $x \mapsto x+v\left(B_{t}-B_{t}^{\varepsilon}\right)$ yields

$$
\begin{aligned}
& \int_{0}^{T^{*}} \int_{\mathbf{R}^{d}} \partial_{t} u_{\varepsilon}(x, t) \eta\left(x-v\left(B_{t}-B_{t}^{\varepsilon}\right), t\right) \mathrm{d} x \mathrm{~d} t \\
& \quad+\int_{0}^{T^{*}} \int_{\mathbf{R}^{d}} u_{\varepsilon}(x, t) v \frac{\mathrm{d}}{\mathrm{d} t} B_{t}^{\varepsilon} \cdot \nabla \eta\left(x-v\left(B_{t}-B_{t}^{\varepsilon}\right), t\right) \mathrm{d} x \mathrm{~d} t \\
& \quad=\int_{0}^{T^{*}} \int_{\mathbf{R}^{d} \backslash\left(-v\left(B_{t}-B_{t}^{\varepsilon}\right)+\partial D\right)} \Delta\left(u_{\varepsilon}^{\leftarrow}\right)^{m}(x, t) \eta(x, t) \mathrm{d} x \mathrm{~d} t .
\end{aligned}
$$

Note that there is no boundary integral appearing from the integration by parts in the second term on the right hand side of (35) since $u_{\varepsilon} \in C\left(\mathbf{R}^{d} \times\left(0, T^{*}\right)\right)$. We compute 
analogously

$$
\begin{gathered}
\int_{0}^{T^{*}} \int_{\mathbf{R}^{d}} u_{\varepsilon}(x, t) \frac{1}{2} v^{2} \Delta \eta\left(x-v\left(B_{t}-B_{t}^{\varepsilon}\right), t\right) \mathrm{d} x \mathrm{~d} t \\
=-\int_{0}^{T^{*}} \int_{\mathbf{R}^{d}} \frac{1}{2} v^{2} \nabla u_{\varepsilon}^{\leftarrow}(x, t) \cdot \nabla \eta(x, t) \mathrm{d} x \mathrm{~d} t
\end{gathered}
$$

as well as

$$
\begin{gathered}
-\int_{0}^{T^{*}} \int_{\mathbf{R}^{d}} u_{\varepsilon}(x, t) v \nabla \eta\left(x-v\left(B_{t}-B_{t}^{\varepsilon}\right), t\right) \mathrm{d} x \mathrm{~d} B_{t} . \\
=\int_{0}^{T^{*}} \int_{\mathbf{R}^{d}} v \nabla u_{\varepsilon}^{\leftarrow}(x, t) \eta(x, t) \mathrm{d} x \mathrm{~d} B_{t} .
\end{gathered}
$$

From (34), (36), (37) and (38) we infer that for each $\mathbf{F}$-adapted random test function $\eta \in C_{\mathrm{cpt}}^{\infty}\left(\mathbf{R}^{d} \times\left(0, T^{*}\right)\right)$ it holds with probability one

$$
\begin{aligned}
- & \int_{0}^{T^{*}} \int_{\mathbf{R}^{d}} u_{\varepsilon}^{\leftarrow}(x, t) \partial_{t} \eta(x, t) \mathrm{d} x \mathrm{~d} t \\
= & \int_{0}^{T^{*}} \int_{\mathbf{R}^{d} \backslash\left(-v\left(B_{t}-B_{t}^{\varepsilon}\right)+\partial D\right)} \Delta\left(u_{\varepsilon}^{\leftarrow}\right)^{m}(x, t) \eta(x, t) \mathrm{d} x \mathrm{~d} t \\
& -\int_{0}^{T^{*}} \int_{\mathbf{R}^{d}} \frac{1}{2} v^{2} \nabla u_{\varepsilon}^{\leftarrow}(x, t) \cdot \nabla \eta(x, t) \mathrm{d} x \mathrm{~d} t \\
& +\int_{0}^{T^{*}} \int_{\mathbf{R}^{d}} v \nabla u_{\varepsilon}^{\leftarrow}(x, t) \eta(x, t) \mathrm{d} x \mathrm{~d} B_{t} .
\end{aligned}
$$

Analogously one derives the equation for $u_{\hat{\varepsilon}}^{\leftarrow}$.

Step 2 (Convex approximation to $r \mapsto r \vee \kappa$ as test function): In the next step we aim to derive the equation for $v_{\varepsilon}=\zeta_{\kappa}^{\varepsilon^{q}} \circ u_{\varepsilon}^{\leftarrow}$ based on the equation for the shifted density derived in (39). The idea is to test the equation (39) with the test function $\eta:=$ $\left(\left(\zeta_{\kappa}^{\varepsilon^{q}}\right)^{\prime} \circ u_{\varepsilon}^{\leftarrow}\right) \xi$, where $\xi \in C_{\mathrm{cpt}}^{\infty}\left(\mathbf{R}^{d} \times\left(0, T^{*}\right) ;[0, \infty)\right)$ is arbitrary. However, since the shifted density $u_{\varepsilon}^{\leftarrow}$ is only Hölder continuous in the time variable we have to regularize first. To this end, we make use of the Steklov average $\eta_{h}(x, t):=\frac{1}{h} \int_{t-h}^{t} \eta(x, s) \mathrm{d} s$ which is an admissible test function for (39) for all sufficiently small $h>0$ (depending only on the support of $\xi$ ). Since $\partial_{t} \eta_{h}(x, t)=\frac{\eta(x, t)-\eta(x, t-h)}{h}$ we obtain by a simple change of variables

$$
\begin{aligned}
& -\int_{0}^{T^{*}} \int_{\mathbf{R}^{d}} u_{\varepsilon}^{\leftarrow}(x, t) \partial_{t} \eta_{h}(x, t) \mathrm{d} x \mathrm{~d} t \\
& =-\int_{0}^{T^{*}} \int_{\mathbf{R}^{d}} \frac{1}{h}\left(u_{\varepsilon}^{\leftarrow}(x, t+h)-u_{\varepsilon}^{\leftarrow}(x, t)\right)\left(\zeta_{\kappa}^{\varepsilon^{q}}\right)^{\prime}\left(u_{\varepsilon}^{\leftarrow}(x, t)\right) \xi(x, t) \mathrm{d} x \mathrm{~d} t
\end{aligned}
$$


for each $h>0$ and each $\xi \in C_{\mathrm{cpt}}^{\infty}\left(\mathbf{R}^{d} \times\left(0, T^{*}\right) ;[0, \infty)\right)$ almost surely. We then deduce from the smoothness and convexity of $\zeta_{\kappa}^{\varepsilon^{q}}$ as well as by reverting the change of variables the bound

$$
\begin{aligned}
& -\int_{0}^{T^{*}} \int_{\mathbf{R}^{d}} u_{\varepsilon}^{\leftarrow}(x, t) \partial_{t} \eta_{h}(x, t) \mathrm{d} x \mathrm{~d} t \\
& \geq-\int_{0}^{T^{*}} \int_{\mathbf{R}^{d}} \frac{1}{h}\left(\zeta_{\kappa}^{\varepsilon^{q}}\left(u_{\varepsilon}^{\leftarrow}(x, t+h)\right)-\zeta_{\kappa}^{\varepsilon^{q}}\left(u_{\varepsilon}^{\leftarrow}(x, t)\right)\right) \xi(x, t) \mathrm{d} x \mathrm{~d} t \\
& =-\int_{0}^{T^{*}} \int_{\mathbf{R}^{d}} \frac{1}{h}(\xi(x, t-h)-\xi(x, t)) \zeta_{\kappa}^{\varepsilon^{q}}\left(u_{\varepsilon}^{\leftarrow}(x, t)\right) \mathrm{d} x \mathrm{~d} t
\end{aligned}
$$

for each $h>0$ and each $\xi \in C_{\mathrm{cpt}}^{\infty}\left(D \times\left(0, T^{*}\right) ;[0, \infty)\right)$ almost surely. Hence, we may infer from this, (39) and standard properties of the Steklov average after letting $h \rightarrow 0$ the estimate

$$
\begin{aligned}
- & \int_{0}^{T^{*}} \int_{\mathbf{R}^{d}} \zeta_{\mathcal{\varepsilon}}^{\varepsilon^{q}}\left(u_{\varepsilon}^{\leftarrow}(x, t)\right) \partial_{t} \xi(x, t) \mathrm{d} x \mathrm{~d} t \\
\leq & \int_{0}^{T^{*}} \int_{\mathbf{R}^{d} \backslash\left(-v\left(B_{t}-B_{t}^{\varepsilon}\right)+\partial D\right)} \Delta\left(u_{\varepsilon}^{\leftarrow}\right)^{m}(x, t)\left(\zeta_{\kappa}^{\varepsilon^{q}}\right)^{\prime}\left(u_{\varepsilon}^{\leftarrow}(x, t)\right) \xi(x, t) \mathrm{d} x \mathrm{~d} t \\
& -\int_{0}^{T^{*}} \int_{\mathbf{R}^{d}} \frac{1}{2} v^{2} \nabla u_{\varepsilon}^{\leftarrow}(x, t) \cdot \nabla\left(\left(\zeta_{\kappa}^{\varepsilon^{q}}\right)^{\prime}\left(u_{\varepsilon}^{\leftarrow}(x, t)\right) \xi(x, t)\right) \mathrm{d} x \mathrm{~d} t \\
& +\int_{0}^{T^{*}} \int_{\mathbf{R}^{d}} v \nabla u_{\varepsilon}^{\leftarrow}(x, t)\left(\zeta_{\kappa}^{\varepsilon^{q}}\right)^{\prime}\left(u_{\varepsilon}^{\leftarrow}(x, t)\right) \xi(x, t) \mathrm{d} x \mathrm{~d} B_{t},
\end{aligned}
$$

which is valid for each $\xi \in C_{\mathrm{cpt}}^{\infty}\left(\mathbf{R}^{d} \times\left(0, T^{*}\right) ;[0, \infty)\right)$ on a set with probability one. Note that on $\mathbf{R}^{d} \backslash\left(-v\left(B_{t}-B_{t}^{\varepsilon}\right)+\partial D\right)$ we may apply the chain rule to compute that $\nabla u_{\varepsilon}^{\leftarrow}(x, t)\left(\zeta_{\kappa}^{\varepsilon^{q}}\right)^{\prime}\left(u_{\varepsilon}^{\leftarrow}(x, t)\right)=\nabla\left(\zeta_{\kappa}^{\varepsilon^{q}} \circ u_{\varepsilon}^{\leftarrow}\right)(x, t)$. Furthermore, note that $\left(\zeta_{\kappa}^{\varepsilon^{q}}\right)^{\prime}(r)=$ 0 holds true for all $r \leq \kappa$. In particular, because of $u_{\varepsilon}^{\leftarrow} \in C\left(\mathbf{R}^{d} \times\left[0, T^{*}\right]\right)$ and the choice $\varepsilon \leq \frac{\kappa}{2}$ we have for all $t \in\left[0, T^{*}\right]$ that $\left(\zeta_{\kappa}^{\varepsilon^{q}}\right)^{\prime} \circ u_{\varepsilon}^{\leftarrow}(\cdot, t) \equiv 0$ in a neighborhood of the interface $\left(-v\left(B_{t}-B_{t}^{\varepsilon}\right)+\partial D\right)$. This in turn means that we can integrate by parts in the first term on the right hand side of (41) without producing an additional surface integral. Taking all of these information together yields the bound

$$
\begin{aligned}
- & \int_{0}^{T^{*}} \int_{\mathbf{R}^{d}}\left(\zeta_{\kappa}^{\varepsilon^{q}} \circ u_{\varepsilon}^{\leftarrow}\right) \partial_{t} \xi \mathrm{d} x \mathrm{~d} t \\
\leq & -\int_{0}^{T^{*}} \int_{\mathbf{R}^{d}} m\left(u_{\varepsilon}^{\leftarrow}\right)^{m-1}\left|\nabla u_{\varepsilon}^{\leftarrow}\right|^{2}\left(\left(\zeta_{\kappa}^{\varepsilon^{q}}\right)^{\prime \prime} \circ u_{\varepsilon}^{\leftarrow}\right) \xi \mathrm{d} x \mathrm{~d} t \\
& -\int_{0}^{T^{*}} \int_{\mathbf{R}^{d}} m\left(u_{\varepsilon}^{\leftarrow}\right)^{m-1} \nabla\left(\zeta_{\kappa}^{\varepsilon^{q}} \circ u_{\varepsilon}^{\leftarrow}\right) \cdot \nabla \xi \mathrm{d} x \mathrm{~d} t \\
& -\int_{0}^{T^{*}} \int_{\mathbf{R}^{d}} \frac{1}{2} v^{2}\left|\nabla u_{\varepsilon}^{\leftarrow}\right|^{2}\left(\left(\zeta_{\kappa}^{\varepsilon^{q}}\right)^{\prime \prime} \circ u_{\varepsilon}^{\leftarrow}\right) \xi \mathrm{d} x \mathrm{~d} t
\end{aligned}
$$




$$
\begin{aligned}
& -\int_{0}^{T^{*}} \int_{\mathbf{R}^{d}} \frac{1}{2} v^{2} \nabla\left(\zeta_{\kappa}^{\varepsilon^{q}} \circ u_{\varepsilon}^{\leftarrow}\right) \cdot \nabla \xi \mathrm{d} x \mathrm{~d} t \\
& +\int_{0}^{T^{*}} \int_{\mathbf{R}^{d}} v \nabla\left(\zeta_{\kappa}^{\varepsilon^{q}} \circ u_{\varepsilon}^{\leftarrow}\right) \xi \mathrm{d} x \mathrm{~d} B_{t}
\end{aligned}
$$

for each $\xi \in C_{\mathrm{cpt}}^{\infty}\left(\mathbf{R}^{d} \times\left(0, T^{*}\right) ;[0, \infty)\right)$ almost surely. Exploiting the sign $\left(\zeta_{\kappa}^{\varepsilon^{q}}\right)^{\prime \prime} \geq 0$ and making use of the abbreviation $v_{\varepsilon}=\zeta_{\kappa}^{\varepsilon^{q}} \circ u_{\varepsilon}^{\leftarrow}$ we arrive at the estimate

$$
\begin{aligned}
- & \int_{0}^{T^{*}} \int_{\mathbf{R}^{d}} v_{\varepsilon}(x, t) \partial_{t} \xi(x, t) \mathrm{d} x \mathrm{~d} t \\
\leq & -\int_{0}^{T^{*}} \int_{\mathbf{R}^{d}} \nabla v_{\varepsilon}^{m}(x, t) \cdot \nabla \xi(x, t) \mathrm{d} x \mathrm{~d} t \\
& -\int_{0}^{T^{*}} \int_{\mathbf{R}^{d}} \frac{1}{2} v^{2} \nabla v_{\varepsilon}(x, t) \cdot \nabla \xi(x, t) \mathrm{d} x \mathrm{~d} t \\
& +\int_{0}^{T^{*}} \int_{\mathbf{R}^{d}} v \nabla v_{\varepsilon}(x, t) \xi(x, t) \mathrm{d} x \mathrm{~d} B_{t} \\
& -\int_{0}^{T^{*}} \int_{\mathbf{R}^{d}} m\left(\left(u_{\varepsilon}^{\leftarrow}\right)^{m-1}-v_{\varepsilon}^{m-1}\right)(x, t) \nabla v_{\varepsilon}(x, t) \cdot \nabla \xi(x, t) \mathrm{d} x \mathrm{~d} t
\end{aligned}
$$

which is valid for all $\xi \in C_{\mathrm{cpt}}^{\infty}\left(\mathbf{R}^{d} \times\left(0, T^{*}\right) ;[0, \infty)\right)$ almost surely. An analogous estimate also holds true for the pair $\left(u_{\hat{\varepsilon}}^{\leftarrow}, v_{\hat{\varepsilon}}=\zeta_{\kappa}^{\hat{\varepsilon}^{q}} \circ u_{\hat{\varepsilon}}^{\leftarrow}\right)$, i.e.,

$$
\begin{aligned}
- & \int_{0}^{T^{*}} \int_{\mathbf{R}^{d}} v_{\hat{\varepsilon}}(y, s) \partial_{t} \tilde{\xi}(y, s) \mathrm{d} y \mathrm{~d} s \\
\leq & -\int_{0}^{T^{*}} \int_{\mathbf{R}^{d}} \nabla v_{\hat{\varepsilon}}^{m}(y, s) \cdot \nabla \tilde{\xi}(y, s) \mathrm{d} y \mathrm{~d} s \\
& -\int_{0}^{T^{*}} \int_{\mathbf{R}^{d}} \frac{1}{2} v^{2} \nabla v_{\hat{\varepsilon}}(y, s) \cdot \nabla \tilde{\xi}(y, s) \mathrm{d} y \mathrm{~d} s \\
& +\int_{0}^{T^{*}} \int_{\mathbf{R}^{d}} v \nabla v_{\hat{\varepsilon}}(y, s) \tilde{\xi}(y, s) \mathrm{d} y \mathrm{~d} B_{s} \\
& -\int_{0}^{T^{*}} \int_{\mathbf{R}^{d}} m\left(\left(u_{\hat{\varepsilon}}^{\leftarrow}\right)^{m-1}-v_{\hat{\varepsilon}}^{m-1}\right)(y, s) \nabla v_{\hat{\varepsilon}}(y, s) \cdot \nabla \tilde{\xi}(y, s) \mathrm{d} y \mathrm{~d} s
\end{aligned}
$$

for all $\tilde{\xi} \in C_{\mathrm{cpt}}^{\infty}\left(D \times\left(0, T^{*}\right) ;[0, \infty)\right)$ almost surely.

Step 3 (Convex approximation $\eta_{\delta}$ to $r \mapsto|r|$ as test function): We proceed by testing the inequality (43) with $\left(\eta_{\delta}\right)^{\prime}\left(v_{\varepsilon}(x, t)-\hat{z}\right) \xi(x, t)$ where the test function $\xi \in$ $C_{\mathrm{cpt}}^{\infty}\left(\mathbf{R}^{d} \times\left(0, T^{*}\right) ;[0, \infty)\right)$ and $\hat{z} \in \mathbf{R}$ are arbitrary. This again incorporates several integration by parts which we do not want to produce any additional surface integrals. We reiterate that this will indeed not be the case since neither the Dirichlet data nor the Neumann data for $v_{\varepsilon}$ jump across the interfaces $-v\left(B_{t}-B_{t}^{\varepsilon}\right)+\partial D$ for all $t \in\left[0, T^{*}\right]$. 
Hence, arguing similar to the previous step using in particular the Steklov average and the convexity of $\eta_{\delta}$ we obtain the estimate

$$
\begin{aligned}
- & \int_{0}^{T^{*}} \int_{\mathbf{R}^{d}} \eta_{\delta}\left(v_{\varepsilon}(x, t)-\hat{z}\right) \partial_{t} \xi(x, t) \mathrm{d} x \mathrm{~d} t \\
\leq & -\int_{0}^{T^{*}} \int_{\mathbf{R}^{d}} \nabla v_{\varepsilon}^{m}(x, t) \cdot \nabla\left(\left(\eta_{\delta}\right)^{\prime}\left(v_{\varepsilon}(x, t)-\hat{z}\right) \xi(x, t)\right) \mathrm{d} x \mathrm{~d} t \\
& -\int_{0}^{T^{*}} \int_{\mathbf{R}^{d}} \frac{1}{2} v^{2} \nabla v_{\varepsilon}(x, t) \cdot \nabla\left(\left(\eta_{\delta}\right)^{\prime}\left(v_{\varepsilon}(x, t)-\hat{z}\right) \xi(x, t)\right) \mathrm{d} x \mathrm{~d} t \\
& -\int_{0}^{T^{*}} \int_{\mathbf{R}^{d}} v \eta_{\delta}\left(v_{\varepsilon}(x, t)-\hat{z}\right) \nabla \xi(x, t) \mathrm{d} x \mathrm{~d} B_{t} \\
& -\int_{0}^{T^{*}} \int_{\mathbf{R}^{d}} m\left(\left(u_{\varepsilon}^{\leftarrow}\right)^{m-1}-v_{\varepsilon}^{m-1}\right)(x, t)\left|\nabla v_{\varepsilon}(x, t)\right|^{2}\left(\eta_{\delta}\right)^{\prime \prime}\left(v_{\varepsilon}(x, t)-\hat{z}\right) \xi(x, t) \mathrm{d} x \mathrm{~d} t \\
& -\int_{0}^{T^{*}} \int_{\mathbf{R}^{d}} m\left(\left(u_{\varepsilon}^{\leftarrow}\right)^{m-1}-v_{\varepsilon}^{m-1}\right)(x, t) \nabla v_{\varepsilon}(x, t) \cdot\left(\eta_{\delta}\right)^{\prime}\left(v_{\varepsilon}(x, t)-\hat{z}\right) \nabla \xi(x, t) \mathrm{d} x \mathrm{~d} t
\end{aligned}
$$

for all $\xi \in C_{\mathrm{cpt}}^{\infty}\left(\mathbf{R}^{d} \times\left(0, T^{*}\right) ;[0, \infty)\right)$ and all $\hat{z} \in \mathbf{R}$. As a preparation for what follows, we post-process the right hand side of the latter inequality. Integrating by parts, adding zero and using the chain rule we may rewrite the non-linear diffusion term as follows

$$
\begin{aligned}
- & \int_{0}^{T^{*}} \int_{\mathbf{R}^{d}} \nabla v_{\varepsilon}^{m}(x, t) \cdot \nabla\left(\left(\eta_{\delta}\right)^{\prime}\left(v_{\varepsilon}(x, t)-\hat{z}\right) \xi(x, t)\right) \mathrm{d} x \mathrm{~d} t \\
= & -\int_{0}^{T^{*}} \int_{\mathbf{R}^{d}}\left|\nabla v_{\varepsilon}^{m}(x, t)\right|^{2}\left(\eta_{\delta}\right)^{\prime \prime}\left(v_{\varepsilon}^{m}(x, t)-\hat{z}^{m}\right) \xi(x, t) \mathrm{d} x \mathrm{~d} t \\
& +\int_{0}^{T^{*}} \int_{\mathbf{R}^{d}} \eta_{\delta}\left(v_{\varepsilon}^{m}(x, t)-\hat{z}^{m}\right) \Delta \xi(x, t) \mathrm{d} x \mathrm{~d} t \\
& +\int_{0}^{T^{*}} \int_{\mathbf{R}^{d}} \Delta v_{\varepsilon}^{m}(x, t) \xi(x, t)\left\{\left(\eta_{\delta}\right)^{\prime}\left(v_{\varepsilon}(x, t)-\hat{z}\right)-\left(\eta_{\delta}\right)^{\prime}\left(v_{\varepsilon}^{m}(x, t)-\hat{z}^{m}\right)\right\} \mathrm{d} x \mathrm{~d} t .
\end{aligned}
$$

Analogously one obtains for the Stratonovich correction term

$$
\begin{aligned}
& -\int_{0}^{T^{*}} \int_{\mathbf{R}^{d}} \frac{1}{2} v^{2} \nabla v_{\varepsilon}(x, t) \cdot \nabla\left(\left(\eta_{\delta}\right)^{\prime}\left(v_{\varepsilon}(x, t)-\hat{z}\right) \xi(x, t)\right) \mathrm{d} x \mathrm{~d} t \\
& =-\int_{0}^{T^{*}} \int_{\mathbf{R}^{d}} \frac{1}{2} v^{2}\left|\nabla v_{\varepsilon}(x, t)\right|^{2}\left(\eta_{\delta}\right)^{\prime \prime}\left(v_{\varepsilon}(x, t)-\hat{z}\right) \xi(x, t) \mathrm{d} x \mathrm{~d} t \\
& \quad+\int_{0}^{T^{*}} \int_{\mathbf{R}^{d}} \frac{1}{2} v^{2} \eta_{\delta}\left(v_{\varepsilon}(x, t)-\hat{z}\right) \Delta \xi(x, t) \mathrm{d} x \mathrm{~d} t .
\end{aligned}
$$


Since $\chi_{\operatorname{supp}} \nabla v_{\varepsilon} v_{\varepsilon} \leq \chi_{\operatorname{supp} \nabla v_{\varepsilon}} u_{\varepsilon}^{\leftarrow}$ by the definition of $v_{\varepsilon}=\zeta_{\kappa}^{\varepsilon^{q}} \circ u_{\varepsilon}^{\leftarrow}$ and the truncation $\zeta_{\kappa}^{\varepsilon^{q}}$ we observe that the penultimate term in (45) has a favorable sign. Together with the two identities (46) and (47) the bound (45) thus yields for all $\hat{z} \in \mathbf{R}$ and all $\xi \in C_{\mathrm{cpt}}^{\infty}\left(\mathbf{R}^{d} \times\left(0, T^{*}\right) ;[0, \infty)\right)$ almost surely the estimate

$$
\begin{aligned}
- & \int_{0}^{T^{*}} \int_{\mathbf{R}^{d}} \eta_{\delta}\left(v_{\varepsilon}(x, t)-\hat{z}\right) \partial_{t} \xi(x, t) \mathrm{d} x \mathrm{~d} t \\
\leq & -\int_{0}^{T^{*}} \int_{\mathbf{R}^{d}}\left|\nabla v_{\varepsilon}^{m}(x, t)\right|^{2}\left(\eta_{\delta}\right)^{\prime \prime}\left(v_{\varepsilon}^{m}(x, t)-\hat{z}^{m}\right) \xi(x, t) \mathrm{d} x \mathrm{~d} t \\
& +\int_{0}^{T^{*}} \int_{\mathbf{R}^{d}} \eta_{\delta}\left(v_{\varepsilon}^{m}(x, t)-\hat{z}^{m}\right) \Delta \xi(x, t) \mathrm{d} x \mathrm{~d} t \\
& -\int_{0}^{T^{*}} \int_{\mathbf{R}^{d}} \frac{1}{2} v^{2}\left|\nabla v_{\varepsilon}(x, t)\right|^{2}\left(\eta_{\delta}\right)^{\prime \prime}\left(v_{\varepsilon}(x, t)-\hat{z}\right) \xi(x, t) \mathrm{d} x \mathrm{~d} t \\
& +\int_{0}^{T^{*}} \int_{\mathbf{R}^{d}} \frac{1}{2} v^{2} \eta_{\delta}\left(v_{\varepsilon}(x, t)-\hat{z}\right) \Delta \xi(x, t) \mathrm{d} x \mathrm{~d} t \\
& -\int_{0}^{T^{*}} \int_{\mathbf{R}^{d}} v \eta_{\delta}\left(v_{\varepsilon}(x, t)-\hat{z}\right) \nabla \xi(x, t) \mathrm{d} x \mathrm{~d} B_{t} \\
& +\int_{0}^{T^{*}} \int_{\mathbf{R}^{d}} \Delta v_{\varepsilon}^{m}(x, t) \xi(x, t)\left\{\left(\eta_{\delta}\right)^{\prime}\left(v_{\varepsilon}(x, t)-\hat{z}\right)-\left(\eta_{\delta}\right)^{\prime}\left(v_{\varepsilon}^{m}(x, t)-\hat{z}^{m}\right)\right\} \mathrm{d} x \mathrm{~d} t \\
& -\int_{0}^{T^{*}} \int_{\mathbf{R}^{d}} m\left(\left(u_{\varepsilon}^{\leftarrow}\right)^{m-1}-v_{\varepsilon}^{m-1}\right)(x, t) \nabla v_{\varepsilon}(x, t) \cdot\left(\eta_{\delta}\right)^{\prime}\left(v_{\varepsilon}(x, t)-\hat{z}\right) \nabla \xi(x, t) \mathrm{d} x \mathrm{~d} t
\end{aligned}
$$

Step 4 (Substituting $\hat{z} \rightsquigarrow \hat{\mathcal{Z}}$ and taking expectation): We define for every $\lambda>0$ a smooth and compactly supported cut-off $\bar{\rho}_{\lambda}:=\frac{1}{\lambda} \bar{\rho}(\dot{\bar{\lambda}})$ by rescaling a standard even cut-off function $\bar{\rho} \in C_{\mathrm{cpt}}^{\infty}((-1,1) ;[0, \infty))$ such that $\int_{\mathbf{R}} \bar{\rho}(r) \mathrm{d} r=1$. We may then multiply the inequality (48) with the non-negative random variable $\rho_{\lambda}(\hat{z}-\hat{\mathcal{Z}})$, take the expected value of the resulting almost sure estimate, and finally integrate over $\hat{z} \in \mathbf{R}$. We claim that taking the limit $\lambda \rightarrow 0$ produces the desired estimate (33).

To this end, we focus on the parameter dependent stochastic integral term $\mathbf{R} \ni$ $\hat{z} \mapsto X(\hat{z}):=\int_{0}^{T^{*}} \int_{\mathbf{R}^{d}} v \eta_{\delta}\left(v_{\varepsilon}(x, t)-\hat{z}\right) \nabla \xi(x, t) \mathrm{d} x \mathrm{~d} B_{t}$. Note that because of the maximum principle bound (15), the definition $v_{\varepsilon}=\zeta_{\kappa}^{\varepsilon^{q}} \circ u_{\varepsilon}^{\leftarrow}$, as well as the Burkholder-Davis-Gundy inequality it holds $\mathbf{E}\left|X^{\prime}(0)\right|+\mathbf{E}\left|X^{\prime \prime}(0)\right|<\infty$. Hence, $\left.\mathbf{E}|X(\hat{z})|_{\hat{z}=\hat{\mathcal{Z}}}\left|\leq\|\hat{\mathcal{Z}}\|_{L^{\infty}} \mathbf{E}\right| X^{\prime}(0)|<\infty, \mathbf{E}| X^{\prime}(\hat{z})\right|_{\hat{z}=\hat{\mathcal{Z}}}\left|\leq\|\hat{\mathcal{Z}}\|_{L^{\infty}} \mathbf{E}\right| X^{\prime \prime}(0) \mid<\infty$, and

$$
\mathbf{E}|X(\hat{z})|_{\hat{z}=\hat{\mathcal{Z}}}-\left.\int_{\mathbf{R}} \rho_{\lambda}(\hat{z}-\hat{\mathcal{Z}}) X(\hat{z}) \mathrm{d} \hat{z}\left|\leq \int_{\mathbf{R}} \hat{z} \rho_{\lambda}(\hat{z}) \mathrm{d} \hat{z} \mathbf{E}\right| X^{\prime}(\hat{z})\right|_{\hat{z}=\hat{\mathcal{Z}}} \mid \rightarrow 0 \text { as } \lambda \rightarrow 0 \text {. }
$$

This yields the claim for the noise term appearing in (48). We observe that all the other terms can be dealt with based on the estimates (15)-(18) from Lemma 4, which in turn concludes the proof of (33). 
Finally, by taking (44) instead of (43) as a starting point for the previous two steps we obtain along the same lines that

$$
\begin{aligned}
- & \mathbf{E} \int_{0}^{T^{*}} \int_{\mathbf{R}^{d}} \eta_{\delta}\left(v_{\hat{\varepsilon}}(y, s)-\mathcal{Z}\right) \partial_{t} \tilde{\xi}(y, s) \mathrm{d} y \mathrm{~d} s \\
\leq & -\mathbf{E} \int_{0}^{T^{*}} \int_{\mathbf{R}^{d}}\left|\nabla v_{\hat{\varepsilon}}^{m}(y, s)\right|^{2}\left(\eta_{\delta}\right)^{\prime \prime}\left(v_{\hat{\varepsilon}}^{m}(y, s)-\mathcal{Z}^{m}\right) \tilde{\xi}(y, s) \mathrm{d} y \mathrm{~d} s \\
& +\mathbf{E} \int_{0}^{T^{*}} \int_{\mathbf{R}^{d}} \eta_{\delta}\left(v_{\hat{\varepsilon}}^{m}(y, s)-\mathcal{Z}^{m}\right) \Delta \tilde{\xi}(y, s) \mathrm{d} y \mathrm{~d} s \\
& -\mathbf{E} \int_{0}^{T^{*}} \int_{\mathbf{R}^{d}} \frac{1}{2} v^{2}\left|\nabla v_{\hat{\varepsilon}}(y, s)\right|^{2}\left(\eta_{\delta}\right)^{\prime \prime}\left(v_{\hat{\varepsilon}}(y, s)-\mathcal{Z}\right) \tilde{\xi}(y, s) \mathrm{d} y \mathrm{~d} s \\
& +\mathbf{E} \int_{0}^{T^{*}} \int_{\mathbf{R}^{d}} \frac{1}{2} v^{2} \eta_{\delta}\left(v_{\hat{\varepsilon}}(y, s)-\mathcal{Z}\right) \Delta \tilde{\xi}(y, s) \mathrm{d} y \mathrm{~d} s \\
& -\left.\mathbf{E} \int_{0}^{T^{*}} \int_{\mathbf{R}^{d}} v \eta_{\delta}\left(v_{\hat{\varepsilon}}(y, s)-z\right) \nabla \tilde{\xi}(y, s) \mathrm{d} y \mathrm{~d} B_{s}\right|_{z=\mathcal{Z}} \\
& +\mathbf{E} \int_{0}^{T^{*}} \int_{\mathbf{R}^{d}} \Delta v_{\hat{\varepsilon}}^{m}(y, s) \tilde{\xi}(y, s)\left\{(\eta \delta)^{\prime}\left(v_{\hat{\varepsilon}}(y, s)-\mathcal{Z}\right)-\left(\eta_{\delta}\right)^{\prime}\left(v_{\hat{\varepsilon}}^{m}(y, s)-\mathcal{Z}^{m}\right)\right\} \mathrm{d} y \mathrm{~d} s \\
& -\mathbf{E} \int_{0}^{T^{*}} \int_{\mathbf{R}^{d}} m\left(\left(u_{\hat{\varepsilon}}\right)^{m-1}-v_{\hat{\varepsilon}}^{m-1}\right)(y, s) \nabla v_{\hat{\varepsilon}}(y, s) \cdot\left(\eta_{\delta}\right)^{\prime}\left(v_{\hat{\varepsilon}}(y, s)-\mathcal{Z}\right) \nabla \tilde{\xi}(y, s) \mathrm{d} y \mathrm{~d} s,
\end{aligned}
$$

for all test functions $\tilde{\xi} \in C_{\mathrm{cpt}}^{\infty}\left(\mathbf{R}^{d} \times\left(0, T^{*}\right) ;[0, \infty)\right)$, as well as all bounded random variables $\mathcal{Z}$. This concludes the proof of Lemma 8 .

We continue with the proof of Proposition 5. The next step takes care of the proper choice of test functions $\xi(x, t)$ resp. $\tilde{\xi}(y, s)$ in the latter two estimates. After that, we start merging them by $i$ ) substituting $\hat{\mathcal{Z}}=v_{\hat{\varepsilon}}(y, s)$ in (33) resp. $\mathcal{Z}=v_{\varepsilon}(x, t)$ in (49), ii) integrating over the respective independent variables $(y, s)$ resp. $(x, t)$, and iii) summing the two resulting inequalities.

Consider the mollifier $\rho \in C_{\mathrm{cpt}}^{\infty}((0,1) ;[0, \infty))$ with $\int_{\mathbf{R}} \rho(r) \mathrm{d} r$ already used in (7), and define for $\tau>0$ the scaled kernel $\rho_{\tau}:=\frac{1}{\tau} \rho\left(\frac{\dot{\tau}}{\tau}\right)$. Let $\varphi \in C_{\mathrm{cpt}}^{\infty}\left(\left(0, T^{*}\right) ;[0,1]\right)$ and fix another even mollifier $\gamma \in C_{\mathrm{cpt}}^{\infty}\left(B_{1} ;[0, \infty)\right)$ such that $\int_{B_{1}} \gamma(x) \mathrm{d} x=1$. For $\theta>0$ let $\gamma_{\theta}:=\frac{1}{\theta^{d}} \gamma(\dot{\dot{\theta}})$. Now, since $K \subset D$ is compact we can find a scale $s_{c} \in(0,1)$ such that $K$ is contained in $D_{s_{c}}:=\left\{x \in D: \operatorname{dist}(x, \partial D)>s_{c}\right\}$. Moreover, because $D$ has a $C^{2}$ boundary $\partial D$ there exists (cf. [1, Lemma 5.4]) a sequence $\left(\bar{\xi}_{h}\right)_{h}$ and a constant $C=C(D)$ such that

(i) $\bar{\xi}_{h} \in H_{0}^{1}(D ;[0,1]), \bar{\xi}_{h}=\chi_{D}$ on $\{x \in D: \operatorname{dist}(x, \partial D) \geq h\}$,

(ii) it holds $\int_{D} \nabla \phi \cdot \nabla \bar{\xi}_{h} \mathrm{~d} x \geq 0$ for all $\phi \in H_{0}^{1}(D ;[0, \infty))$,

(iii) supp $\nabla \bar{\xi}_{h} \subset\{x \in D$ : $\operatorname{dist}(x, \partial D)<h\}$, and we have the bounds

$$
C^{-1} \leq \int_{D}\left|\nabla \bar{\xi}_{h}\right| \mathrm{d} x \leq C, \quad \int_{D}\left|\nabla \bar{\xi}_{h}\right|^{2} \mathrm{~d} x \leq C h^{-1} .
$$


We then fix once and for all a scale $h \in\left(0, s_{c}\right)$, and set $\bar{\xi}:=\bar{\xi}_{h}$. Note that $\bar{\xi} \equiv 1$ on $K$ by the choice of $s_{c}$ and $h$. For purely technical reasons, we actually consider in the following a mollified version of $\bar{\xi}$. Let $\bar{\xi}_{l}:=\gamma_{l} * \bar{\xi}$ for $l>0$.

Let now $y \in \mathbf{R}^{d}$ and $s \in\left(0, T^{*}\right)$ be fixed. We then define the test function

$$
\xi(x, t, y, s):=\rho_{\tau}(t-s) \varphi\left(\frac{t+s}{2}\right) \gamma_{\theta}(x-y) \bar{\xi}_{l}\left(\frac{x+y}{2}\right), \quad(x, t) \in \mathbf{R}^{d} \times\left(0, T^{*}\right) .
$$

For this to be an admissible choice in (48) we need to restrict the range of the various parameters. Assuming that

$$
2 \tau<\min \left\{\inf \operatorname{supp} \varphi, T^{*}-\sup \operatorname{supp} \varphi\right\} \text { and } \theta \vee l<\frac{s_{c}}{4}
$$

we observe $\xi(\cdot, \cdot, y, s) \in C_{\mathrm{cpt}}^{\infty}\left(\mathbf{R}^{d} \times\left(0, T^{*}\right) ;[0, \infty)\right)$ and is thus admissible for (48). Moreover, for every $x \in \mathbf{R}^{d}$ and $t \in\left(0, T^{*}\right)$ the test function

$$
\tilde{\xi}(y, s, x, t):=\xi(x, t, y, s), \quad(y, s) \in \mathbf{R}^{d} \times\left(0, T^{*}\right)
$$

then also represents an admissible choice for (33) under the same restrictions (52) on the parameters. We have everything in place to merge (48) and (33).

Testing (33) with the admissible test functions $\xi(\cdot, \cdot, y, s)$ from (51) for every $(y, s) \in \mathbf{R}^{d} \times\left(0, T^{*}\right)$, integrating over $(y, s) \in \mathbf{R}^{d} \times\left(0, T^{*}\right)$, then repeating everything with (49) based on the admissible test functions $\tilde{\xi}(\cdot, \cdot, x, t)$ from $(53)$ for every $(x, t) \in$ $\mathbf{R}^{d} \times\left(0, T^{*}\right)$, and finally summing the two resulting inequalities (using in particular that $\eta_{\delta}$ is even) yields an estimate of the form

$$
\mathbf{E} R_{\mathrm{dt}} \leq \mathbf{E} R_{\text {porMed }}+\mathbf{E} R_{\mathrm{corr}}+\mathbf{E} R_{\text {noise }}+\mathbf{E} R_{\text {error }}
$$

for all $(\tau, \theta, l)$ subject to (52), all $\delta>0$ and all $\varepsilon, \hat{\varepsilon} \leq \frac{\kappa}{2}$. Here, we introduced for convenience the abbreviations

$$
\begin{aligned}
R_{\mathrm{dt}}:= & -\int_{0}^{T^{*}} \int_{\mathbf{R}^{d}} \int_{0}^{T^{*}} \int_{\mathbf{R}^{d}} \eta_{\delta}\left(v_{\varepsilon}(x, t)-v_{\hat{\varepsilon}}(y, s)\right)\left(\partial_{t}+\partial_{s}\right) \xi(x, t, y, s) \mathrm{d} y \mathrm{~d} s \mathrm{~d} x \mathrm{~d} t, \\
R_{\text {porMed }}:= & -\int_{0}^{T^{*}} \int_{\mathbf{R}^{d}} \int_{0}^{T^{*}} \int_{\mathbf{R}^{d}}\left|\nabla v_{\varepsilon}^{m}(x, t)\right|^{2}\left(\eta_{\delta}\right)^{\prime \prime}\left(v_{\varepsilon}^{m}(x, t)-v_{\hat{\varepsilon}}^{m}(y, s)\right) \\
& \times \xi(x, t, y, s) \mathrm{d} x \mathrm{~d} t \mathrm{~d} y \mathrm{~d} s \\
& +\int_{0}^{T^{*}} \int_{\mathbf{R}^{d}} \int_{0}^{T^{*}} \int_{\mathbf{R}^{d}} \eta_{\delta}\left(v_{\varepsilon}^{m}(x, t)-v_{\hat{\varepsilon}}^{m}(y, s)\right) \Delta_{x} \xi(x, t, y, s) \mathrm{d} x \mathrm{~d} t \mathrm{~d} y \mathrm{~d} s \\
& -\int_{0}^{T^{*}} \int_{\mathbf{R}^{d}} \int_{0}^{T^{*}} \int_{\mathbf{R}^{d}}\left|\nabla v_{\hat{\varepsilon}}^{m}(y, s)\right|^{2}\left(\eta_{\delta}\right)^{\prime \prime}\left(v_{\varepsilon}^{m}(x, t)-v_{\hat{\varepsilon}}^{m}(y, s)\right) \\
& +\int_{0}^{T^{*}} \int_{\mathbf{R}^{d}} \int_{0}^{T^{*}} \int_{\mathbf{R}^{d}} \eta_{\delta}\left(v_{\varepsilon}^{m}(x, t)-v_{\hat{\varepsilon}}^{m}(y, s)\right) \Delta_{y} \xi(x, t, y, s) \mathrm{d} y \mathrm{~d} s \mathrm{~d} x \mathrm{~d} t,
\end{aligned}
$$




$$
\begin{aligned}
R_{\text {corr }}:= & -\int_{0}^{T^{*}} \int_{\mathbf{R}^{d}} \int_{0}^{T^{*}} \int_{\mathbf{R}^{d}} \frac{1}{2} v^{2}\left|\nabla v_{\varepsilon}(x, t)\right|^{2}\left(\eta_{\delta}\right)^{\prime \prime}\left(v_{\varepsilon}(x, t)-v_{\hat{\varepsilon}}(y, s)\right) \\
& \times \xi(x, t, y, s) \mathrm{d} x \mathrm{~d} t \mathrm{~d} y \mathrm{~d} s \\
& +\int_{0}^{T^{*}} \int_{\mathbf{R}^{d}} \int_{0}^{T^{*}} \int_{\mathbf{R}^{d}} \frac{1}{2} v^{2} \eta_{\delta}\left(v_{\varepsilon}(x, t)-v_{\hat{\varepsilon}}(y, s)\right) \Delta_{x} \xi(x, t, y, s) \mathrm{d} x \mathrm{~d} t \mathrm{~d} y \mathrm{~d} s \\
& -\int_{0}^{T^{*}} \int_{\mathbf{R}^{d}} \int_{0}^{T^{*}} \int_{\mathbf{R}^{d}} \frac{1}{2} v^{2}\left|\nabla v_{\hat{\varepsilon}}(y, s)\right|^{2}\left(\eta_{\delta}\right)^{\prime \prime}\left(v_{\varepsilon}(x, t)-v_{\hat{\varepsilon}}(y, s)\right) \\
& +\int_{0}^{T^{*}} \int_{\mathbf{R}^{d}} \int_{0}^{T^{*}} \int_{\mathbf{R}^{d}} \frac{1}{2} v^{2} \eta_{\delta}\left(v_{\varepsilon}(x, t)-v_{\hat{\varepsilon}}(y, s)\right) \Delta_{y} \xi(x, t, y) \mathrm{d} y \mathrm{~d} s \mathrm{~d} x \mathrm{~d} t \\
R_{\text {noise }}:= & -\left.\int_{0}^{T^{*}} \int_{\mathbf{R}^{d}} \int_{0}^{T^{*}} \int_{\mathbf{R}^{d}} v \eta_{\delta}\left(v_{\varepsilon}(x, t)-\hat{z}\right) \nabla_{x} \xi(x, t, y, s) \mathrm{d} x \mathrm{~d} B_{t}\right|_{\hat{z}=v_{\hat{\varepsilon}}(y, s)} \mathrm{d} y \mathrm{~d} t, \\
& -\left.\int_{0}^{T^{*}} \int_{\mathbf{R}^{d}} \int_{0}^{T^{*}} \int_{\mathbf{R}^{d}} v \eta_{\delta}\left(z-v_{\hat{\varepsilon}}(y, s)\right) \nabla_{y} \xi(x, t, y, s) \mathrm{d} y \mathrm{~d} B_{s}\right|_{z=v_{\varepsilon}(x, t)} \mathrm{d} x \mathrm{~d} t,
\end{aligned}
$$

as well as

$$
\begin{aligned}
R_{\text {error }:=} & \int_{0}^{T^{*}} \int_{\mathbf{R}^{d}} \int_{0}^{T^{*}} \int_{\mathbf{R}^{d}}\left\{\left(\eta_{\delta}\right)^{\prime}\left(v_{\varepsilon}(x, t)-v_{\hat{\varepsilon}}(y, s)\right)-\left(\eta_{\delta}\right)^{\prime}\left(v_{\varepsilon}^{m}(x, t)-v_{\hat{\varepsilon}}^{m}(y, s)\right)\right\} \\
& \times\left(\Delta_{x} v_{\varepsilon}^{m}\right)(x, t) \xi(x, t, y, s) \mathrm{d} x \mathrm{~d} t \mathrm{~d} y \mathrm{~d} s \\
& +\int_{0}^{T^{*}} \int_{\mathbf{R}^{d}} \int_{0}^{T^{*}} \int_{\mathbf{R}^{d}}\left\{\left(\eta_{\delta}\right)^{\prime}\left(v_{\hat{\varepsilon}}(y, s)-v_{\varepsilon}(x, t)\right)-\left(\eta_{\delta}\right)^{\prime}\left(v_{\hat{\varepsilon}}^{m}(y, s)-v_{\varepsilon}^{m}(x, t)\right)\right\} \\
& \times\left(\Delta_{y} v_{\hat{\varepsilon}}^{m}\right)(y, s) \tilde{\xi}(y, s, x, t) \mathrm{d} y \mathrm{~d} s \mathrm{~d} x \mathrm{~d} t \\
& -\int_{0}^{T^{*}} \int_{\mathbf{R}^{d}} \int_{0}^{T^{*}} \int_{\mathbf{R}^{d}} m\left(\left(u_{\varepsilon}^{\leftarrow}\right)^{m-1}-v_{\varepsilon}^{m-1}\right)(x, t) \nabla_{x} \xi(x, t, y, s) \\
& -\int_{0}^{T^{*}} \int_{\mathbf{R}^{d}} \int_{0}^{T^{*}} \int_{\mathbf{R}^{d}} m\left(\left(u_{\hat{\varepsilon}} v_{\varepsilon}(x, t)\left(\eta_{\delta}\right)^{\prime}\left(v_{\varepsilon}(x, t)-v_{\hat{\varepsilon}}(y, s)\right) \mathrm{d} x \mathrm{~d} t \mathrm{~d} y \mathrm{~d} s\right.\right. \\
& \left.\cdot \nabla v_{\hat{\varepsilon}}^{m-1}\right)(y, s)\left(\eta_{\delta}\right)^{\prime}\left(v_{\hat{\varepsilon}}(y, s)-v_{\mathcal{\varepsilon}}(x, t)\right) \mathrm{d} y \mathrm{~d} s \mathrm{~d} x \mathrm{~d} t .
\end{aligned}
$$

Before we move on with removing the doubling in the time variable by studying the limit $\tau \rightarrow 0$ let us first perform some computations on the non-linear diffusion term $R_{\text {porMed }}$ and the correction term $R_{\text {corr }}$. Exploiting that $\left(\eta_{\delta}\right)^{\prime \prime} \geq 0$ and $\xi \geq 0$, completing the square $\left|\nabla v_{\varepsilon}^{m}\right|^{2}+\left|\nabla v_{\hat{\varepsilon}}^{m}\right|^{2}=\left|\nabla v_{\varepsilon}^{m}-\nabla v_{\hat{\varepsilon}}^{m}\right|^{2}+2 \nabla v_{\varepsilon}^{m} \cdot \nabla v_{\hat{\varepsilon}}^{m}$ and integrating by parts entails that

$$
\begin{aligned}
& -\int_{\mathbf{R}^{d}} \int_{\mathbf{R}^{d}}\left|\nabla v_{\varepsilon}^{m}(x, t)\right|^{2}\left(\eta_{\delta}\right)^{\prime \prime}\left(v_{\varepsilon}^{m}(x, t)-v_{\hat{\varepsilon}}^{m}(y, s)\right) \xi \mathrm{d} x \mathrm{~d} y \\
& \quad-\int_{\mathbf{R}^{d}} \int_{\mathbf{R}^{d}}\left|\nabla v_{\hat{\varepsilon}}^{m}(y, s)\right|^{2}\left(\eta_{\delta}\right)^{\prime \prime}\left(v_{\varepsilon}^{m}(x, t)-v_{\hat{\varepsilon}}^{m}(y, s)\right) \xi \mathrm{d} x \mathrm{~d} y
\end{aligned}
$$




$$
\begin{aligned}
& \leq \int_{\mathbf{R}^{d}} \int_{\mathbf{R}^{d}} 2 \xi\left(\nabla_{y} \cdot \nabla_{x}\right) \eta_{\delta}\left(v_{\varepsilon}^{m}(x, t)-v_{\hat{\varepsilon}}^{m}(y, s)\right) \mathrm{d} x \mathrm{~d} y \\
& =\int_{\mathbf{R}^{d}} \int_{\mathbf{R}^{d}} \eta_{\delta}\left(v_{\varepsilon}^{m}(x, t)-v_{\hat{\varepsilon}}^{m}(y, s)\right) 2\left(\nabla_{x} \cdot \nabla_{y}\right) \xi \mathrm{d} x \mathrm{~d} y .
\end{aligned}
$$

Since $\left(\Delta_{x}+\Delta_{y}\right)\left(\gamma_{\theta}(x-y) \bar{\xi}_{l}\left(\frac{x+y}{2}\right)\right)=\gamma_{\theta}(x-y) \frac{1}{2} \Delta \bar{\xi}_{l}\left(\frac{x+y}{2}\right)+\bar{\xi}_{l}\left(\frac{x+y}{2}\right) 2 \Delta \gamma_{\theta}(x-y)$ and $\left(2 \nabla_{x} \cdot \nabla_{y}\right)\left(\gamma_{\theta}(x-y) \bar{\xi}_{l}\left(\frac{x+y}{2}\right)\right)=\gamma_{\theta}(x-y) \frac{1}{2} \Delta \bar{\xi}_{l}\left(\frac{x+y}{2}\right)-\bar{\xi}_{l}\left(\frac{x+y}{2}\right) 2 \Delta \gamma_{\theta}(x-y)$, we thus obtain the estimate

$$
\begin{aligned}
R_{\text {porMed }} \leq \int_{0}^{T^{*}} \int_{\mathbf{R}^{d}} \int_{0}^{T^{*}} \int_{\mathbf{R}^{d}} & \eta_{\delta}\left(v_{\varepsilon}^{m}(x, t)-v_{\hat{\varepsilon}}^{m}(y, s)\right) \\
& \times \rho_{\tau}(t-s) \varphi\left(\frac{t+s}{2}\right) \gamma_{\theta}(x-y) \Delta \bar{\xi}_{l}\left(\frac{x+y}{2}\right) \mathrm{d} x \mathrm{~d} t \mathrm{~d} y \mathrm{~d} s \\
= & : R_{\text {porMed }}^{(1)} .
\end{aligned}
$$

The idea eventually is — after letting $\tau \rightarrow 0, \delta \rightarrow 0$ and removing the doubling in the spatial variables (the latter by fine-tuning the scales $\theta>0$ and $l>0$ as suitably chosen powers of $\varepsilon \vee \hat{\varepsilon}$ ) — to integrate by parts and to use the sign in condition $i i$ ) for the spatial test function $\bar{\xi}$. We will make this precise together with all the required error estimates in a later stage of the proof. For the moment, we only wish to mention that because of the convexity of $\eta_{\delta}$ and $\xi \geq 0$ it holds

$$
\begin{aligned}
R_{\text {corr }} & \leq \int_{0}^{T^{*}} \int_{\mathbf{R}^{d}} \int_{0}^{T^{*}} \int_{\mathbf{R}^{d}} \frac{1}{2} v^{2} \eta_{\delta}\left(v_{\varepsilon}(x, t)-v_{\hat{\varepsilon}}(y, s)\right)\left(\Delta_{x}+\Delta_{y}\right) \xi(x, t, y, s) \mathrm{d} x \mathrm{~d} t \mathrm{~d} y \mathrm{~d} s \\
& =: R_{\mathrm{corr}}^{(1)} .
\end{aligned}
$$

The task therefore reduces to post-process the bound

$$
\mathbf{E} R_{\mathrm{dt}} \leq \mathbf{E} R_{\text {porMed }}^{(1)}+\mathbf{E} R_{\text {corr }}^{(1)}+\mathbf{E} R_{\text {noise }}+\mathbf{E} R_{\text {error }}
$$

with the remaining three terms left unchanged from the estimate (54). In a first step we aim to remove the doubling in the time variable by letting $\tau \rightarrow 0$.

Lemma 9 Let the assumptions and notation of Sect. 4.2 until this point be in place. Define the quantities

$$
\begin{aligned}
R_{\mathrm{dt}}^{(1)} & :=-\int_{0}^{T^{*}} \int_{\mathbf{R}^{d}} \int_{\mathbf{R}^{d}} \eta_{\delta}\left(v_{\varepsilon}(x, t)-v_{\hat{\varepsilon}}(y, t)\right) \gamma_{\theta}(x-y) \bar{\xi}_{l}\left(\frac{x+y}{2}\right) \frac{\mathrm{d}}{\mathrm{d} t} \varphi(t) \mathrm{d} y \mathrm{~d} x \mathrm{~d} t, \\
R_{\mathrm{porMed}}^{(2)} & :=\int_{0}^{T^{*}} \int_{\mathbf{R}^{d}} \int_{\mathbf{R}^{d}} \eta_{\delta}\left(v_{\varepsilon}^{m}(x, t)-v_{\hat{\varepsilon}}^{m}(y, t)\right) \varphi(t) \gamma_{\theta}(x-y) \Delta \bar{\xi}_{l}\left(\frac{x+y}{2}\right) \mathrm{d} y \mathrm{~d} x \mathrm{~d} t, \\
R_{\mathrm{corr}}^{(2)} & :=\int_{0}^{T^{*}} \int_{\mathbf{R}^{d}} \int_{\mathbf{R}^{d}} \frac{1}{2} v^{2} \eta_{\delta}\left(v_{\varepsilon}(x, t)-v_{\hat{\varepsilon}}(y, t)\right) \varphi(t) \gamma_{\theta}(x-y) \Delta \bar{\xi}_{l}\left(\frac{x+y}{2}\right) \mathrm{d} y \mathrm{~d} x \mathrm{~d} t,
\end{aligned}
$$




$$
\begin{aligned}
& R_{\mathrm{error}}^{(1)}:= \int_{0}^{T^{*}} \int_{\mathbf{R}^{d}} \int_{\mathbf{R}^{d}}\left\{\left(\eta_{\delta}\right)^{\prime}\left(v_{\varepsilon}(x, t)-v_{\hat{\varepsilon}}(y, t)\right)-\left(\eta_{\delta}\right)^{\prime}\left(v_{\varepsilon}^{m}(x, t)-v_{\hat{\varepsilon}}^{m}(y, t)\right)\right\} \\
& \times\left(\Delta_{x} v_{\varepsilon}^{m}\right)(x, t) \gamma_{\theta}(x-y) \bar{\xi}_{l}\left(\frac{x+y}{2}\right) \varphi(t) \mathrm{d} y \mathrm{~d} x \mathrm{~d} t \\
&+\int_{0}^{T^{*}} \int_{\mathbf{R}^{d}} \int_{\mathbf{R}^{d}}\left\{\left(\eta_{\delta}\right)^{\prime}\left(v_{\hat{\varepsilon}}(y, t)-v_{\varepsilon}(x, t)\right)-\left(\eta_{\delta}\right)^{\prime}\left(v_{\hat{\varepsilon}}^{m}(y, t)-v_{\varepsilon}^{m}(x, t)\right)\right\} \\
& \times\left(\Delta_{y} v_{\hat{\varepsilon}}^{m}\right)(y, t) \gamma_{\theta}(x-y) \bar{\xi}_{l}\left(\frac{x+y}{2}\right) \varphi(t) \mathrm{d} y \mathrm{~d} x \mathrm{~d} t \\
&-\int_{0}^{T^{*}} \int_{\mathbf{R}^{d}} \int_{\mathbf{R}^{d}} m\left(\left(u_{\varepsilon}^{\leftarrow}\right)^{m-1}-v_{\varepsilon}^{m-1}\right)(x, t) \nabla_{x}\left(\gamma_{\theta}(x-y) \bar{\xi}_{l}\left(\frac{x+y}{2}\right)\right) \varphi(t) \\
&-\int_{0}^{T^{*}} \int_{\mathbf{R}^{d}} \int_{\mathbf{R}^{d}} m\left(\left(u_{\hat{\varepsilon}}^{\leftarrow}\right)^{m-1}-v_{\hat{\varepsilon}}^{m-1}\right)(y, t)\left(\eta_{\delta}\right)^{\prime}\left(v_{\varepsilon}(x, t)-v_{\hat{\varepsilon}}(y, t)\right) \mathrm{d} y \mathrm{~d} x \mathrm{~d} t \\
& \cdot \nabla v_{\hat{\varepsilon}}(y, t)\left(\eta_{\delta}(x)^{\prime}\left(v_{\hat{\varepsilon}}(y, t)-v_{\varepsilon}(x, t)\right) \mathrm{d} y \mathrm{~d} x \mathrm{~d} t .\right.
\end{aligned}
$$

Then the estimate

$$
\mathbf{E} R_{\mathrm{dt}}^{(1)} \leq \mathbf{E} R_{\text {porMed }}^{(2)}+\mathbf{E} R_{\mathrm{corr}}^{(2)}+\mathbf{E} R_{\mathrm{error}}^{(1)}
$$

holds true for all $(\theta, l)$ subject to (52), all $\delta>0$ and all $\varepsilon, \hat{\varepsilon} \leq \frac{\kappa}{2}$.

Proof It follows from (51) that $\left(\partial_{t}+\partial_{s}\right) \xi=\varphi^{\prime}\left(\frac{t+s}{2}\right) \rho_{\tau}(t-s) \gamma_{\theta}(x-y) \bar{\xi}_{l}\left(\frac{x+y}{2}\right)$. In particular, the singular terms as $\tau \rightarrow 0$ cancel. Hence, it follows by Lebesgue's dominated convergence based on the regularity and the bounds for the Wong-Zakai approximation $u_{\varepsilon}$ from Lemma 4 and definition (22) of the shifted densities $u_{\varepsilon}^{\leftarrow}$ that

$$
\mathbf{E} R_{\mathrm{dt}} \rightarrow \mathbf{E} R_{\mathrm{dt}}^{(1)} \text { as } \tau \rightarrow 0
$$

Relying again on Lebesgue's dominated convergence due to the regularity and the a priori estimates for the Wong-Zakai approximation $u_{\varepsilon}$ from Lemma 4 and the definition (22) of the shifted densities $u_{\varepsilon}^{\leftarrow}$, we may also easily pass to the limit $\tau \rightarrow 0$ in all the terms on the right hand side of (55) except for the noise term $R_{\text {noise }}$. More precisely, we obtain

$$
\begin{aligned}
\mathbf{E} R_{\text {porMed }}^{(1)} & \rightarrow \mathbf{E} R_{\text {porMed }}^{(2)} & & \text { as } \tau \rightarrow 0, \\
\mathbf{E} R_{\text {corr }}^{(1)} & \rightarrow \mathbf{E} \widetilde{R}_{\text {corr }}^{(2)}, & & \text { as } \tau \rightarrow 0, \\
\mathbf{E} R_{\text {error }} & \rightarrow \mathbf{E} R_{\text {error }}^{(1)} & & \text { as } \tau \rightarrow 0,
\end{aligned}
$$

with the shorthand 


$$
\begin{aligned}
\widetilde{R}_{\mathrm{corr}}^{(2)}:=\int_{0}^{T^{*}} \int_{\mathbf{R}^{d}} \int_{\mathbf{R}^{d}} & \frac{1}{2} v^{2} \eta_{\delta}\left(v_{\varepsilon}(x, t)-v_{\hat{\varepsilon}}(y, t)\right) \varphi(t) \\
& \times\left(\Delta_{x}+\Delta_{y}\right)\left(\gamma_{\theta}(x-y) \bar{\xi}_{l}\left(\frac{x+y}{2}\right)\right) \mathrm{d} y \mathrm{~d} x \mathrm{~d} t .
\end{aligned}
$$

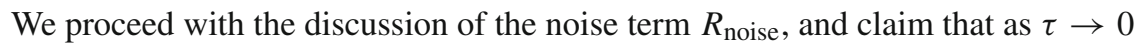

$$
\begin{aligned}
\mathbf{E} R_{\text {noise }} \rightarrow \int_{0}^{T^{*}} \int_{\mathbf{R}^{d}} \int_{\mathbf{R}^{d}} & \frac{1}{2} v^{2} \eta_{\delta}\left(v_{\varepsilon}(x, t)-v_{\hat{\varepsilon}}(y, t)\right) \varphi(t) \\
& \times 2\left(\nabla_{x} \cdot \nabla_{y}\right)\left(\gamma_{\theta}(x-y) \bar{\xi}_{l}\left(\frac{x+y}{2}\right)\right) \mathrm{d} y \mathrm{~d} x \mathrm{~d} t .
\end{aligned}
$$

Recall that $\left(\Delta_{x}+\Delta_{y}\right)\left(\gamma_{\theta}(x-y) \bar{\xi}_{l}\left(\frac{x+y}{2}\right)\right)=\gamma_{\theta}(x-y) \frac{1}{2} \Delta \bar{\xi}_{l}\left(\frac{x+y}{2}\right)+\bar{\xi}_{l}\left(\frac{x+y}{2}\right) 2 \Delta \gamma_{\theta}(x-y)$ and $2\left(\nabla_{x} \cdot \nabla_{y}\right)\left(\gamma_{\theta}(x-y) \bar{\xi}_{l}\left(\frac{x+y}{2}\right)\right)=\gamma_{\theta}(x-y) \frac{1}{2} \Delta \bar{\xi}_{l}\left(\frac{x+y}{2}\right)-\bar{\xi}_{l}\left(\frac{x+y}{2}\right) 2 \Delta \gamma_{\theta}(x-y)$. Hence, the combination of (63) and (65) yields

$$
\mathbf{E} R_{\text {corr }}^{(1)}+\mathbf{E} R_{\text {noise }} \rightarrow \mathbf{E} R_{\text {corr }}^{(2)}
$$

as $\tau \rightarrow 0$, which in view of (61), (62) and (64) entails the desired estimate (60). Hence, it remains to verify (65).

To this end, we again define for $\lambda>0$ the cut-off $\bar{\rho}_{\lambda}:=\frac{1}{\lambda} \bar{\rho}\left(\frac{\dot{\lambda}}{\lambda}\right)$ by means of a standard even cut-off function $\bar{\rho} \in C_{\mathrm{cpt}}^{\infty}((-1,1) ;[0, \infty))$ such that $\int_{\mathbf{R}} \bar{\rho}(r) \mathrm{d} r=1$. Furthermore, let $X(y, s ; \hat{z}):=-\int_{s}^{s+\tau} \int_{\mathbf{R}^{d}} v \eta_{\delta}\left(v_{\varepsilon}(x, t)-\hat{z}\right) \nabla_{x} \xi(x, t, y, s) \mathrm{d} x \mathrm{~d} B_{t}$ and $Y(x, t ; z):=-\int_{t-\tau}^{t} \int_{\mathbf{R}^{d}} v \eta_{\delta}\left(z-v_{\hat{\varepsilon}}(y, s)\right) \nabla_{y} \xi(x, t, y, s) \mathrm{d} y \mathrm{~d} B_{s}$. Hence, using that the mollifier $\rho$ is supported on the positive real axis, we may rewrite for all $\tau>0$ subject to (52)

$$
R_{\text {noise }}=\left.\int_{0}^{T^{*}-\tau} \int_{\mathbf{R}^{d}} X(y, s ; \hat{z})\right|_{\hat{z}=v_{\hat{\varepsilon}}(y, s)} \mathrm{d} y \mathrm{~d} s+\left.\int_{\tau}^{T^{*}} \int_{\mathbf{R}^{d}} Y(x, t ; z)\right|_{z=v_{\varepsilon}(x, t)} \mathrm{d} x \mathrm{~d} t .
$$

Based on the argument given in Step 4 of the proof of Lemma 8, it holds

$$
\begin{aligned}
\mathbf{E} R_{\text {noise }}= & \lim _{\lambda \rightarrow 0} \mathbf{E} \int_{0}^{T^{*}-\tau} \int_{\mathbf{R}^{d}} \int_{\mathbf{R}} \bar{\rho}_{\lambda}\left(v_{\hat{\varepsilon}}(y, s)-\hat{z}\right) X(y, s ; \hat{z}) \mathrm{d} \hat{z} \mathrm{~d} y \mathrm{~d} s \\
& +\lim _{\lambda \rightarrow 0} \mathbf{E} \int_{\tau}^{T^{*}} \int_{\mathbf{R}^{d}} \int_{\mathbf{R}} \bar{\rho}_{\lambda}\left(v_{\varepsilon}(x, t)-z\right) Y(x, t ; z) \mathrm{d} z \mathrm{~d} x \mathrm{~d} t \\
= & : \lim _{\lambda \rightarrow 0} \mathbf{E} R_{\text {noise }}^{(1), \lambda}+\lim _{\lambda \rightarrow 0} \mathbf{E} R_{\text {noise }}^{(2), \lambda} .
\end{aligned}
$$

Observe that $\mathbf{E} R_{\text {noise }}^{(1), \lambda}=0$ since the bounded random variable $v_{\hat{\varepsilon}}(y, s)$ is measurable with respect to $\mathcal{F}_{s}$. Similarly, due to the fact that $v_{\varepsilon}(x, t-\tau)$ is bounded and measurable 
with respect to $\mathcal{F}_{t-\tau}$ we may actually write for all $\lambda \in(0,1]$

$$
\mathbf{E} R_{\text {noise }}^{(2), \lambda}=\mathbf{E} \int_{\tau}^{T^{*}} \int_{\mathbf{R}^{d}} \int_{\mathbf{R}} Y(x, t ; z)\left\{\bar{\rho}_{\lambda}\left(v_{\varepsilon}(x, t)-z\right)-\bar{\rho}_{\lambda}\left(v_{\varepsilon}(x, t-\tau)-z\right)\right\} \mathrm{d} z \mathrm{~d} x \mathrm{~d} t .
$$

As a preparation for what follows, we present regularity estimates for $Y$. More precisely, we claim that for all $m, n \geq 1$ there exists a constant $C>0$ such that

$$
\left(\mathbf{E}\left\|\partial_{z}^{m} \nabla_{x}^{n} Y\right\|_{L_{x, t, z}^{\infty}}^{8}\left(\mathbf{R}^{d} \times[0, T] \times \mathbf{R}\right)\right)^{\frac{1}{8}} \leq C \tau^{-\frac{3}{4}}
$$

uniformly over all $\tau$ subject to (52). For a proof of (68), it suffices to show that

$$
\begin{gathered}
\left(\mathbf{E}\left\|\partial_{z}^{\widehat{m}} \nabla_{x}^{\widehat{n}} Y\right\|_{L_{t}^{8}\left([0, T] ; L_{x, z}^{8}\left(\mathbf{R}^{d} \times \mathbf{R}\right)\right)}^{8}\right)^{\frac{1}{8}} \leq C_{\widehat{m}, \widehat{n}} \tau^{-\frac{1}{2}}, \\
\left(\mathbf{E}\left\|\partial_{t} \partial_{z}^{\widehat{m}} \nabla_{x}^{\widehat{n}} Y\right\|_{L_{t}^{8}\left([0, T] ; L_{x, z}^{8}\left(\mathbf{R}^{d} \times \mathbf{R}\right)\right)}^{8}\right)^{\frac{1}{8}} \leq C_{\widehat{m}, \widehat{n}} \tau^{-\frac{3}{2}},
\end{gathered}
$$

uniformly over all $\tau$ subject to (52). Indeed, by passing through a fractional Sobolev space in the time variable we may interpolate between the previous two estimates to obtain $\mathbf{E}\left\|\partial_{z}^{\widehat{m}} \nabla_{x}^{\widehat{n}} Y\right\|_{W_{t}^{8, \delta}\left([0, T] ; L_{x, z}^{8}\left(\mathbf{R}^{d} \times \mathbf{R}\right)\right)}^{8} \leq C_{\delta, \widehat{m}, \widehat{n}} \tau^{8\left(-\delta-\frac{1}{2}\right)}$, uniformly over all $\tau$ subject to (52). In particular, fixing $\delta=\frac{1}{4}$ in combination with Sobolev embedding allows to deduce (68). For a proof of (69), we may appeal to the Burkholder-DavisGundy inequality and the maximum principle bound (15), which imply the desired estimate in form of (with the scaling in $\tau$ resulting from $\rho_{\tau}$ )

$$
\begin{aligned}
& \mathbf{E}\left\|\partial_{z}^{\widehat{m}} \nabla_{x}^{\widehat{n}} Y\right\|_{L_{t}^{8}\left([0, T] ; L_{x, z}^{8}\left(\mathbf{R}^{d} \times \mathbf{R}\right)\right)}^{8} \\
& \quad \leq\left.\left. C_{\widehat{m}} \tau^{-8} \int_{\tau}^{T^{*}} \int_{\mathbf{R}^{d}} \mathbf{E}\left|\int_{t-\tau}^{t} \int_{B_{\theta}(x)}\right| \nabla_{x}^{\widehat{n}} \nabla_{y}\left(\gamma_{\theta}(x-y) \bar{\xi}_{l}\left(\frac{x+y}{2}\right)\right)\right|^{2} \mathrm{~d} y \mathrm{~d} s\right|^{4} \mathrm{~d} x \mathrm{~d} t \\
& \quad \leq C_{\widehat{m}, \widehat{n}} \tau^{-4} .
\end{aligned}
$$

Similarly, one derives the estimate (70) with the scaling in $\tau$ stemming from $\partial_{t} \rho_{\tau}$.

Let now $f: \mathbf{R}^{d} \times\left(0, T^{*}\right) \rightarrow[0, \infty)$ be a smooth and compactly supported spacetime mollifier. It then follows from (39) that $f * u_{\varepsilon}^{\leftarrow}$ satisfies pointwise

$$
\mathrm{d}\left(f * u_{\varepsilon}^{\leftarrow}\right)_{t}=\left\{\Delta_{x}\left(f *\left(u_{\varepsilon}^{\leftarrow}\right)^{m}\right)+\frac{1}{2} v^{2} \Delta_{x}\left(f * u_{\varepsilon}^{\leftarrow}\right)\right\} \mathrm{d} t+v \nabla_{x}\left(f * u_{\varepsilon}^{\leftarrow}\right) \cdot \mathrm{d} B_{t}
$$

Applying Itô's formula to $F_{z} \circ\left(f * u_{\varepsilon}^{\leftarrow}\right), F_{z}:=\bar{\rho}_{\lambda}\left(\zeta_{\kappa}^{\varepsilon^{q}}(\cdot)-z\right) \in C^{\infty}(\mathbf{R})$, thus yields

$$
\begin{aligned}
& F_{z}\left(\left(f * u_{\varepsilon}^{\leftarrow}\right)(x, t)\right)-F_{z}\left(\left(f * u_{\varepsilon}^{\leftarrow}\right)(x, t-\tau)\right) \\
& \quad=\int_{t-\tau}^{t} F_{z}^{\prime}\left(\left(f * u_{\varepsilon}^{\leftarrow}\right)(x, \ell)\right)\left\{\Delta_{x}\left(f *\left(u_{\varepsilon}^{\leftarrow}\right)^{m}\right)+\frac{1}{2} v^{2} \Delta_{x}\left(f * u_{\varepsilon}^{\leftarrow}\right)\right\}(x, \ell) \mathrm{d} \ell
\end{aligned}
$$




$$
\begin{aligned}
& +\int_{t-\tau}^{t} \frac{1}{2} v^{2} F_{z}^{\prime \prime}\left(\left(f * u_{\varepsilon}^{\leftarrow}\right)(x, \ell)\right)\left|\nabla_{x}\left(f * u_{\varepsilon}^{\leftarrow}\right)\right|^{2}(x, \ell) \mathrm{d} \ell \\
& +\int_{t-\tau}^{t} v F_{z}^{\prime}\left(\left(f * u_{\varepsilon}^{\leftarrow}\right)(x, \ell)\right) \nabla_{x}\left(f * u_{\varepsilon}^{\leftarrow}\right)(x, \ell) \mathrm{d} B_{\ell}
\end{aligned}
$$

for all $x \in \mathbf{R}^{d}$, all $t \in\left(\tau, T^{*}\right)$ and all $z \in \mathbf{R}$. The left hand side term of (71) represents a proxy for $\bar{\rho}_{\lambda}\left(v_{\varepsilon}(x, t)-z\right)-\bar{\rho}_{\lambda}\left(v_{\varepsilon}(x, t-\tau)-z\right)$ in (67). Substituting this proxy into the right hand side term of (67), then inserting the identity (71), integrating by parts once in the terms with second-order spatial derivatives, and exploiting Itô's isometry for the third right hand side term of (71), we deduce that it holds

$$
\begin{aligned}
\mathbf{E} \int_{\tau}^{T^{*}} \int_{\mathbf{R}^{d}} \int_{\mathbf{R}} Y(x, t ; z)\left\{F_{z}\left(\left(f * u_{\varepsilon}^{\leftarrow}\right)(x, t)\right)-F_{z}\left(\left(f * u_{\varepsilon}^{\leftarrow}\right)(x, t-\tau)\right)\right\} \mathrm{d} z \mathrm{~d} x \mathrm{~d} t \\
=-\mathbf{E} \int_{\tau}^{T^{*}} \int_{\mathbf{R}^{d}} \int_{\mathbf{R}} \nabla_{x} Y(x, t ; z) \cdot \int_{t-\tau}^{t} F_{z}^{\prime}\left(\left(f * u_{\varepsilon}^{\leftarrow}\right)(x, \ell)\right) \\
\quad-\mathbf{E} \int_{\tau}^{T^{*}} \int_{\mathbf{R}^{d}} \int_{\mathbf{R}} Y(x, t ; z) \int_{t-\tau}^{t} F_{z}^{\prime \prime}\left(\left(f * u_{\varepsilon}^{\leftarrow}\right)(x, \ell)\right) \\
-\mathbf{E} \int_{\tau}^{T^{*}} \int_{\mathbf{R}^{d}} \int_{\mathbf{R}} \nabla_{x} Y(x, t ; z) \cdot \int_{t-\tau}^{t} F_{z}^{\prime}\left(\left(f * u_{\varepsilon}^{\leftarrow}\right)(x, \ell)\right) \mathrm{d} \ell \mathrm{d} z \mathrm{~d} x \mathrm{~d} t \\
-\mathbf{E} \int_{\tau}^{T^{*}} \int_{\mathbf{R}^{d}} \int_{\mathbf{R}} \int_{t-\tau}^{t} \int_{\mathbf{R}^{d}} v^{2} \eta_{\delta}\left(z-v_{\hat{\varepsilon}}(y, s)\right) \nabla_{y} \xi(x, t, y, s) \\
\cdot \nabla_{x}\left(F _ { z } \circ \left(f * v_{x}\left(\left.f u_{\varepsilon}^{\leftarrow}\right|^{2}\right)(x, \ell) \mathrm{d} \ell \mathrm{d} z \mathrm{~d} x \mathrm{~d} t\right.\right. \\
\end{aligned}
$$

Based on the regularity estimates for $Y$, the estimates for $u_{\varepsilon}$ from Lemma 4 , and the support properties of the test function $\xi$, we may let the mollifier $f$ run through a space-time Dirac sequence and pass to the limit. This updates (67) to

$$
\begin{array}{r}
\mathbf{E} R_{\text {noise }}^{(2), \lambda} \\
=-\mathbf{E} \int_{\tau}^{T^{*}} \int_{\mathbf{R}^{d}} \int_{\mathbf{R}} \partial_{z} \nabla_{x} Y(x, t ; z) \cdot \int_{t-\tau}^{t} \bar{\rho}_{\lambda}\left(v_{\varepsilon}(x, \ell)-z\right)\left(\zeta_{\kappa}^{\varepsilon^{q}}\right)^{\prime}\left(u_{\varepsilon}^{\leftarrow}(x, \ell)\right) \\
\times \nabla_{x}\left(u_{\varepsilon}^{\leftarrow}\right)^{m}(x, \ell) \mathrm{d} \ell \mathrm{d} z \mathrm{~d} x \mathrm{~d} t \\
-\mathbf{E} \int_{\tau}^{T^{*}} \int_{\mathbf{R}^{d}} \int_{\mathbf{R}} \partial_{z} \nabla_{x} Y(x, t ; z) \cdot \int_{t-\tau}^{t} \bar{\rho}_{\lambda}\left(v_{\varepsilon}(x, \ell)-z\right)\left(\zeta_{\kappa}^{\varepsilon^{q}}\right)^{\prime}\left(u_{\varepsilon}^{\leftarrow}(x, \ell)\right) \\
\times \frac{1}{2} v^{2} \nabla_{x} u_{\varepsilon}^{\leftarrow}(x, \ell) \mathrm{d} \ell \mathrm{d} z \mathrm{~d} x \mathrm{~d} t
\end{array}
$$




$$
\begin{gathered}
-\mathbf{E} \int_{\tau}^{T^{*}} \int_{\mathbf{R}^{d}} \int_{\mathbf{R}} \partial_{z} Y(x, t ; z) \int_{t-\tau}^{t} \bar{\rho}_{\lambda}\left(v_{\varepsilon}(x, \ell)-z\right)\left(\zeta_{\kappa}^{\varepsilon^{q}}\right)^{\prime \prime}\left(u_{\varepsilon}^{\leftarrow}(x, \ell)\right) \\
\times m\left(u_{\varepsilon}^{\leftarrow}\right)^{m-1}(x, \ell)\left|\nabla_{x} u_{\varepsilon}^{\leftarrow}\right|^{2}(x, \ell) \mathrm{d} \ell \mathrm{d} z \mathrm{~d} x \mathrm{~d} t \\
-\mathbf{E} \int_{\tau}^{T^{*}} \int_{\mathbf{R}^{d}} \int_{\mathbf{R}} \partial_{z}^{2} Y(x, t ; z) \int_{t-\tau}^{t} \bar{\rho}_{\lambda}\left(v_{\varepsilon}(x, \ell)-z\right)\left|\left(\zeta_{\kappa}^{\varepsilon^{q}}\right)^{\prime}\left(u_{\varepsilon}^{\leftarrow}(x, \ell)\right)\right|^{2} \\
\times m\left(u_{\varepsilon}^{\leftarrow}\right)^{m-1}(x, \ell)\left|\nabla_{x} u_{\varepsilon}^{\leftarrow}\right|^{2}(x, \ell) \mathrm{d} \ell \mathrm{d} z \mathrm{~d} x \mathrm{~d} t \\
-\mathbf{E} \int_{\tau}^{T^{*}} \int_{\mathbf{R}^{d}} \int_{\mathbf{R}} \int_{t-\tau}^{t} \int_{\mathbf{R}^{d}} v^{2} \eta_{\delta}\left(z-v_{\hat{\varepsilon}}(y, s)\right) \nabla_{y} \xi(x, t, y, s) \\
=: \mathbf{E} R_{\text {noise }}^{(3), \lambda}+\mathbf{E} R_{\text {noise }}^{(4), \lambda}+\mathbf{E} R_{\text {noise }}^{(5), \lambda}+\mathbf{E} R_{\text {noise }}^{(6), \lambda}+\mathbf{E} R_{\text {noise }}^{(7), \lambda},
\end{gathered}
$$

where we also integrated by parts in the $z$-variable to avoid derivatives of $\bar{\rho}_{\lambda}$ (which is required for $\lambda \rightarrow 0$ ).

The upshot of the argument is now the following. Based on the regularity estimates for $Y$, the estimates for $u_{\varepsilon}$ from Lemma 4 , and the support properties of the test function $\xi$, we claim that it holds

$$
\lim _{\tau \rightarrow 0} \lim _{\lambda \rightarrow 0} \sum_{i=3}^{6} \mathbf{E} R_{\text {noise }}^{(i), \lambda}=0
$$

We give details for the term $R_{\text {noise }}^{(5), \lambda}$; the other terms may be dealt with similarly. By Hölder's inequality with respect to the exponents $\left(q, \frac{q+1}{q}\right), q=8$, the bound $\int_{\tau}^{T^{*}} \int_{t-\tau}^{t}|g(\ell)| \mathrm{d} \ell \mathrm{d} t \leq \tau \int_{0}^{T^{*}}|g(t)| \mathrm{d} t$, and the estimate (15), we obtain $\mathbf{E}\left|R_{\text {noise }}^{(5), \lambda}\right|$

$$
\leq \tau\left(\mathbf{E}\left\|\partial_{z} Y\right\|_{L_{x, t, z}^{\infty}\left(\mathbf{R}^{d} \times[0, T] \times \mathbf{R}\right)}^{q}\right)^{\frac{1}{q}}\left(\left.\left.\mathbf{E}\left|\int_{0}^{T^{*}} \int_{\mathbf{R}^{d}} m\left(u_{\varepsilon}^{\leftarrow}\right)^{m-1}\right| \nabla u_{\varepsilon}^{\leftarrow}\right|^{2} \mathrm{~d} x \mathrm{~d} t\right|^{\frac{q+1}{q}}\right)^{\frac{q}{q+1}}
$$

uniformly over all $\tau$ subject to (52) and all $\lambda \in(0,1]$. By a change of variables to switch from $u_{\varepsilon}^{\leftarrow}$ to $u_{\varepsilon}$, the energy estimate (16), as well as the the regularity estimate (68) for $Y$, we may deduce from the previous display that $\mathbf{E}\left|R_{\text {noise }}^{(5), \lambda}\right| \leq C \tau^{\frac{1}{4}}$ uniformly over all $\tau$ subject to (52) and all $\lambda \in(0,1]$. Hence, we obtain as claimed $\lim _{\tau \rightarrow 0} \lim _{\lambda \rightarrow 0} \mathbf{E} R_{\text {noise }}^{(5), \lambda}=0$.

It remains to consider the term $R_{\text {noise }}^{(7), \lambda}$. However, after performing an integration by parts it directly follows (the limits $\tau \rightarrow 0$ and $\lambda \rightarrow 0$ are unproblematic by the support properties of $\xi$, and the regularity properties of $u_{\varepsilon}$ due to Lemma 4)

$$
\begin{aligned}
\lim _{\tau \rightarrow 0} \lim _{\lambda \rightarrow 0} \mathbf{E} R_{\text {noise }}^{(7), \lambda}=\int_{0}^{T^{*}} \int_{\mathbf{R}^{d}} \int_{\mathbf{R}^{d}} & \frac{1}{2} v^{2} \eta_{\delta}\left(v_{\varepsilon}(x, t)-v_{\hat{\varepsilon}}(y, t)\right) \varphi(t) \\
& \times 2\left(\nabla_{x} \cdot \nabla_{y}\right)\left(\gamma_{\theta}(x-y) \bar{\xi}_{l}\left(\frac{x+y}{2}\right)\right) \mathrm{d} y \mathrm{~d} x \mathrm{~d} t .
\end{aligned}
$$


The combination of (72)-(74) finally entails (65), which in turn concludes the proof of Lemma 9 as already argued above.

We continue with the proof of Proposition 5 taking care in the next step of the error terms. More precisely, we may derive the following bound.

Lemma 10 Let the assumptions and notation of Sect. 4.2 until this point be in place. In particular, recall the definition of the error term $R_{\mathrm{error}}^{(1)}$ from (59). We then have the estimate

$$
\mathbf{E} R_{\mathrm{error}}^{(1)} \leq C(\kappa) l^{-1} \theta^{-1}(\varepsilon \vee \hat{\varepsilon})^{q}+O_{\delta \rightarrow 0}(1)
$$

for all $(\theta, l)$ subject to (52), all $\delta>0$ and all $\varepsilon, \hat{\varepsilon} \leq \frac{\kappa}{2}$.

Proof We start estimating by Hölder's inequality

$$
\begin{aligned}
\mathbf{E} \mid \int_{0}^{T^{*}} \int_{\mathbf{R}^{d}} \int_{\mathbf{R}^{d}}\left(\Delta_{x} v_{\varepsilon}^{m}\right)(x, t) \gamma_{\theta}(x-y) \bar{\xi}_{l}\left(\frac{x+y}{2}\right) \varphi(t) \\
\quad \times\left\{\left(\eta_{\delta}\right)^{\prime}\left(v_{\varepsilon}(x, t)-v_{\hat{\varepsilon}}(y, t)\right)-\left(\eta_{\delta}\right)^{\prime}\left(v_{\varepsilon}^{m}(x, t)-v_{\hat{\varepsilon}}^{m}(y, t)\right)\right\} \mathrm{d} y \mathrm{~d} x \mathrm{~d} t \mid \\
\leq \quad\left(\mathbf{E} \int_{0}^{T^{*}} \int_{\mathbf{R}^{d}} \int_{\mathbf{R}^{d}}\left|\left(\eta_{\delta}\right)^{\prime}\left(v_{\varepsilon}(x, t)-v_{\hat{\varepsilon}}(y, t)\right)-\left(\eta_{\delta}\right)^{\prime}\left(v_{\varepsilon}^{m}(x, t)-v_{\hat{\varepsilon}}^{m}(y, t)\right)\right|^{2} \mathrm{~d} y \mathrm{~d} x \mathrm{~d} t\right)^{\frac{1}{2}} \\
\quad \times \frac{\|\varphi\|_{L^{\infty}\left(0, T^{*}\right)}}{(\inf \operatorname{supp} \varphi)^{\frac{1}{2}}}\left(\mathbf{E} \int_{0}^{T^{*}} \int_{\mathbf{R}^{d}} t\left|\left(\Delta_{x} v_{\varepsilon}^{m}\right)(x, t)\right|^{2} \mathrm{~d} x \mathrm{~d} t\right)^{\frac{1}{2}}\left\|\bar{\xi}_{l}\right\|_{L^{\infty}\left(\mathbf{R}^{d}\right)}\left\|\gamma_{\theta}\right\|_{L^{\infty}\left(\mathbf{R}^{d}\right)} .
\end{aligned}
$$

However, by the bounds (18) and (30), the convergence (31) and the fact that $\operatorname{sign}(a-b)=\operatorname{sign}\left(a^{m}-b^{m}\right)$ due to the monotonicity of $r \mapsto r^{m}$ we infer by an application of Lebesgue's dominated convergence theorem based on the regularity of the Wong-Zakai approximation $u_{\varepsilon}$ from Lemma 4 that the term on the right hand side of the latter bound vanishes as $\delta \rightarrow 0$.

Recall that $\kappa \leq v_{\varepsilon} \leq u_{\varepsilon}^{\leftarrow}$ on $\operatorname{supp} \nabla v_{\varepsilon}$. Hence, we can estimate by means of (21)

$$
\begin{aligned}
& \chi_{\operatorname{supp} \nabla v_{\varepsilon}}\left|\left(u_{\varepsilon}^{\leftarrow}\right)^{m-1}(x, t)-v_{\varepsilon}^{m-1}(x, t)\right| \\
& \quad \leq \chi_{\operatorname{supp} \nabla v_{\varepsilon}} \sup _{r \in\left[v_{\varepsilon}(x, t), u_{\varepsilon}^{\leftarrow}(x, t)\right]}(m-1) r^{m-2}\left|v_{\varepsilon}(x, t)-u_{\varepsilon}^{\leftarrow}(x, t)\right| \\
& \leq C(\kappa) \chi_{\operatorname{supp} \nabla v_{\varepsilon}} \varepsilon^{q} .
\end{aligned}
$$

We may then estimate using also (19), the definition (22) of the shifted densities $u_{\varepsilon}^{\leftarrow},\left\|\nabla \bar{\xi}_{l}\right\|_{L^{\infty}\left(\mathbf{R}^{d}\right)} \leq\left\|\nabla \gamma_{l}\right\|_{L^{1}\left(\mathbf{R}^{d}\right)}\|\bar{\xi}\|_{L^{\infty}\left(\mathbf{R}^{d}\right)} \leq C l^{-1}$ (which follows from Young's inequality and condition $i$ ) for the spatial cut-off $\bar{\xi}),\|\varphi\|_{L^{\infty}\left(0, T^{*}\right)} \leq 1$, a change of variables $x \mapsto x-v\left(B_{t}-B_{t}^{\varepsilon}\right)$, the fact that $u_{\varepsilon}^{\leftarrow} \geq \kappa$ on supp $\nabla v_{\varepsilon}$, and finally the a priori estimate (16) 


$$
\begin{aligned}
& \mathbf{E} \mid \int_{0}^{T^{*}} \int_{\mathbf{R}^{d}} \int_{\mathbf{R}^{d}}\left(\left(u_{\varepsilon}^{\leftarrow}\right)^{m-1}(x, t)-v_{\varepsilon}^{m-1}(x, t)\right) \\
& \quad \times \nabla v_{\varepsilon}(x, t) \cdot \nabla_{x}\left(\gamma_{\theta}(x-y) \bar{\xi}_{l}\left(\frac{x+y}{2}\right)\right) \varphi(t) \mathrm{d} y \mathrm{~d} x \mathrm{~d} t \mid \\
& \leq C l^{-1} \theta^{-1} \mathbf{E} \int_{0}^{T^{*}} \int_{\mathbf{R}^{d}} \chi_{\operatorname{supp} \nabla v_{\varepsilon}}\left|\left(u_{\varepsilon}^{\leftarrow}\right)^{m-1}(x, t)-v_{\varepsilon}^{m-1}(x, t)\right|\left|\nabla u_{\varepsilon}^{\leftarrow}(x, t)\right| \mathrm{d} x \mathrm{~d} t \\
& \leq C(\kappa) l^{-1} \theta^{-1} \varepsilon^{q}\left(\mathbf{E} \int_{0}^{T^{*}} \int_{D} m u_{\varepsilon}^{m-1}(x, t)\left|\nabla u_{\varepsilon}(x, t)\right|^{2} \mathrm{~d} x \mathrm{~d} t\right)^{\frac{1}{2}} \\
& \leq C(\kappa) l^{-1} \theta^{-1} \varepsilon^{q} .
\end{aligned}
$$

Since the other two terms of $R_{\text {error }}^{(1)}$ can be treated along the same lines, we obtain in total the asserted estimate (75). This concludes the proof of Lemma 10.

We continue with the proof of Proposition 5. We have by now everything in place to let $\delta \rightarrow 0$ in (60). A straightforward application of Lebesgue's dominated convergence theorem based on the convergence in (31) and the regularity and bounds for the WongZakai approximations $u_{\varepsilon}$ from Lemma 4 shows that by letting $\delta \rightarrow 0$ in (60) and using (75) it holds

$$
\mathbf{E} R_{\mathrm{dt}}^{(2)} \leq \mathbf{E} R_{\text {porMed }}^{(3)}+\mathbf{E} R_{\mathrm{corr}}^{(3)}+C(\kappa) l^{-1} \theta^{-1}(\varepsilon \vee \hat{\varepsilon})^{q}
$$

for all $(\theta, l)$ subject to (52), and all $\varepsilon, \hat{\varepsilon} \leq \frac{\kappa}{2}$. The updated terms in this inequality are given by

$$
\begin{aligned}
R_{\mathrm{dt}}^{(2)} & :=-\int_{0}^{T^{*}} \int_{\mathbf{R}^{d}} \int_{\mathbf{R}^{d}}\left|v_{\varepsilon}(x, t)-v_{\hat{\varepsilon}}(y, t)\right| \gamma_{\theta}(x-y) \bar{\xi}_{l}\left(\frac{x+y}{2}\right) \frac{\mathrm{d}}{\mathrm{d} t} \varphi(t) \mathrm{d} y \mathrm{~d} x \mathrm{~d} t, \\
R_{\mathrm{porMed}}^{(3)} & :=\int_{0}^{T^{*}} \int_{\mathbf{R}^{d}} \int_{\mathbf{R}^{d}}\left|v_{\varepsilon}^{m}(x, t)-v_{\hat{\varepsilon}}^{m}(y, t)\right| \varphi(t) \gamma_{\theta}(x-y) \Delta \bar{\xi}_{l}\left(\frac{x+y}{2}\right) \mathrm{d} y \mathrm{~d} x \mathrm{~d} t, \\
R_{\mathrm{corr}}^{(3)} & :=\int_{0}^{T^{*}} \int_{\mathbf{R}^{d}} \int_{\mathbf{R}^{d}} \frac{1}{2} v^{2}\left|v_{\varepsilon}(x, t)-v_{\hat{\varepsilon}}(y, t)\right| \varphi(t) \gamma_{\theta}(x-y) \Delta \bar{\xi}_{l}\left(\frac{x+y}{2}\right) \mathrm{d} y \mathrm{~d} x \mathrm{~d} t,
\end{aligned}
$$

respectively. We may proceed with the next step which consists of removing the doubling in the spatial variables. More precisely, the following holds true.

Lemma 11 Let the assumptions and notation of Sect. 4.2 until this point be in place. Define the quantities

$$
\begin{aligned}
R_{\mathrm{dt}}^{(3)} & :=-\int_{0}^{T^{*}} \int_{\mathbf{R}^{d}} \int_{\mathbf{R}^{d}}\left|v_{\varepsilon}(x, t)-v_{\hat{\varepsilon}}(y, t)\right| \gamma_{\theta}(x-y) \bar{\xi}_{l}(x) \frac{\mathrm{d}}{\mathrm{d} t} \varphi(t) \mathrm{d} y \mathrm{~d} x \mathrm{~d} t, \\
R_{\text {porMed }}^{(5)} & :=\int_{0}^{T^{*}} \varphi(t) \int_{\mathbf{R}^{d}}\left|v_{\varepsilon}^{m}(x, t)-v_{\hat{\varepsilon}}^{m}(x, t)\right| \Delta \bar{\xi}_{l}(x) \mathrm{d} x \mathrm{~d} t, \\
R_{\mathrm{corr}}^{(5)} & :=\int_{0}^{T^{*}} \varphi(t) \int_{\mathbf{R}^{d}} \frac{1}{2} v^{2}\left|v_{\varepsilon}(x, t)-v_{\hat{\varepsilon}}(x, t)\right| \Delta \bar{\xi}_{l}(x) \mathrm{d} x \mathrm{~d} t .
\end{aligned}
$$


We then have the estimate

$$
\mathbf{E} R_{\mathrm{dt}}^{(3)} \leq \mathbf{E} R_{\text {porMed }}^{(5)}+\mathbf{E} R_{\text {corr }}^{(5)}+C(\kappa) l^{-1} \theta^{-1}(\varepsilon \vee \hat{\varepsilon})^{q}+C(\kappa) l^{-3} \theta
$$

for all $\varphi \in C_{\mathrm{cpt}}^{\infty}\left(\left(0, T^{*}\right) ;[0,1]\right)$ with $\|\varphi\|_{W^{1,1}\left(0, T^{*}\right)} \leq \bar{C}$, all $(\theta, l)$ subject to $(52)$ and all $\varepsilon, \hat{\varepsilon} \leq \frac{\kappa}{2}$.

Proof By a straightforward estimate, we may replace in all three terms $\bar{\xi}_{l}\left(\frac{-y}{2}\right)$ by $\bar{\xi}_{l}(\cdot)$ and therefore update the estimate (76) to

$$
\mathbf{E} R_{\mathrm{dt}}^{(3)} \leq \mathbf{E} R_{\text {porMed }}^{(4)}+\mathbf{E} R_{\text {corr }}^{(4)}+C(\kappa) l^{-1} \theta^{-1}(\varepsilon \vee \hat{\varepsilon})^{q}+C l^{-3} \theta\|\varphi\|_{W^{1,1,}\left(0, T^{*}\right)}
$$

for all $(\theta, l)$ subject to (52) and all $\varepsilon, \hat{\varepsilon} \leq \frac{\kappa}{2}$, where

$$
\begin{aligned}
R_{\mathrm{porMed}}^{(4)} & :=\int_{0}^{T^{*}} \int_{\mathbf{R}^{d}} \int_{\mathbf{R}^{d}}\left|v_{\varepsilon}^{m}(x, t)-v_{\hat{\varepsilon}}^{m}(y, t)\right| \varphi(t) \gamma_{\theta}(x-y) \Delta \bar{\xi}_{l}(x) \mathrm{d} y \mathrm{~d} x \mathrm{~d} t, \\
R_{\mathrm{corr}}^{(4)} & :=\int_{0}^{T^{*}} \int_{\mathbf{R}^{d}} \int_{\mathbf{R}^{d}} \frac{1}{2} v^{2}\left|v_{\varepsilon}(x, t)-v_{\hat{\varepsilon}}(y, t)\right| \varphi(t) \gamma_{\theta}(x-y) \Delta \bar{\xi}_{l}(x) \mathrm{d} y \mathrm{~d} x \mathrm{~d} t,
\end{aligned}
$$

respectively. We estimate using $\left\|\Delta \bar{\xi}_{l}\right\|_{L^{\infty}\left(\mathbf{R}^{d}\right)} \leq\left\|\nabla^{2} \gamma_{l}\right\|_{L^{1}\left(\mathbf{R}^{d}\right)}\|\bar{\xi}\|_{L^{\infty}\left(\mathbf{R}^{d}\right)} \leq C l^{-2}$ (which follows from Young's inequality and condition $i$ ) for the spatial cut-off $\bar{\xi}$ ), a change of variables $y \mapsto y+x, u_{\varepsilon}^{\leftarrow} \geq \kappa$ on supp $\nabla v_{\varepsilon}$, and the bounds (19) resp. (16)

$$
\begin{aligned}
\mid \mathbf{E} & R_{\text {corr }}^{(4)}-\mathbf{E} \int_{0}^{T^{*}} \int_{\mathbf{R}^{d}} \frac{1}{2} v^{2}\left|v_{\varepsilon}(x, t)-v_{\hat{\varepsilon}}(x, t)\right| \varphi(t) \Delta \bar{\xi}_{l}(x) \mathrm{d} x \mathrm{~d} t \mid \\
& \leq C \mathbf{E} \int_{0}^{T^{*}} \int_{\mathbf{R}^{d}} \int_{\mathbf{R}^{d}}\left|v_{\hat{\varepsilon}}(y, t)-v_{\hat{\varepsilon}}(x, t)\right| \gamma_{\theta}(x-y)\left|\Delta \bar{\xi}_{l}(x)\right| \varphi(t) \mathrm{d} y \mathrm{~d} x \mathrm{~d} t \\
& \leq C l^{-2} \theta \mathbf{E} \int_{0}^{T^{*}} \int_{\mathbf{R}^{d}} \int_{\mathbf{R}^{d}} \gamma_{\theta}(x-y) \int_{0}^{1}\left|\nabla v_{\hat{\varepsilon}}(r x+(1-r) y, t)\right| \mathrm{d} r \mathrm{~d} y \mathrm{~d} x \mathrm{~d} t \\
& \leq C(\kappa) l^{-2} \theta \mathbf{E} \int_{0}^{1} \int_{0}^{T^{*}} \int_{\mathbf{R}^{d}} \int_{\mathbf{R}^{d}} \gamma_{\theta}(y)\left(u_{\hat{\varepsilon}}^{\leftarrow}\right)^{\frac{m-1}{2}}(x-r y, t)\left|\nabla u_{\hat{\varepsilon}}^{\leftarrow}(x-r y, t)\right| \mathrm{d} x \mathrm{~d} y \mathrm{~d} t \mathrm{~d} r \\
& \leq C(\kappa) l^{-2} \theta\left(\mathbf{E} \int_{0}^{T^{*}} \int_{D} m u_{\hat{\varepsilon}}^{m-1}(x, t)\left|\nabla u_{\hat{\varepsilon}}(x, t)\right|^{2} \mathrm{~d} x \mathrm{~d} t\right)^{\frac{1}{2}} \\
& \leq C(\kappa) l^{-2} \theta
\end{aligned}
$$

for all $(\theta, l)$ subject to (52) and all $\varepsilon, \hat{\varepsilon} \leq \frac{\kappa}{2}$. Arguing along the same lines we also get the estimate

$$
\left|\mathbf{E} R_{\text {porMed }}^{(4)}-\mathbf{E} \int_{0}^{T^{*}} \int_{\mathbf{R}^{d}}\right| v_{\varepsilon}^{m}(x, t)-v_{\hat{\varepsilon}}^{m}(x, t)\left|\varphi(t) \Delta \bar{\xi}_{l}(x) \mathrm{d} x \mathrm{~d} t\right| \leq C(\kappa) l^{-2} \theta
$$

for all $(\theta, l)$ subject to (52) and all $\varepsilon, \hat{\varepsilon} \leq \frac{\kappa}{2}$. Summarizing we then obtain the desired inequality (77). This concludes the proof of Lemma 11. 
We continue with the proof of Proposition 5. The next step takes care of estimating the non-linear diffusion term and the correction term.

Lemma 12 Let the assumptions and notation of Sect. 4.2 until this point be in place. In particular, recall from the statement of Lemma 11 the definition of the quantities $R_{\text {porMed }}^{(5)}$ and $R_{\text {corr }}^{(5)}$ Then there exists some $l(\bar{\xi})>0$ small enough and some absolute constant $\bar{C}>0$ such that we have the estimate

$$
\mathbf{E} R_{\text {porMed }}^{(5)}+\mathbf{E} R_{\text {corr }}^{(5)} \leq \bar{C} \kappa+C(\kappa) l^{-2}(\varepsilon \vee \hat{\varepsilon})^{\alpha}
$$

for all $(\theta, l)$ subject to $(52)$ resp. $l<l(\bar{\xi})$, and all $\varepsilon, \hat{\varepsilon} \leq \frac{\kappa}{2}$.

Proof We first aim to replace $v_{\varepsilon}$ resp. $v_{\hat{\varepsilon}}$ by $\zeta_{\kappa}^{\varepsilon^{q}} \circ u_{\varepsilon}$ resp. $\zeta_{\kappa}^{\hat{\varepsilon}^{q}} \circ u_{\hat{\varepsilon}}$ in the two terms $R_{\text {porMed }}^{(5)}$ and $R_{\text {corr }}^{(5)}$. This can be done by an estimation similar to the proof of Lemma 11 , this time using in particular (7), (10) and $\mathbf{E} C_{\alpha}^{2}<\infty$, which yields the bound

$$
\begin{aligned}
& \mathbf{E} R_{\text {porMed }}^{(5)}+\mathbf{E} R_{\text {corr }}^{(5)} \\
& \leq-\mathbf{E} \int_{0}^{T^{*}} \varphi(t) \int_{\mathbf{R}^{d}}\left|\left(\zeta_{\kappa}^{\varepsilon^{q}} \circ u_{\varepsilon}\right)^{m}(x, t)-\left(\zeta_{\kappa}^{\hat{\varepsilon}^{q}} \circ u_{\hat{\varepsilon}}\right)^{m}(x, t)\right| \Delta \bar{\xi}_{l}(x) \mathrm{d} x \mathrm{~d} t \\
& \quad-\mathbf{E} \int_{0}^{T^{*}} \varphi(t) \int_{\mathbf{R}^{d}} \frac{1}{2} v^{2}\left|\left(\zeta_{\kappa}^{\varepsilon^{q}} \circ u_{\varepsilon}\right)(x, t)-\left(\zeta_{\kappa}^{\hat{\varepsilon}^{q}} \circ u_{\hat{\varepsilon}}\right)(x, t)\right| \Delta \bar{\xi}_{l}(x) \mathrm{d} x \mathrm{~d} t \\
& \quad+C(\kappa) l^{-2}(\varepsilon \vee \hat{\varepsilon})^{\alpha} .
\end{aligned}
$$

However, it follows from $\varepsilon, \hat{\varepsilon} \leq \frac{\kappa}{2}$ that $\left(\zeta_{\kappa}^{\varepsilon^{q}} \circ u_{\varepsilon}\right)^{m}-\left(\zeta_{\kappa}^{\hat{\varepsilon}^{q}} \circ u_{\hat{\varepsilon}}\right)^{m} \in H_{0}^{1}(D)$ as well as $\left(\zeta_{\kappa}^{\varepsilon^{q}} \circ u_{\varepsilon}\right)-\left(\zeta_{\kappa}^{\hat{\varepsilon}^{q}} \circ u_{\hat{\varepsilon}}\right) \in H_{0}^{1}(D)$. An integration by parts together with condition $\left.i i\right)$ of the spatial cut-off function $\bar{\xi}$ then entails

$$
\begin{aligned}
\mathbf{E} R_{\text {porMed }}^{(5)}+\mathbf{E} R_{\text {corr }}^{(5)} & \\
\leq & -\mathbf{E} \int_{0}^{T^{*}} \varphi(t) \int_{\mathbf{R}^{d}} \nabla\left|\left(\zeta_{\kappa}^{\varepsilon^{q}} \circ u_{\varepsilon}\right)^{m}(x, t)-\left(\zeta_{\kappa}^{\hat{\varepsilon}^{q}} \circ u_{\hat{\varepsilon}}\right)^{m}(x, t)\right| \cdot \nabla \bar{\xi}_{l}(x) \mathrm{d} x \mathrm{~d} t \\
& -\mathbf{E} \int_{0}^{T^{*}} \varphi(t) \int_{\mathbf{R}^{d}} \frac{1}{2} v^{2} \nabla\left|\left(\zeta_{\kappa}^{\varepsilon^{q}} \circ u_{\varepsilon}\right)(x, t)-\left(\zeta_{\kappa}^{\hat{\varepsilon}^{q}} \circ u_{\hat{\varepsilon}}\right)(x, t)\right| \cdot \nabla \bar{\xi}_{l}(x) \mathrm{d} x \mathrm{~d} t \\
& +C(\kappa) l^{-2}(\varepsilon \vee \hat{\varepsilon})^{\alpha} \\
\leq & -\mathbf{E} \int_{0}^{T^{*}} \varphi(t) \int_{\mathbf{R}^{d}} \nabla\left|\left(\zeta_{\kappa}^{\varepsilon^{q}} \circ u_{\varepsilon}\right)^{m}(x, t)-\left(\zeta_{\kappa}^{\hat{\varepsilon}^{q}} \circ u_{\hat{\varepsilon}}\right)^{m}(x, t)\right| \cdot \nabla\left(\bar{\xi}_{l}-\bar{\xi}\right)(x) \mathrm{d} x \mathrm{~d} t \\
& -\mathbf{E} \int_{0}^{T^{*}} \varphi(t) \int_{\mathbf{R}^{d}} \frac{1}{2} v^{2} \nabla\left|\left(\zeta_{\kappa}^{\varepsilon^{q}} \circ u_{\varepsilon}\right)(x, t)-\left(\zeta_{\kappa}^{\hat{\varepsilon}^{q}} \circ u_{\hat{\varepsilon}}\right)(x, t)\right| \cdot \nabla\left(\bar{\xi}_{l}-\bar{\xi}\right)(x) \mathrm{d} x \mathrm{~d} t \\
& +C(\kappa) l^{-2}(\varepsilon \vee \hat{\varepsilon})^{\alpha} .
\end{aligned}
$$

It remains to bound the two terms featuring the difference $\nabla \bar{\xi}_{l}-\nabla \bar{\xi}$. By continuity of translations in $L^{2}$, however, together with the a priori estimate (16) we find some 
small enough $l(\bar{\xi})>0$ such that

$$
l<l(\bar{\xi}) \Rightarrow\left\|\bar{\xi}_{l}-\bar{\xi}\right\|_{H^{1}\left(\mathbf{R}^{d}\right)} \leq \kappa^{\frac{m+1}{2}},
$$

holds true, and therefore in particular the desired estimate (79) for some absolute constant $\bar{C}>0$.

We continue with the proof of Proposition 5. It is straightforward to estimate

$$
\left|-\mathbf{E} R_{\mathrm{dt}}^{(3)}-\mathbf{E} \int_{0}^{T^{*}} \frac{\mathrm{d}}{\mathrm{d} t} \varphi(t) \int_{\mathbf{R}^{d}} \int_{\mathbf{R}^{d}}\right| v_{\varepsilon}(x, t)-v_{\hat{\varepsilon}}(x, t)\left|\gamma_{\theta}(x-y) \bar{\xi}(x) \mathrm{d} y \mathrm{~d} x \mathrm{~d} t\right| \leq \bar{C} \kappa
$$

for all $l$ subject to $(80)$ and all $\varphi \in C_{\mathrm{cpt}}^{\infty}\left(\left(0, T^{*}\right) ;[0,1]\right)$ with $\|\varphi\|_{W^{1,1}\left(0, T^{*}\right)} \leq \bar{C}$. In summary, we thus obtain together with (79) the estimate

$$
\begin{aligned}
-\mathbf{E} R_{\mathrm{dt}}^{(4)} & :=-\mathbf{E} \int_{0}^{T^{*}} \frac{\mathrm{d}}{\mathrm{d} t} \varphi(t) \int_{\mathbf{R}^{d}} \int_{\mathbf{R}^{d}}\left|v_{\varepsilon}(x, t)-v_{\hat{\varepsilon}}(y, t)\right| \gamma_{\theta}(x-y) \bar{\xi}(x) \mathrm{d} y \mathrm{~d} x \mathrm{~d} t \\
& \leq C(\kappa) l^{-1} \theta^{-1}(\varepsilon \vee \hat{\varepsilon})^{q}+C(\kappa) l^{-2}(\varepsilon \vee \hat{\varepsilon})^{\alpha}+C(\kappa) l^{-3} \theta+\bar{C} \kappa
\end{aligned}
$$

for all $\varphi \in C_{\mathrm{cpt}}^{\infty}\left(\left(0, T^{*}\right) ;[0,1]\right)$ with $\|\varphi\|_{W^{1,1}\left(0, T^{*}\right)} \leq \bar{C}$, all $(\theta, l)$ subject to (52) resp. (80), and all $\varepsilon, \hat{\varepsilon} \leq \frac{\kappa}{2}$.

In the next step we take care of the term $\mathbf{E} R_{\mathrm{dt}}^{(4)}$. Employing the same argument leading to [7, (4.20)] (instead of using [7, Lemma 3.2] to treat the initial condition we can also rely on the continuity down to $t=0$ thanks to Lemma 4 ) we infer that the estimate (81) entails the bound (recall that the initial condition is deterministic)

$$
\begin{aligned}
& \mathbf{E} \int_{D} \int_{D}\left|v_{\varepsilon}(x, T)-v_{\hat{\varepsilon}}(y, T)\right| \gamma_{\theta}(x-y) \bar{\xi}(x) \mathrm{d} y \mathrm{~d} x \\
& \leq \int_{D} \int_{D}\left|v_{\varepsilon}(x, 0)-v_{\hat{\varepsilon}}(y, 0)\right| \gamma_{\theta}(x-y) \bar{\xi}(x) \mathrm{d} y \mathrm{~d} x \\
& \quad+C(\kappa) l^{-1} \theta^{-1}(\varepsilon \vee \hat{\varepsilon})^{q}+C(\kappa) l^{-2}(\varepsilon \vee \hat{\varepsilon})^{\alpha}+C(\kappa) l^{-3} \theta+\bar{C} \kappa
\end{aligned}
$$

for all $T \in\left[0, T^{*}\right]$, all $(\theta, l)$ subject to (52) resp. (80), and all $\varepsilon, \hat{\varepsilon} \leq \frac{\kappa}{2}$. We then estimate for all $T \in\left[\kappa, T^{*}\right]$ by means of (17), (19) and $\|\bar{\xi}\|_{L^{\infty}(D)} \leq 1$

$$
\begin{aligned}
& \left|\mathbf{E} \int_{D} \int_{D}\right| v_{\varepsilon}(x, T)-v_{\hat{\varepsilon}}(y, T)\left|\gamma_{\theta}(x-y) \bar{\xi}(x) \mathrm{d} y \mathrm{~d} x-\mathbf{E} \int_{D}\right| v_{\varepsilon}(x, T)-v_{\hat{\varepsilon}}(x, T)|\bar{\xi}(x) \mathrm{d} x| \\
& \quad \leq \mathbf{E} \int_{D} \int_{D}\left|v_{\hat{\varepsilon}}(y+x, T)-v_{\hat{\varepsilon}}(x, T)\right| \gamma_{\theta}(y) \bar{\xi}(x) \mathrm{d} x \mathrm{~d} y \\
& \quad \leq C \theta \mathbf{E} \int_{0}^{1} \int_{\mathbf{R}^{d}} \int_{\mathbf{R}^{d}}\left|\nabla u_{\hat{\varepsilon}}^{\leftarrow}(x-r y, T)\right| \gamma_{\theta}(y) \mathrm{d} x \mathrm{~d} y \mathrm{~d} r \\
& \quad \leq C(\kappa) \theta \mathbf{E} \int_{D} u_{\hat{\varepsilon}}^{m-1}\left|\nabla u_{\hat{\varepsilon}}\right| \mathrm{d} x \leq C(\kappa) \hat{\varepsilon}^{-\beta} \theta .
\end{aligned}
$$


Since we may assume in this argument without loss of generality that $\varepsilon \leq \hat{\varepsilon}$ (otherwise, switch the roles of $v_{\hat{\varepsilon}}$ and $v_{\varepsilon}$ in the previous estimate) we obtain together with an analogous estimate based on the regularity of the initial condition and the fact that $\bar{\xi} \equiv 1$ on $K$ as well as $\bar{\xi} \geq 0$ on $D$,

$$
\begin{aligned}
& \mathbf{E} \int_{K}\left|v_{\varepsilon}(x, T)-v_{\hat{\varepsilon}}(x, T)\right| \mathrm{d} x \\
& \quad \leq \int_{D}\left|v_{\varepsilon}(x, 0)-v_{\hat{\varepsilon}}(x, 0)\right| \mathrm{d} x+C(\kappa) l^{-1} \theta^{-1}(\varepsilon \vee \hat{\varepsilon})^{q}+C(\kappa) l^{-2}(\varepsilon \vee \hat{\varepsilon})^{\alpha} \\
& \quad+C(\kappa)(\varepsilon \vee \hat{\varepsilon})^{-\beta} \theta+C(\kappa) l^{-3} \theta+\bar{C} \kappa
\end{aligned}
$$

for all $T \in\left[\kappa, T^{*}\right]$, all $(\theta, l)$ subject to (52) resp. (80), and all $\varepsilon, \hat{\varepsilon} \leq \frac{\kappa}{2}$. As a consequence of (21) and the triangle inequality, we may finally switch from $v_{\varepsilon}(\cdot)=$ $\zeta_{\kappa}^{\varepsilon^{q}}\left(u_{\varepsilon}^{\leftarrow}(\cdot)\right)=\kappa+\zeta_{\varepsilon^{q}}\left(u_{\varepsilon}^{\leftarrow}(\cdot)-\kappa\right)$ to $\kappa \vee u_{\varepsilon}^{\leftarrow}(\cdot)=\kappa+\left(u_{\varepsilon}^{\leftarrow}(\cdot)-\kappa\right)_{+}$, which yields

$$
\begin{aligned}
\mathbf{E} & \int_{K}\left|\kappa \vee u_{\varepsilon}^{\leftarrow}(x, T)-\kappa \vee u_{\hat{\varepsilon}}^{\leftarrow}(x, T)\right| \mathrm{d} x \\
\leq & \mathbf{E} \int_{K}\left|v_{\varepsilon}(x, T)-v_{\hat{\varepsilon}}(x, T)\right| \mathrm{d} x+C \mathcal{L}^{d}(D)(\varepsilon \vee \hat{\varepsilon})^{q} \\
\leq & \int_{D}\left|\kappa \vee u_{\varepsilon}^{\leftarrow}(x, 0)-\kappa \vee u_{\hat{\varepsilon}}^{\leftarrow}(x, 0)\right| \mathrm{d} x+C(\kappa) l^{-1} \theta^{-1}(\varepsilon \vee \hat{\varepsilon})^{q} \\
& +C(\kappa) l^{-2}(\varepsilon \vee \hat{\varepsilon})^{\alpha}+C(\kappa)(\varepsilon \vee \hat{\varepsilon})^{-\beta} \theta+C(\kappa) l^{-3} \theta+\bar{C} \kappa
\end{aligned}
$$

for all $T \in\left[\kappa, T^{*}\right]$, all $(\theta, l)$ subject to (52) resp. (80), and all $\varepsilon, \hat{\varepsilon} \leq \frac{\kappa}{2}$. We eventually arrived at the last step of the proof.

In light of the right hand side terms in (84) we first define $\theta:=(\varepsilon \vee \hat{\varepsilon})^{\beta+1}$, then fix $\bar{\vartheta}>0$ and $q>0$ such that $2 \bar{\vartheta}<\alpha, 3 \bar{\vartheta}<\beta+1$ as well as $\beta+1+\bar{\vartheta}<q$, and finally define $l:=(\varepsilon \vee \hat{\varepsilon})^{\bar{\vartheta}}$. Choosing $\varepsilon_{0}>0$ small enough, we can ensure that $(\theta, l)$ satisfy (52) resp. (80). Hence, these choices guarantee that we may infer from (84) a bound of the type (23) with right hand side terms $C(\kappa)(\varepsilon \vee \hat{\varepsilon})^{2 \vartheta}$ for some suitable exponent $\vartheta>0$. Choosing $\varepsilon_{0}$ even smaller, if needed, we can avoid the dependence of the constant on the data by sacrificing a power $\vartheta$, which entails (23). This concludes the proof of Proposition 5.

\subsection{Proof of Corollary 6 ( $L^{1}$ convergence of shifted densities)}

Let $\delta>0$ be fixed but arbitrary. By the triangle inequality we may estimate

$$
\begin{aligned}
& \sup _{T \in\left[\kappa, T^{*}\right]} \mathbf{E} \int_{D}\left|u_{\varepsilon}^{\leftarrow}(T)-u_{\hat{\varepsilon}}^{\leftarrow}(T)\right| \mathrm{d} x \\
& \leq \sup _{T \in\left[\kappa, T^{*}\right]} \mathbf{E} \int_{D}\left|u_{\varepsilon}^{\leftarrow}(T)\right| \chi_{\left\{u_{\varepsilon}(T)<\kappa\right\}}+\left|u_{\hat{\varepsilon}}^{\leftarrow}(T)\right| \chi_{\left\{u_{\hat{\varepsilon}}(T)<\kappa\right\}} \mathrm{d} x
\end{aligned}
$$




$$
+\sup _{T \in\left[\kappa, T^{*}\right]} \mathbf{E} \int_{D}\left|\kappa \vee u_{\varepsilon}^{\leftarrow}(x, T)-\kappa \vee u_{\hat{\varepsilon}}^{\leftarrow}(x, T)\right| \mathrm{d} x
$$

for all $\kappa \in\left(0, T^{*} \wedge 1\right)$ and all $\varepsilon, \hat{\varepsilon} \leq \frac{\kappa}{2}$. Hence, it follows from splitting $D=$ $K \cup(D \backslash K)$ together with the bounds (15) and (23) as well as the definition (22) of the shifted densities that

$$
\begin{aligned}
& \sup _{T \in\left[\kappa, T^{*}\right]} \mathbf{E} \int_{D}\left|u_{\varepsilon}^{\leftarrow}(T)-u_{\hat{\varepsilon}}^{\leftarrow}(T)\right| \mathrm{d} x \\
& \leq 2 \mathcal{L}^{d}(D) \kappa+2 \mathcal{L}^{d}(D \backslash K)\left(\kappa \vee\left(1+\left\|u_{0}\right\|_{L^{\infty}(D)}\right)\right)+\bar{C}(\varepsilon \vee \hat{\varepsilon})^{\vartheta}+\bar{C} \kappa \\
& \quad+\mathbf{E} \int_{D}\left|\kappa \vee\left(u_{0}(x)+\varepsilon\right)-\kappa \vee\left(u_{0}(x)+\hat{\varepsilon}\right)\right| \mathrm{d} x .
\end{aligned}
$$

The term with the initial data is estimated similarly by

$$
\mathbf{E} \int_{D}\left|\kappa \vee\left(u_{0}(x)+\varepsilon\right)-\kappa \vee\left(u_{0}(x)+\hat{\varepsilon}\right)\right| \mathrm{d} x \leq 2 \mathcal{L}^{d}(D) \kappa+\mathcal{L}^{d}(D)|\varepsilon-\hat{\varepsilon}|
$$

for all $\kappa>0$ and all $\varepsilon, \hat{\varepsilon} \leq \frac{\kappa}{2}$. Choosing first $\kappa<\tau$ sufficiently small such that $\left(4 \mathcal{L}^{d}(D)+\bar{C}\right) \kappa \leq \frac{\delta}{2}$, we may then fix a large enough compact set $K \subset D$ and some small enough $\varepsilon_{0}(\kappa, K)$ such that the bound

$$
\sup _{T \in\left[\tau, T^{*}\right]} \mathbf{E} \int_{D}\left|u_{\varepsilon}^{\leftarrow}(T)-u_{\hat{\varepsilon}}^{\leftarrow}(T)\right| \mathrm{d} x \leq \delta
$$

holds true for all $\varepsilon, \hat{\varepsilon} \leq \varepsilon_{0}$. This proves that the sequence of shifted densities $u_{\varepsilon}^{\leftarrow}$ is a Cauchy sequence in the space $C\left(\left[\tau, T^{*}\right] ; L^{1}\left(\Omega \times D, \mathbf{P} \otimes \mathcal{L}^{d}\right)\right)$ for all $\tau>0$. The corresponding assertion in the space $L^{1}\left(\left[0, T^{*}\right] ; L^{1}\left(\Omega \times D, \mathbf{P} \otimes \mathcal{L}^{d}\right)\right)$ is proved similarly based on the additional estimate

$$
\begin{aligned}
& \mathbf{E} \int_{0}^{T^{*}} \int_{D}\left|u_{\varepsilon}^{\leftarrow}-u_{\hat{\varepsilon}}^{\leftarrow}\right| \mathrm{d} x \mathrm{~d} t \\
& \quad \leq 2 \mathcal{L}^{d}(D) \kappa\left(1+\left\|u_{0}\right\|_{L^{\infty}(D)}\right)+\left(T^{*}-\kappa\right) \sup _{T \in\left[\kappa, T^{*}\right]} \mathbf{E} \int_{D}\left|u_{\varepsilon}^{\leftarrow}(T)-u_{\hat{\varepsilon}}^{\leftarrow}(T)\right| \mathrm{d} x
\end{aligned}
$$

Let us denote the corresponding limit in $L^{1}\left(\left[0, T^{*}\right] ; L^{1}\left(\Omega \times D, \mathbf{P} \otimes \mathcal{L}^{d}\right)\right)$ by $u$.

On the other side, it follows immediately from the bound (15) that the sequence of densities $u_{\varepsilon}$ has a weak limit in the space $L^{m+1}\left(\Omega_{T^{*}}, \mathcal{P}_{T^{*}} ; L^{m+1}(D)\right)$, which we denote by $\bar{u}$. It remains to verify that $u=\bar{u}$. To this end, let $\phi \in C_{\mathrm{cpt}}^{\infty}\left(D \times\left[0, T^{*}\right]\right)$ and $A \in \mathcal{F}_{T^{*}}$ be fixed. We then have

$$
\mathbf{E} \chi_{A} \int_{0}^{T^{*}} \int_{D}(u-\bar{u}) \phi \mathrm{d} x \mathrm{~d} t=\lim _{\varepsilon \rightarrow 0} \mathbf{E} \chi_{A} \int_{0}^{T^{*}} \int_{D}\left(u_{\varepsilon}^{\leftarrow}-u_{\varepsilon}\right) \phi \mathrm{d} x \mathrm{~d} t
$$


By a simple change of variables and the definition (22) we may write

$$
\begin{aligned}
& \mathbf{E} \int_{0}^{T^{*}} \int_{D}\left(u_{\varepsilon}^{\leftarrow}-u_{\varepsilon}\right) \phi \mathrm{d} x \mathrm{~d} t \\
& \quad=\mathbf{E} \int_{0}^{T^{*}} \int_{\mathbf{R}^{d}} u_{\varepsilon}(x, t)\left(\phi\left(x-v\left(B_{t}-B_{t}^{\varepsilon}\right), t\right)-\phi(x, t)\right) \mathrm{d} x \mathrm{~d} t .
\end{aligned}
$$

Exploiting (7), (10) and (15) we further estimate

$$
\begin{aligned}
& \left|\mathbf{E} \int_{0}^{T^{*}} \int_{\mathbf{R}^{d}} u_{\varepsilon}(x, t)\left(\phi\left(x-v\left(B_{t}-B_{t}^{\varepsilon}\right), t\right)-\phi(x, t)\right) \mathrm{d} x \mathrm{~d} t\right| \\
& \quad \leq C\|\nabla \phi\|_{L^{\infty}\left(D \times\left[0, T^{*}\right]\right)} \varepsilon^{\alpha} \mathbf{E} C_{\alpha} \leq C \varepsilon^{\alpha} .
\end{aligned}
$$

Hence, we may infer that

$$
\mathbf{E} \chi_{A} \int_{0}^{T^{*}} \int_{D}(u-\bar{u}) \phi \mathrm{d} x \mathrm{~d} t=0
$$

holds true for all $\phi \in C_{\mathrm{cpt}}^{\infty}\left(D \times\left[0, T^{*}\right]\right)$ and $A \in \mathcal{F}_{T^{*}}$. This shows that $u=\bar{u}$ and thus concludes the proof of Corollary 6 .

4.4 Proof of Proposition 7 (Recovering the unique weak solution)

Let a test function $\phi \in C_{\mathrm{cpt}}^{\infty}(D)$ be fixed, and define $K:=\operatorname{supp} \phi$. Fix also an integer $M \geq 1$, and let $\mathcal{C}_{\alpha}$ be the square integrable random variable of (10). Let $\delta \in(0,1)$ be such that $\left\{x \in \mathbf{R}^{d}: \operatorname{dist}(x, K) \leq \delta\right\} \subset D$. Let $\varepsilon^{\prime}=\varepsilon^{\prime}(M, \delta)$ be the constant from (11). By Itô's formula, the definition (22) and the fact that the $u_{\varepsilon}$ solve (12) classically we have for all $\varepsilon \leq \varepsilon^{\prime}$ and all measurable $A \in \mathcal{F}_{T^{*}}$ (cf. the argument in the first step of the proof of Proposition 5)

$$
\begin{aligned}
\mathbf{E} \chi_{\left\{\mathcal{C}_{\alpha} \leq M\right\}} \chi_{A} \int_{D} u_{\varepsilon}^{\leftarrow}(x, T) \phi(x) \mathrm{d} x-\mathbf{E} \chi_{\left\{\mathcal{C}_{\alpha} \leq M\right\}} \chi_{A} \int_{D}\left(u_{0}(x)+\varepsilon\right) \phi(x) \mathrm{d} x \\
=\mathbf{E} \chi_{\left\{\mathcal{C}_{\alpha} \leq M\right\}} \chi_{A} \int_{0}^{T} \int_{D}\left(\Delta\left(u_{\varepsilon}^{\leftarrow}\right)^{m}(x, t)+\frac{1}{2} v^{2} \Delta u_{\varepsilon}^{\leftarrow}(x, t)\right) \phi(x) \mathrm{d} x \mathrm{~d} t \\
\quad+\mathbf{E} \chi_{\left\{\mathcal{C}_{\alpha} \leq M\right\}} \chi_{A} \int_{0}^{T} \int_{D} v \nabla u_{\varepsilon}^{\leftarrow}(x, t) \phi(x) \mathrm{d} x \mathrm{~d} B_{t}
\end{aligned}
$$

for all $T \in\left(0, T^{*}\right)$. Define the shifted test function

$$
\phi_{\varepsilon}^{\leftarrow}(x, t):=\phi\left(x-v\left(B_{t}-B_{t}^{\varepsilon}\right)\right)
$$


so that we obtain by a simple change of variables

$$
\begin{aligned}
\mathbf{E} \chi_{\left\{\mathcal{C}_{\alpha} \leq M\right\}} \chi_{A} \int_{D} u_{\varepsilon}^{\leftarrow}(x, T) \phi(x) \mathrm{d} x-\mathbf{E} \chi_{\left\{\mathcal{C}_{\alpha} \leq M\right\}} \chi_{A} \int_{D}\left(u_{0}(x)+\varepsilon\right) \phi(x) \mathrm{d} x \\
=\mathbf{E} \chi_{\left\{\mathcal{C}_{\alpha} \leq M\right\}} \chi_{A} \int_{0}^{T} \int_{D}\left(\Delta u_{\varepsilon}^{m}(x, t)+\frac{1}{2} v^{2} \Delta u_{\varepsilon}(x, t)\right) \phi_{\varepsilon}^{\leftarrow}(x, t) \mathrm{d} x \mathrm{~d} t \\
+\mathbf{E} \chi_{\left\{\mathcal{C}_{\alpha} \leq M\right\}} \chi_{A} \int_{0}^{T} \int_{D} v \nabla u_{\varepsilon}(x, t) \phi_{\varepsilon}^{\leftarrow}(x, t) \mathrm{d} x \mathrm{~d} B_{t} .
\end{aligned}
$$

Note that as a consequence of (11) we have almost surely on $\left\{\mathcal{C}_{\alpha} \leq M\right\}$ for all $\varepsilon \leq \varepsilon^{\prime}$ and all $t \in\left[0, T^{*}\right]$ that $\operatorname{supp} \phi_{\varepsilon}^{\leftarrow}(\cdot, t) \subset \subset D$. Hence, integrating by parts on the right hand side in (86) does not produce any boundary integrals so that after reversing the change of variables we obtain the identity

$$
\begin{aligned}
\mathbf{E} \chi_{\left\{\mathcal{C}_{\alpha} \leq M\right\}} \chi_{A} \int_{D} u_{\varepsilon}^{\leftarrow}(x, T) \phi(x) \mathrm{d} x-\mathbf{E} \chi_{\left\{\mathcal{C}_{\alpha} \leq M\right\}} \chi_{A} \int_{D}\left(u_{0}(x)+\varepsilon\right) \phi(x) \mathrm{d} x \\
=\mathbf{E} \chi_{\left\{\mathcal{C}_{\alpha} \leq M\right\}} \chi_{A} \int_{0}^{T} \int_{D}\left(\left(u_{\varepsilon}^{\leftarrow}\right)^{m}(x, t)+\frac{1}{2} v^{2} u_{\varepsilon}^{\leftarrow}(x, t)\right) \Delta \phi(x) \mathrm{d} x \mathrm{~d} t \\
-\mathbf{E} \chi_{\left\{\mathcal{C}_{\alpha} \leq M\right\}} \chi_{A} \int_{0}^{T} \int_{D} v u_{\varepsilon}^{\leftarrow}(x, t) \nabla \phi(x) \mathrm{d} x \mathrm{~d} B_{t} .
\end{aligned}
$$

We aim to pass to the limit $\varepsilon_{0} \geq \varepsilon \rightarrow 0$ in all four terms. This is possible by the convergence of the shifted densities $u_{\varepsilon}^{\leftarrow}$ in $C\left(\left[\tau, T^{*}\right] ; L^{1}\left(\Omega \times D, \mathbf{P} \otimes \mathcal{L}^{d}\right)\right)$ as well as in $L^{1}\left(\left[0, T^{*}\right] ; L^{1}\left(\Omega \times D, \mathbf{P} \otimes \mathcal{L}^{d}\right)\right)$, see Corollary 6 . Because of the uniform bound (15) the convergence in $L^{1}$ can actually be lifted to convergence in any $L^{q}\left(\left[0, T^{*}\right] ; L^{q}\left(\Omega \times D, \mathbf{P} \otimes \mathcal{L}^{d}\right)\right), q \in(1, \infty)$, which makes the limit passage possible in the non-linear diffusion term as well as the noise term (using for the latter, e.g., the Burkholder-Davis-Gundy inequality). In summary, we obtain from letting $\varepsilon_{0} \geq \varepsilon \rightarrow 0$ the identity

$$
\begin{gathered}
\mathbf{E} \chi_{\left\{\mathcal{C}_{\alpha} \leq M\right\}} \chi_{A} \int_{D} u(x, T) \phi(x) \mathrm{d} x-\mathbf{E} \chi_{\left\{\mathcal{C}_{\alpha} \leq M\right\}} \chi_{A} \int_{D} u_{0}(x) \phi(x) \mathrm{d} x \\
=\mathbf{E} \chi_{\left\{\mathcal{C}_{\alpha} \leq M\right\}} \chi_{A} \int_{0}^{T} \int_{D}\left(u^{m}(x, t)+\frac{1}{2} v^{2} u(x, t)\right) \Delta \phi(x) \mathrm{d} x \mathrm{~d} t \\
-\mathbf{E} \chi_{\left\{\mathcal{C}_{\alpha} \leq M\right\}} \chi_{A} \int_{0}^{T} \int_{D} v u(x, t) \nabla \phi(x) \mathrm{d} x \mathrm{~d} B_{t}
\end{gathered}
$$

for all $T \in\left(0, T^{*}\right)$. Since $M \geq 1$ as well as $A \in \mathcal{F}_{T^{*}}$ were arbitrary, and the random variable $\mathcal{C}_{\alpha}$ is integrable, we thus recover (4). This concludes the proof of Proposition 7 since the asserted bounds for $u$ follow immediately from (15). 


\section{Finite time extinction property}

\subsection{Viscosity theory: maximal subsolution in a rough domain}

From now on we will restrict ourselves to the one-dimensional setting $d=1$. To start we recall some language from viscosity theory (cf. [6]). In particular, we aim to make precise what we mean by a subsolution to the Cauchy-Dirichlet problem (24)-(26) of the porous medium equation with two copies of a Brownian path as lateral boundary; and analogously for the corresponding regularized problem (27)-(29).

In a first step, we introduce the relevant notions for the full space problem. The porous medium operator in terms of the pressure variable is encoded by the functional

$$
F:[0, \infty) \times \mathbf{R} \times \mathbf{R} \rightarrow \mathbf{R}, \quad(r, q, X) \mapsto(m-1) r X+|q|^{2} .
$$

Let $T^{*} \in(0, \infty]$ be a time horizon and $p: \mathbf{R} \times\left(0, T^{*}\right) \rightarrow \mathbf{R}$ be a function. The parabolic semijet $\mathcal{J}^{2,+} p(x, t)$ of $p$ at $(x, t) \in \mathbf{R} \times\left(0, T^{*}\right)$ is the set of all $(q, a, X) \in$ $\mathbf{R}^{3}$ such that

$$
\begin{aligned}
p(y, s) \leq & p(x, t)+a(s-t)+q(y-x)+\frac{1}{2} X(y-x)^{2} \\
& +o\left(|s-t|+|y-x|^{2}\right) \text { as } \mathbf{R} \times(0, t] \ni(y, s) \rightarrow(x, t) .
\end{aligned}
$$

Note that the condition in (89) is a slightly weaker test than the one of $[6,(8.1)]$ since we are only allowing for $s \leq t$. This minor technicality turns out to be convenient proving that subsolutions to, say, (24)-(26) in the sense of Definition 14 below are also subsolutions to (90) and (91). Moreover, (89) is in accordance with the definition of subsolutions in [5, Definition 1].

Definition 13 Let $T^{*} \in(0, \infty]$ be a time horizon and $p_{0} \in C_{\mathrm{cpt}}^{\infty}(\mathbf{R} ;[0, \infty))$ be an initial pressure. An upper-semicontinuous function $p: \mathbf{R} \times\left[0, T^{*}\right) \rightarrow[0, \infty)$ is called $a$ subsolution for the Cauchy problem of the porous medium equation with initial pressure $p_{0}$

$$
\begin{aligned}
\partial_{t} p & =(m-1) p \partial_{x x} p+\left|\partial_{x} p\right|^{2}, & (x, t) & \in \mathbf{R} \times\left(0, T^{*}\right), \\
p(x, 0) & =p_{0}(x), & x & \in \mathbf{R},
\end{aligned}
$$

if it holds

$$
a-F(p(x, t), q, X) \leq 0
$$

for all $(x, t) \in \mathbf{R} \times\left(0, T^{*}\right)$ and all $(q, a, X) \in \mathcal{J}^{2,+} p(x, t)$, as well as if it holds $p(x, 0) \leq p_{0}(x)$ for all $x \in \mathbf{R}$.

In the language of [6], the functional $a-F(r, p, X)$ encoding the porous medium equation in terms of the pressure variable is not proper. But note that it is at least degenerate elliptic. Since we will only deal with subsolutions (or for the regularized 
problem with classical solutions), this is of no concern for us (cf. [5] or [20] for a viscosity theory of the Cauchy problem of the deterministic porous medium equation).

We continue with the notion of subsolutions to (24)-(26) (resp. (27)-(29)). Since we have to incorporate Brownian paths as the lateral boundary, the following constructions are of course random. However, all results of this section turn out to be purely deterministic consequences of the probabilistic facts (8), (9), (10) and (11). In other words, we proceed with constructions to be understood in a pathwise sense.

The functional for the lateral boundary condition of the limit problem is simply defined by $C: \mathbf{R} \rightarrow \mathbf{R}, r \mapsto r$. The one for the regularized problem is given by $C_{\varepsilon}: \mathbf{R} \rightarrow \mathbf{R}, r \mapsto r-\frac{m}{m-1} \varepsilon^{m-1}$. We then introduce a lower-semicontinuous functional

$$
\begin{aligned}
& G^{-}: \mathbf{R} \times\left(0, T^{*}\right) \times[0, \infty) \times \mathbf{R}^{3} \rightarrow \mathbf{R} \\
& (x, t, r, q, a, X) \mapsto \begin{cases}a-F(r, q, X), & (x, t) \in \bigcup_{t \in\left(0, T^{*}\right)}\left(v B_{t}+I\right) \times\{t\}, \\
(a-F(r, q, X)) \wedge C(r), & (x, t) \in \bigcup_{t \in\left(0, T^{*}\right)}\left(v B_{t}+\partial I\right) \times\{t\}, \\
C(r), & \text { else, }\end{cases}
\end{aligned}
$$

encoding the equations (24) and (26). The corresponding functional for the regularized problem, i.e., encoding (27) and (29), is given by

$$
\begin{aligned}
G_{\varepsilon}^{-} & : \mathbf{R} \times\left(0, T^{*}\right) \times[0, \infty) \times \mathbf{R}^{3} \rightarrow \mathbf{R} \\
& (x, t, r, q, a, X) \mapsto \begin{cases}a-F(r, q, X), & (x, t) \in \bigcup_{t \in\left(0, T^{*}\right)}\left(v B_{t}^{\varepsilon}+I\right) \times\{t\}, \\
C_{\varepsilon}(r), & \text { else. }\end{cases}
\end{aligned}
$$

Definition 14 Let $T^{*} \in(0, \infty]$ be a time horizon, $I \subset \mathbf{R}$ be a bounded interval and $\bar{p}_{0} \in C_{\mathrm{cpt}}^{\infty}(I ;[0, \infty))$ be an initial pressure. An upper-semicontinuous function $\bar{p}: \mathbf{R} \times\left[0, T^{*}\right) \rightarrow[0, \infty)$ is called a subsolution for the Cauchy-Dirichlet problem (24)-(26) with initial pressure $\bar{p}_{0}$ if

$$
G^{-}(x, t, \bar{p}(x, t), q, a, X) \leq 0
$$

holds true for all $(x, t) \in \mathbf{R} \times\left(0, T^{*}\right)$ and all $(q, a, X) \in \mathcal{J}^{2,+} \bar{p}(x, t)$, as well as if $\bar{p}(x, 0) \leq \bar{p}_{0}(x)$ is satisfied for all $x \in \mathbf{R}$.

Analogously, we call an upper-semicontinuous function $\bar{p}_{\varepsilon}: \mathbf{R} \times\left[0, T^{*}\right) \rightarrow[0, \infty)$ a subsolution for the Cauchy-Dirichlet problem (27)-(29) if

$$
G_{\varepsilon}^{-}\left(x, t, \bar{p}_{\varepsilon}(x, t), q, a, X\right) \leq 0
$$

holds true for all $(x, t) \in \mathbf{R} \times\left(0, T^{*}\right)$ and all $(q, a, X) \in \mathcal{J}^{2,+} \bar{p}_{\varepsilon}(x, t)$, as well as if $\bar{p}_{\varepsilon}(x, 0) \leq \bar{p}_{0, \varepsilon}(x)$ is satisfied for all $x \in \mathbf{R}$. 
The reasons for relaxing (24)-(26) (resp. (27)-(29)) to the full space setting are twofold. On one hand, working in this framework turns out to be convenient when studying (any sort of) convergence of the subsolutions $\bar{p}_{\varepsilon}$ for the regularized problem. On the other hand, we also want to exploit the established results from the viscosity theory [5] (see also [20]) for the Dirichlet problem of the deterministic porous medium equation (90) and (91).

We proceed with a list of intermediate results needed to prove the main result of this work, Theorem 3 . The corresponding proofs will be provided afterwards. The first result concerns the construction of a subsolution to the regularized Cauchy-Dirichlet problem (27)-(29).

Lemma 15 Given $\varepsilon>0$, let $u_{\varepsilon}$ denote the unique weak solution to (12)-(14) with initial density $u_{0} \in C_{\mathrm{cpt}}^{\infty}(I ;[0, \infty))$ in the sense of Lemma 4. Define an associated pressure function as follows:

$$
\begin{aligned}
\bar{p}_{\varepsilon}: \mathbf{R} \times\left[0, T^{*}\right) \rightarrow[0, \infty) \\
\qquad(x, t) \mapsto \begin{cases}\frac{m}{m-1} u_{\varepsilon}\left(x-v B_{t}^{\varepsilon}, t\right)^{m-1}, & (x, t) \in \bigcup_{t \in\left[0, T^{*}\right)}\left(v B_{t}^{\varepsilon}+I\right) \times\{t\}, \\
\frac{m}{m-1} \varepsilon^{m-1}, & \text { else. }\end{cases}
\end{aligned}
$$

On a set with probability one the following then holds true:

For each $\varepsilon>0$, the associated pressure $\bar{p}_{\varepsilon}$ is continuous and a subsolution of the problem (27) and (29) with initial pressure $\bar{p}_{0, \varepsilon}(x):=\frac{m}{m-1}\left(u_{0}(x)+\varepsilon\right)^{m-1}$ in the sense of Definition 14. Moreover, we have the bounds

$$
\frac{m}{m-1} \varepsilon^{m-1} \leq \bar{p}_{\varepsilon}(x, t) \leq \frac{m}{m-1}\left(\varepsilon+\left\|u_{0}\right\|_{L^{\infty}(I)}\right)^{m-1}
$$

for all $(x, t) \in \mathbf{R} \times\left[0, T^{*}\right)$. Finally, $\bar{p}_{\varepsilon}$ is also a subsolution of the Cauchy problem (90)-(91) with initial pressure $p_{0, \varepsilon}(x):=\frac{m}{m-1}\left(u_{0}(x)+\varepsilon\right)^{m-1}$ in the sense of Definition 13.

In a next step, we construct on a set of probability one the maximal subsolution (also referred to as Perron's solution) $\bar{p}_{\max }$ of the limit Cauchy-Dirichlet problem (24)-(26) in the sense of Definition 14. Using standard arguments from viscosity theory, the main issue is to establish the existence of $a$ subsolution to (24)-(26). This will be done by means of the technique of semi-relaxed limits.

Proposition 16 For each $\varepsilon>0$ let $\bar{p}_{\varepsilon}$ denote the subsolution of (27)-(29) as constructed in Lemma 15. Define the upper semi-relaxed limit (with respect to backwards parabolic cylinders)

$$
\begin{aligned}
& \bar{p}_{\text {semi-rel }}(x, t) \\
& \quad:=\lim _{\varepsilon \rightarrow 0} \sup _{\hat{\varepsilon} \leq \varepsilon}\left\{\bar{p}_{\hat{\varepsilon}}(y, s):(y, s) \in \mathbf{R} \times\left[0, T^{*}\right),(y, s) \in(x-\varepsilon, x+\varepsilon) \times\left(t-\varepsilon^{2}, t\right]\right\}
\end{aligned}
$$

for all $(x, t) \in \mathbf{R} \times\left[0, T^{*}\right)$. Then the following holds true almost surely: 
The upper-semicontinuous envelope of $\bar{p}_{\text {semi-rel }}$ is a subsolution of (24)-(26) with initial pressure $\bar{p}_{0}(x):=\frac{m}{m-1} u_{0}(x)^{m-1}$ in the sense of Definition 14. Define

$$
\bar{p}_{\max }(x, t):=\sup \left\{\bar{p}(x, t): \bar{p} \text { is a subsol. of }(24)-(26),(x, t) \in \mathbf{R} \times\left[0, T^{*}\right)\right\} .
$$

Then $\bar{p}_{\max }(x, t)<\infty$ for all $(x, t) \in \mathbf{R} \times\left[0, T^{*}\right)$, and $\bar{p}_{\max }$ is a subsolution of (24)-(26) with initial pressure $\bar{p}_{0}(x):=\frac{m}{m-1} u_{0}(x)^{m-1}$ in the sense of Definition 14. Denoting by $p_{\mathrm{visc}} \in C\left(\mathbf{R} \times\left[0, T^{*}\right)\right)$ the viscosity solution of (90) and (91) with initial pressure $p_{0}(x):=\frac{m}{m-1} u_{0}(x)^{m-1}$ in the sense of $[5$, Definition 4$]$ we have

$$
\bar{p}_{\text {max }}(x, t) \leq p_{\text {visc }}(x, t)
$$

for all $(x, t) \in \mathbf{R} \times\left[0, T^{*}\right)$.

A crucial estimate for the proof of Theorem 3 is the content of the following result. The asserted bound is important in the sense that it serves to close the loop between the pathwise constructions performed in this section and the unique weak solution of the SPME (1)-(3).

Proposition 17 Let $u \in \mathbf{H}_{m+1}^{-1}(I)$ denote the unique weak solution of the CauchyDirichlet problem (1)-(3) with initial density $u_{0} \in C_{\mathrm{cpt}}^{\infty}(I ;[0, \infty))$ in the sense of Definition 1. Define an associated pressure function as follows:

$$
\begin{aligned}
p: \mathbf{R} \times\left(0, T^{*}\right) \rightarrow[0, \infty) & \\
(x, t) & \mapsto \begin{cases}\frac{m}{m-1} u\left(x-v B_{t}, t\right)^{m-1}, & (x, t) \in \bigcup_{t \in\left[0, T^{*}\right)}\left(v B_{t}+I\right) \times\{t\}, \\
0, & \text { else. }\end{cases}
\end{aligned}
$$

Let $\bar{p}_{\max }$ be the associated maximal subsolution of the problem (24)-(26) as constructed in Proposition 16. Then, for all $t \in\left(0, T^{*}\right)$ the bound

$$
p(\cdot, t) \leq \bar{p}_{\max }(\cdot, t)
$$

holds true almost surely almost everywhere in $\mathbf{R}$.

We have by now everything in place to proceed with the proofs.

5.2 Proof of Lemma 15 (Subsolution for the regularized problem)

The assertion that $\bar{p}_{\varepsilon} \in C\left(\mathbf{R} \times\left[0, T^{*}\right)\right)$ follows from the definition (97), the regularity of the Wong-Zakai approximation $u_{\varepsilon} \in C\left(\bar{I} \times\left[0, T^{*}\right)\right)$ and that $u_{\varepsilon}$ satisfies the lateral boundary condition (14) (cf. Lemma 4) pointwise. Moreover, $\bar{p}_{\varepsilon}(x, 0)=\bar{p}_{0, \varepsilon}(x)$ holds true for all $x \in \mathbf{R}$ because of (97), supp $u_{0} \subset I$ and $u_{\varepsilon}(x, 0)=u_{0}(x)+\varepsilon$. The upper and lower bound of (98) is a direct consequence of again the definition (97) and the upper and lower bound of (15). 
We next have to show that (96) is satisfied for all $(x, t) \in \mathbf{R} \times\left(0, T^{*}\right)$ and all $(q, a, X) \in \mathcal{J}^{2,+} \bar{p}_{\varepsilon}(x, t)$. The claim is trivial for $(x, t) \in \bigcup_{t \in\left(0, T^{*}\right)}\left(v B_{t}^{\varepsilon}+I\right) \times\{t\}$. Indeed, since $u_{\varepsilon}$ solves (12)-(14) classically in $I \times\left(0, T^{*}\right)$ and $B^{\varepsilon}$ is smooth we infer from (97), the chain rule and elementary computations that $\bar{p}_{\varepsilon}$ satisfies the porous medium equation $\partial_{t} \bar{p}_{\varepsilon}=\bar{p}_{\varepsilon} \partial_{x x} \bar{p}_{\varepsilon}+\left|\partial_{x} \bar{p}_{\varepsilon}\right|^{2}$ classically in the open space-time domain $\bigcup_{t \in\left(0, T^{*}\right)}\left(\nu B_{t}^{\varepsilon}+I\right) \times\{t\}$. If $(x, t) \in \bigcup_{t \in\left(0, T^{*}\right)}\left(\nu B_{t}^{\varepsilon}+(\mathbf{R} \backslash I)\right) \times\{t\}$, the claim is again trivial by the definition (97) of $\bar{p}_{\varepsilon}$ and (94). This proves (96).

We finally have to show that (92) holds true for all $(x, t) \in \mathbf{R} \times\left(0, T^{*}\right)$ and all $(q, a, X) \in \mathcal{J}^{2,+} \bar{p}_{\varepsilon}(x, t)$. By the previous reasoning, it just remains to consider the case of a space-time point on the lateral boundary $(x, t) \in \bigcup_{t \in\left(0, T^{*}\right)}\left(\nu B_{t}^{\varepsilon}+\partial I\right) \times\{t\}$. Without loss of generality we may assume that $(x, t)$ sits on the upper part of the lateral boundary. Let $(q, a, X) \in \mathcal{J}^{2,+} \bar{p}_{\varepsilon}(x, t)$ be fixed. Since $\bar{p}_{\varepsilon}$ satisfies the lateral boundary condition (29) pointwise, it follows from the definition of the parabolic semijet (89) that

$$
\bar{p}_{\varepsilon}(x-1 / k, t) \leq \frac{m}{m-1} \varepsilon^{m-1}-\frac{q}{k}+\frac{X}{2 k^{2}}+o\left(1 / k^{2}\right)
$$

as well as

$$
\frac{m}{m-1} \varepsilon^{m-1}=\bar{p}_{\varepsilon}(x+1 / k, t) \leq \frac{m}{m-1} \varepsilon^{m-1}+\frac{q}{k}+\frac{X}{2 k^{2}}+o\left(1 / k^{2}\right)
$$

for all sufficiently large $k \geq 1$. Due to the lower bound in (98) we infer by adding both inequalities that $0 \leq X / k^{2}+o\left(1 / k^{2}\right)$ for all sufficiently large $k \geq 1$. From this we deduce that $X \geq 0$. Using once more (89) as well as that $\bar{p}_{\varepsilon}$ satisfies the lateral boundary condition (29) pointwise it also holds

$$
\bar{p}_{\varepsilon}(x, t-1 / k) \leq \frac{m}{m-1} \varepsilon^{m-1}-\frac{a}{k}+o(1 / k)
$$

for all sufficiently large $k \geq 1$. Hence, by another application of the lower bound in (98) we infer that $0 \leq-a / k+o(1 / k)$ for all sufficiently large $k \geq 1$. In other words, it holds $a \leq 0$. To summarize we have shown that

$$
a-F\left(\bar{p}_{\varepsilon}(x, t), q, X\right)=a-(m-1) \bar{p}_{\varepsilon}(x, t) X-|q|^{2} \leq 0
$$

is satisfied for all $(x, t) \in \bigcup_{t \in\left(0, T^{*}\right)}\left(\nu B_{t}^{\varepsilon}+\partial I\right) \times\{t\}$ and all $(q, a, X) \in \mathcal{J}^{2,+} \bar{p}_{\varepsilon}(x, t)$. This proves (92) and thus concludes the proof of Lemma 15.

\subsection{Proof of Proposition 16 (Perron's solution for the limit problem)}

First note that $0 \leq \bar{p}_{\text {semi-rel }}(x, t)<\infty$ is satisfied for all $(x, t) \in \mathbf{R} \times\left[0, T^{*}\right)$ as a consequence of (99) and (98). Since $\bar{p}_{\varepsilon}(x, 0)=\bar{p}_{0, \varepsilon}(x)=\frac{m}{m-1}\left(u_{0}(x)+\varepsilon\right)^{m-1}$ for all $x \in \mathbf{R}$ we deduce that $\bar{p}_{\text {semi-rel }}(x)=\frac{m}{m-1} u_{0}(x)^{m-1}=\bar{p}_{0}(x)$ holds true for all $x \in \mathbf{R}$ since $u_{0}$ is continuous and the semi-relaxed limit (99) is defined in terms of backwards parabolic cylinders. 
We aim to show that $\bar{p}_{\text {semi-rel }}$ satisfies (95) for all $(x, t) \in \mathbf{R} \times\left(0, T^{*}\right)$ and all $(q, a, X) \in \mathcal{J}^{2,+} \bar{p}(x, t)$. For this, we will proceed in two steps as follows. Consider the lower-semicontinuous functional

$$
\begin{aligned}
& G_{\varepsilon, 1 \mathrm{sc}}^{-}: \mathbf{R} \times\left(0, T^{*}\right) \times[0, \infty) \times \mathbf{R}^{3} \rightarrow \mathbf{R} \\
& \quad(x, t, r, q, a, X) \mapsto \begin{cases}a-F(r, q, X), & (x, t) \in \bigcup_{t \in\left(0, T^{*}\right)}\left(v B_{t}^{\varepsilon}+I\right) \times\{t\}, \\
(a-F(r, q, X)) \wedge C_{\varepsilon}(r), & (x, t) \in \bigcup_{t \in\left(0, T^{*}\right)}\left(\nu B_{t}^{\varepsilon}+\partial I\right) \times\{t\}, \\
C_{\varepsilon}(r), & \text { else. }\end{cases}
\end{aligned}
$$

Since $\bar{p}_{\varepsilon}$ is a subsolution of (27)-(29) in the sense of Definition 14, it of course also satisfies

$$
G_{\varepsilon, 1 \mathrm{lsc}}^{-}\left(x, t, \bar{p}_{\varepsilon}(x, t), q, a, X\right) \leq 0
$$

for all $(x, t) \in \mathbf{R} \times\left(0, T^{*}\right)$ and all $(q, a, X) \in \mathcal{J}^{2,+} \bar{p}_{\varepsilon}(x, t)$. Now, define the lower semi-relaxed limit functional

$$
\begin{aligned}
& G_{\text {semi-rel }}^{-}(x, t, r, q, a, X) \\
& \quad:=\lim _{\varepsilon \rightarrow 0} \inf _{\hat{\varepsilon} \leq \varepsilon}\left\{G_{\hat{\varepsilon}, 1 \mathrm{sc}}^{-}(y, s, \hat{r}, \hat{q}, \hat{a}, \hat{X}):(y, s) \in \mathbf{R} \times\left(0, T^{*}\right) \times[0, \infty) \times \mathbf{R}^{3},\right. \\
& \left.\quad(y, s) \in(x-\varepsilon, x+\varepsilon) \times\left(t-\varepsilon^{2}, t\right],|(r-\hat{r}, q-\hat{q}, a-\hat{a}, X-\hat{X})|<\varepsilon\right\} .
\end{aligned}
$$

In a first step, we check that $\bar{p}_{\text {semi-rel }}$ satisfies

$$
G_{\text {semi-rel }}^{-}\left(x, t, \bar{p}_{\text {semi-rel }}(x, t), q, a, X\right) \leq 0
$$

for all $(x, t) \in \mathbf{R} \times\left(0, T^{*}\right)$ and all $(q, a, X) \in \mathcal{J}^{2,+} \bar{p}_{\text {semi-rel }}(x, t)$. In a second step, we identify the lower semi-relaxed limit $G_{\text {semi-rel }}^{-}$with the functional $G^{-}$defined in (93) as a consequence of the uniform convergence (9).

The validity of (107) is a consequence of standard viscosity theory. More precisely, (107) follows as a combination of [6, Lemma 6.1], [6, Remark 6.3] (for the purpose of subsolutions, the equations do not have to be proper) as well as the applicability of [6, Proposition 4.3]. Hence, let us show that $G_{\text {semi-rel }}^{-}=G^{-}$.

Due to the uniform convergence (8) and the definitions (93), (104) and (106) the statement is clear for space-time points $(x, t) \notin \bigcup_{t \in\left(0, T^{*}\right)}\left(\nu B_{t}+\partial I\right) \times\{t\}$. Hence, let us fix a point $(x, t) \in \bigcup_{t \in\left(0, T^{*}\right)}\left(\nu B_{t}+\partial I\right) \times\{t\}$ and an $\varepsilon>0$. Let

$$
\hat{\varepsilon}_{*}(\varepsilon):=\sup \left\{0<\hat{\varepsilon} \leq \varepsilon: \nu\left|B_{t}^{\hat{\varepsilon}}-B_{t}\right|<\varepsilon / 2\right\} .
$$

Note that $\hat{\varepsilon}_{*}>0$ because of the uniform convergence (8). We then have 


$$
\begin{aligned}
\lim _{\varepsilon \rightarrow 0} \inf _{(\hat{r}, \hat{q}, \hat{a}, \hat{X})}(\hat{a}-F(\hat{r}, \hat{q}, \hat{X})) \wedge C_{\varepsilon}(\hat{r}) & \leq G_{\text {semi-rel }}^{-}(x, t, r, q, a, X) \\
& \leq \lim _{\varepsilon \rightarrow 0} \inf _{(\hat{r}, \hat{q}, \hat{a}, \hat{X})}(\hat{a}-F(\hat{r}, \hat{q}, \hat{X})) \wedge C_{\hat{\varepsilon}_{*}(\varepsilon)}(\hat{r}),
\end{aligned}
$$

and where both inner infima run over all points $(\hat{r}, \hat{q}, \hat{a}, \hat{X}) \in[0, \infty) \times \mathbf{R}^{3}$ such that $|(r-\hat{r}, q-\hat{q}, a-\hat{a}, X-\hat{X})|<\varepsilon$. It follows that $G_{\text {semi-rel }}^{-}(x, t)=G^{-}(x, t)$ as claimed.

We have shown so far that $\bar{p}_{\text {semi-rel }}$ satisfies all conditions of Definition 14 except of being upper-semicontinuous on $\mathbf{R} \times\left[0, T^{*}\right)$. Since $\bar{p}_{\text {semi-rel }}$ is subject to (95), it is a classical fact of viscosity theory that also its upper-semicontinuous envelope satisfies (95). (A rigorous argument consists of applying [6, Lemma 6.1] to a constant sequence.) It remains to check whether the upper-semicontinuous envelope of $\bar{p}_{\text {semi-rel }}$ satisfies the initial condition with respect to $\bar{p}_{0}:=\frac{m}{m-1} u_{0}^{m-1}$. This follows from the following reasoning.

Consider the classical solution (and therefore also viscosity solution in the sense of [5, Definition 4]) $p_{\mathrm{visc}}^{\varepsilon}$ of (90) and (91) with initial pressure $p_{0, \varepsilon}:=\frac{m}{m-1}\left(u_{0}+\varepsilon\right)^{m-1}$. Because of (8), we can choose almost surely a space-time cylinder $Q=Q\left(B^{\varepsilon}\right)$ such that the parabolic closure of $\bigcup_{t \in\left(0, T^{*}\right)}\left(\nu B_{t}^{\varepsilon}+I\right) \times\{t\}$ is contained in $Q$. By the maximum principle, $p_{\mathrm{visc}}^{\varepsilon}$ is also subject to (98) on $\mathbf{R} \times\left[0, T^{*}\right)$. In particular, $p_{\mathrm{visc}}^{\varepsilon}$ dominates $\bar{p}_{\varepsilon}$ on the parabolic boundary of $Q$. Since $\bar{p}_{\varepsilon}$ is continuous and a subsolution of (90)-(91) by Lemma 15, it follows from [20, Lemma 2.5] that

$$
\bar{p}_{\varepsilon}(x, t) \leq p_{\mathrm{visc}}^{\varepsilon}(x, t)
$$

for all $(x, t)$ in the parabolic closure of $Q$, hence for all $(x, t) \in \mathbf{R} \times\left[0, T^{*}\right)$ by expanding $Q$ to $\mathbf{R} \times\left[0, T^{*}\right)$. Since $p_{\text {visc }}^{\varepsilon} \searrow p_{\text {visc }}$ with $p_{\text {visc }} \in C\left(\mathbf{R} \times\left[0, T^{*}\right)\right)$ being the viscosity solution of (90)-(91) with initial pressure $p_{0}(x):=\frac{m}{m-1} u_{0}(x)^{m-1}$ in the sense of [5, Definition 4], cf. the proof of [5, Lemma 2.2], we obtain together with (108) and an application of Dini's theorem the bound

$$
\begin{aligned}
\bar{p}_{\text {semi-rel }}(x, t) \leq & \lim _{\varepsilon \rightarrow 0} \sup _{\hat{\varepsilon} \leq \varepsilon}\left\{p_{\text {visc }}^{\hat{\varepsilon}}(y, s):(y, s) \in(x-\varepsilon, x+\varepsilon) \times\left(t-\varepsilon^{2}, t\right]\right\} \\
\leq & \lim _{\varepsilon \rightarrow 0} \sup \left\{p_{\text {visc }}^{\varepsilon}(y, s):(y, s) \in(x-\varepsilon, x+\varepsilon) \times\left(t-\varepsilon^{2}, t\right]\right\} \\
\leq & \lim _{\varepsilon \rightarrow 0} p_{\text {visc }}^{\varepsilon}(x, t) \\
& +\lim _{\varepsilon \rightarrow 0} \sup \left\{\left|p_{\text {visc }}^{\varepsilon}(y, s)-p_{\text {visc }}^{\varepsilon}(x, t)\right|:(y, s) \in(x-\varepsilon, x+\varepsilon) \times\left(t-\varepsilon^{2}, t\right]\right\} \\
\leq & p_{\text {visc }}(x, t)
\end{aligned}
$$

for all $(x, t) \in \mathbf{R} \times\left[0, T^{*}\right)$. However, since we already proved that $\bar{p}_{\text {semi-rel }}(x, 0)=$ $\frac{m}{m-1} u_{0}(x)^{m-1}=p_{\text {visc }}(x, 0)$ and $p_{\text {visc }}$ is continuous, it follows that also the uppersemicontinuous envelope of $\bar{p}_{\text {semi-rel }}$ attains the initial condition. Hence, it is a subsolution of (24)-(26) in the sense of Definition 14. 
We continue with the verification of the claims regarding $\bar{p}_{\max }$. To this end, consider first an arbitrary subsolution $\bar{p}$ of (24)-(26) with initial pressure $\bar{p}_{0}:=\frac{m}{m-1} u_{0}^{m-1}$ in the sense of Definition 14. The argument showing (109) more generally proves that

$$
\bar{p}(x, t) \leq p_{\text {visc }}(x, t)
$$

is satisfied for all $(x, t) \in \mathbf{R} \times\left[0, T^{*}\right)$. Hence, since we already established the existence of $a$ subsolution to (24)-(26) the definition of $\bar{p}_{\max }$ is meaningful, and the asserted bound (101) then follows at once from (110). In particular, $\bar{p}_{\max }<\infty$ is satisfied on $\mathbf{R} \times\left[0, T^{*}\right)$. That $\bar{p}_{\max }$ is subject to (95) is once again a classical fact from viscosity theory, see [6, Lemma 4.2]. Moreover, as we have already argued for the semi-relaxed limit $p_{\text {semi-rel }}$, it then follows that the upper-semicontinuous envelope of $\bar{p}_{\max }$ constitutes a subsolution of (24)-(26). However, by the definition of $\bar{p}_{\max }$ we may then infer that $\bar{p}_{\max }$ is actually equal to its upper-semicontinuous envelope; in particular, a subsolution of (24)-(26) in the sense of Definition 14. This concludes the proof of Proposition 16.

5.4 Proof of Proposition 17 (Comparison of transformed weak solution with viscosity solution)

It obviously suffices to prove that for all $t \in\left(0, T^{*}\right)$

$$
p(\cdot, t) \leq \bar{p}_{\text {semi-rel }}(\cdot, t)
$$

almost surely almost everywhere in $\mathbf{R}$. By definition of $p$, see (102), and since we have $\bar{p}_{\text {semi-rel }} \geq 0$ it moreover suffices to show that (111) is almost surely satisfied for all $t \in\left(0, T^{*}\right)$ almost everywhere in $v B_{t}+I$.

We aim to exploit Corollary 6, i.e., that the unique weak solution $u$ of (1)-(3) can be recovered by means of the Wong-Zakai approximations $u_{\varepsilon}$ from Lemma 4 , at least after employing an additional time-dependent shift (22). So fix an integer $M \geq 1$ as well as some $\kappa>0$. Let $\mathcal{C}_{\alpha}$ be the square integrable random variable of the estimate (10). Let finally $\varepsilon>0$ be fixed, and denote by $\varepsilon^{\prime}=\varepsilon^{\prime}(M, \varepsilon)$ the constant from (11).

We then have by means of the estimate (11) that

$$
\begin{aligned}
& \frac{m}{m-1} u_{\hat{\varepsilon}}\left(x-v B_{t}, t\right)^{m-1} \\
& \quad=\frac{m}{m-1} u_{\hat{\varepsilon}}\left(\left(x-v\left(B_{t}-B_{t}^{\hat{\varepsilon}}\right)\right)-v B_{t}^{\hat{\varepsilon}}, t\right)^{m-1} \\
& \quad \leq \sup _{\hat{\varepsilon} \leq \varepsilon}\left\{\bar{p}_{\hat{\varepsilon}}(y, s):(y, s) \in \mathbf{R} \times\left[0, T^{*}\right),(y, s) \in(x-\varepsilon, x+\varepsilon) \times\left(t-\varepsilon^{2}, t\right]\right\} \\
& \quad=: \bar{P}_{\varepsilon}(x, t)
\end{aligned}
$$

almost surely on $\left\{\mathcal{C}_{\alpha} \leq M\right\}$ for all $\hat{\varepsilon} \leq \varepsilon \wedge \varepsilon^{\prime}$ and all $(x, t) \in \bigcup_{t \in\left(0, T^{*}\right)}\left(\nu B_{t}+I\right) \times\{t\}$. Fix $t \in\left(0, T^{*}\right)$, let $\phi \in C_{\mathrm{cpt}}^{\infty}\left(\nu B_{t}+I ;[0, \infty)\right)$ be an arbitrary test function and let $A \in \mathcal{F}_{T^{*}}$. It follows from (112) that 


$$
\begin{aligned}
0 & \leq \mathbf{E} \chi_{A \cap\left\{C_{\alpha} \leq M\right\}} \int_{\mathbf{R}} \phi\left(x+v B_{t}^{\hat{\varepsilon}}\right) \frac{m}{m-1} u_{\hat{\varepsilon}}^{\leftarrow}(x, t)^{m-1} \mathrm{~d} x \\
& \leq \mathbf{E} \chi_{A \cap\left\{C_{\alpha} \leq M\right\}} \int_{\mathbf{R}} \phi(x) \bar{P}_{\varepsilon}(x, t) \mathrm{d} x
\end{aligned}
$$

for all $\hat{\varepsilon} \leq \varepsilon \wedge \varepsilon^{\prime}$, all $t \in\left(0, T^{*}\right)$, all $\phi \in C_{\mathrm{cpt}}^{\infty}\left(\nu B_{t}+I ;[0, \infty)\right)$ and all $A \in \mathcal{F}_{T^{*}}$. As a consequence of the bound (113), the convergences in (9) resp. Corollary 6 as well as the definition (99) of the semi-relaxed limit $\bar{p}_{\text {semi-rel, }}$, we thus obtain the estimate

$$
\begin{aligned}
0 & \leq \mathbf{E} \chi_{A \cap\left\{C_{\alpha} \leq M\right\}} \int_{\mathbf{R}} \phi(x) \frac{m}{m-1} u\left(x-v B_{t}, t\right)^{m-1} \mathrm{~d} x \\
& \leq \mathbf{E} \chi_{A \cap\left\{C_{\alpha} \leq M\right\}} \int_{\mathbf{R}} \phi(x) \bar{p}_{\text {semi-rel }}(x, t) \mathrm{d} x
\end{aligned}
$$

for all $t \in\left(0, T^{*}\right)$, all $\phi \in C_{\mathrm{cpt}}^{\infty}\left(\nu B_{t}+I ;[0, \infty)\right)$ and all $A \in \mathcal{F}_{T^{*}}$. Since the test function and $A$ are arbitrary, we deduce that the following bound holds true for all $t \in\left(0, T^{*}\right)$ almost surely on $\left\{C_{\alpha} \leq M\right\}$ almost everywhere in $v B_{t}+I$ :

$$
p(\cdot, t)=\frac{m}{m-1} u\left(\cdot-v B_{t}, t\right)^{m-1} \leq \bar{p}_{\text {semi-rel }}(\cdot, t) .
$$

Since $M \geq 1$ is arbitrary, and the random variable $\mathcal{C}_{\alpha}$ is integrable, we may infer from this the desired bound (103). This concludes the proof of Proposition 17.

5.5 Proof of Theorem 3 (Finite time extinction with probability one)

Let $p_{\text {visc }} \in C(\mathbf{R} \times[0, \infty))$ be the unique viscosity solution of (90)-(91) with initial pressure $p_{0}(x):=\frac{m}{m-1} u_{0}(x)^{m-1}$ in the sense of [5, Definition 4]. Since the time horizon $T^{*}<\infty$ was arbitrary in Proposition 16 and Proposition 17, we have constructed almost surely a (maximal) subsolution $\bar{p}_{\max }$ of (24)-(26) on $\mathbf{R} \times[0, \infty)$ in the sense of Definition 14 such that it holds almost surely

$$
\bar{p}_{\text {max }}(x, t) \leq p_{\text {visc }}(x, t)
$$

for all $(x, t) \in \mathbf{R} \times[0, \infty)$, as well as for all $t \in(0, \infty)$

$$
p(x, t) \leq \bar{p}_{\max }(x, t)
$$

almost surely almost everywhere in $\mathbf{R}$, where $p$ is defined on $\mathbf{R} \times(0, \infty)$ via (102) based on the unique weak solution of the Cauchy-Dirichlet problem (1)-(3) on $\mathbf{R} \times(0, \infty)$ with initial density $u_{0}$.

Next, we choose a delayed Barenblatt solution $\mathcal{B}$ (written in terms of the pressure variable) with free boundary $\partial I$ at $t=0$ and which strictly dominates the initial density, i.e., $\{\mathcal{B}(\cdot, 0)=0\}=\partial I$ and $u_{0}<\mathcal{B}(\cdot, 0)$ on $I$. The free boundary associated to the Barenblatt solution $\mathcal{B}$ can then be written as $\bigcup_{t \in[0, \infty)}\left(\bar{M} t^{\frac{1}{m+1}}+\partial I\right) \times\{t\}$ for a constant $\bar{M}=\bar{M}\left(u_{0}\right)>0$ depending only on the initial density. 
We remark that the Barenblatt solution $\mathcal{B}$ is a viscosity solution of (90) and (91) in the sense of [5, Definition 4] strictly dominating $p_{\text {visc }}$ at $t=0$ in the sense of [5, Definition 5]. Hence, it follows from the comparison principle [5, Theorem 4.1] that almost surely

$$
p_{\text {visc }}(x, t) \leq \mathcal{B}(x, t)
$$

for all $(x, t) \in \mathbf{R} \times[0, \infty)$.

Let $M>\bar{M}$ be fixed but otherwise arbitrary, and define the stopping time $\hat{T}_{\text {extinct }}:=$ $\inf \left\{t \geq 0:\left|B_{t}\right| \geq \mathcal{L}^{1}(I)+M t^{\frac{1}{m+1}}\right\}$ as in the statement of Theorem 3. Furthermore, fix $T \in(0, \infty)$ and consider $\hat{\mathcal{A}}_{T}:=\left\{\hat{T}_{\text {extinct }} \leq T\right\}$. Note that $\hat{T}_{\text {extinct }}>0$. By almost sure continuity of $B_{t}$ and since $M>\bar{M}$, there is almost surely on $\hat{\mathcal{A}}_{T}$ some $t_{q} \in \mathbb{Q} \cap(0, T]$ such that $\left|B_{t_{q}}\right| \geq \mathcal{L}^{1}(I)+\bar{M} t_{q}^{\frac{1}{m+1}}$. As a consequence of (115) and the choice of $\bar{M}$, we deduce that almost surely on $\hat{\mathcal{A}}_{T}$ it holds $\bar{p}_{\text {max }}\left(\cdot, t_{q}\right) \equiv 0$. Moreover, (116) entails that almost surely we have $p \leq p_{\max }$ for all $t \in \mathbb{Q} \cap(0, \infty)$ almost everywhere in $\mathbf{R}$. Hence, we may deduce that $\overline{\mathcal{A}}_{T} \subset\left\{T_{\text {extinct }} \leq T\right\}$ up to some $\mathbf{P}$ null set. This proves the desired bound (6).

Finite time extinction with probability one now follows from standard properties of Brownian motion. Indeed, assume that we have $\mathbf{P}\left(\hat{T}_{\text {extinct }}<\infty\right)<1$. There would then exist a constant $C<\infty$ such that on a set with non-vanishing probability it holds $0 \leq t^{-\frac{1}{2}}\left|B_{t}\right|<C$ for all $t \in(1, \infty)$; a contradiction. Hence, $\mathbf{P}\left(\hat{T}_{\text {extinct }}<\infty\right)=1$ which also by (6) entails that $\mathbf{P}\left(T_{\text {extinct }}<\infty\right)=1$.

It remains to prove that $u(\cdot, T)$ vanishes almost surely almost everywhere in $I$ from the stopping time $\hat{T}_{\text {extinct }}$ onwards. So let $T \in(0, \infty)$ be fixed. We infer from (115) together with $(117)$ that almost surely $\bar{p}_{\text {max }}\left(\cdot, \hat{T}_{\text {extinct }}\right) \equiv 0$. In addition, we note that $\bar{p}_{\text {max }}$ is almost surely a subsolution of (90) and (91) on $\mathbf{R} \times[0, \infty)$ with initial pressure $p_{0}(x):=\frac{m}{m-1} u_{0}(x)^{m-1}$ in the sense of Definition 13. For a proof, the only non-trivial case is that of a lateral boundary point $(x, t) \in \bigcup_{t \in(0, \infty)}\left(v B_{t}+I\right) \times\{t\}$ such that $C\left(\bar{p}_{\max }(x, t)\right)=\bar{p}_{\max }(x, t) \leq 0$. Since $\bar{p}_{\max }(x, t) \geq 0$ by construction, it follows that $\bar{p}_{\text {max }}(x, t)=0$. From this point onwards one may argue as for $\bar{p}_{\varepsilon}$ in the proof of Lemma 15.

However, once a subsolution became trivial it stays trivial (by comparison with the trivial viscosity solution). We deduce that $\bar{p}_{\max }(\cdot, T) \equiv 0$ is satisfied almost surely on $\left\{T \geq \hat{T}_{\text {extinct }}\right\}$. The claim thus follows from another application of (116). This concludes the proof of Theorem 3 .

Open Access This article is licensed under a Creative Commons Attribution 4.0 International License, which permits use, sharing, adaptation, distribution and reproduction in any medium or format, as long as you give appropriate credit to the original author(s) and the source, provide a link to the Creative Commons licence, and indicate if changes were made. The images or other third party material in this article are included in the article's Creative Commons licence, unless indicated otherwise in a credit line to the material. If material is not included in the article's Creative Commons licence and your intended use is not permitted by statutory regulation or exceeds the permitted use, you will need to obtain permission directly from the copyright holder. To view a copy of this licence, visit http://creativecommons.org/licenses/by/4.0/. 


\section{References}

1. Andreianov, B.P., Igbida, N.: Uniqueness for inhomogeneous Dirichlet problem for elliptic-parabolic equations. Proc. R. Soc. Edinburgh Sect. A 137, 1119-1133 (2007). https://doi.org/10.1017/ S0308210505001290

2. Barbu, V., Da Prato, G., Röckner, M.: Finite time extinction for solutions to fast diffusion stochastic porous media equations. C. R. Acad. Sci. Paris Ser. I I(347), 81-84 (2009). https://doi.org/10.1016/j. crma.2008.11.018

3. Barbu, V., Da Prato, G., Röckner, M.: Stochastic porous media equations and self-organized criticality. Commun. Math. Phys. 285, 901-923 (2009). https://doi.org/10.1007/s00220-008-0651-X

4. Barbu, V., Da Prato, G., Röckner, M.: Finite time extinction of solutions to fast diffusion equations driven by linear multiplicative noise. J. Math. Anal. Appl. 389, 147-164 (2012). https://doi.org/10. 1016/j.jmaa.2011.11.045

5. Caffarelli, L., Vázquez, J.L.: Viscosity solutions for the porous medium equation. In: Proceedings of symposia in pure mathematics. Am. Math. Soc. Provid. 65, 13-26 (1999). https://doi.org/10.1090/ pspum/065/1662747

6. Crandall, M.G., Ishii, H., Lions, P.-L.: User's guide to viscosity solutions of second order partial differential equations. Bull. Am. Math. Soc. 27, 1-67 (1992). https://doi.org/10.1090/s0273-09791992-00266-5

7. Dareiotis, K., Gerencsér, M., Gess, B.: Entropy solutions for stochastic porous media equations. J. Differ. Equ. 266, 3732-3763 (2019). https://doi.org/10.1016/j.jde.2018.09.012

8. Dareiotis, K., Gess, B.: Supremum estimates for degenerate, quasilinear stochastic partial differential equations. Ann. Inst. Henri Poincaré Probab. Stat. 55, 1765-1796 (2019). https://doi.org/10.1214/18AIHP934

9. Dareiotis, K., Gess, B.: Nonlinear diffusion equations with nonlinear gradient noise. Electron. J. Probab. 25, 1-43 (2020). https://doi.org/10.1214/20-EJP436

10. Dareiotis, K., Gess, B., Tsatsoulis, P.: Ergodicity for stochastic porous media equations. SIAM J. Math. Anal. 52, 4524-4564 (2020). https://doi.org/10.1137/19M1278521

11. Fehrman, B., Gess, B.: Well-posedness of nonlinear diffusion equations with nonlinear, conservative noise. Arch. Ration. Mech. Anal. 233, 249-322 (2019). https://doi.org/10.1007/s00205-019-01357W

12. Gerencsér, M.: Boundary regularity of stochastic PDEs. Ann. Probab. 47, 804-834 (2019). https://doi. org/10.1214/18-AOP1272

13. Gess, B.: Finite time extinction for stochastic sign fast diffusion and self-organized criticality. Commun. Math. Phys. 335, 2355-2383 (2015). https://doi.org/10.1007/s00220-014-2225-4

14. Kružkov, S.N.: First order quasilinear equations in several independent variables. Math. USSR-Sb 10, 217-243 (1970). https://doi.org/10.1070/SM1970v010n02ABEH002156

15. Krylov, N.V.: Brownian trajectory is a regular lateral boundary for the heat equation. SIAM J. Math. Anal. 34, 1167-1182 (2003). https://doi.org/10.1137/S0036141002402980

16. Krylov, N.V.: One more square root law for Brownian motion and its application to SPDEs. Probab. Theory Relat. Fields 127, 496-512 (2003). https://doi.org/10.1007/s00440-003-0301-3

17. Ladyzhenskaya, O.A., Solonnikov, V.A., Ural'tseva, N.N.: Linear and Quasilinear Equations of Parabolic Type. Translations of Mathematical Monographs, vol. 23. American Mathematical Society, Providence (1968)

18. Turra, M.: Existence and extinction in finite time for Stratonovich gradient noise porous media equations. Evol. Equ. Control Theory 8, 867-882 (2019). https://doi.org/10.3934/eect.2019042

19. Vázquez, J.L.: The Porous Medium Equation: Mathematical Theory. Clarendon Press, Oxford Mathematical Monographs, Oxford (2007)

20. Vázquez, J.L., Brändle, C.: Viscosity solutions for quasilinear degenerate parabolic equations of porous medium type. Indiana Univ. Math. J. 54, 817-860 (2005). https://doi.org/10.1512/iumj.2005.54.2565

Publisher's Note Springer Nature remains neutral with regard to jurisdictional claims in published maps and institutional affiliations. 